\title{
Quantitative Regularity for the Navier-Stokes Equations Via Spatial Concentration
}

\section{Tobias Barker $^{1}$, Christophe Prange ${ }^{2}$}

1 Mathematics Institute, University of Warwick, Coventry, UK. E-mail: tobiasbarker5@gmail.com

2 Laboratoire de Mathématiques AGM, UMR CNRS 8088, Cergy Paris Université, Cergy-Pontoise, France. E-mail: christophe.prange@cyu.fr

Abstract: This paper is concerned with quantitative estimates for the Navier-Stokes equations. First we investigate the relation of quantitative bounds to the behavior of critical norms near a potential singularity with Type I bound $\|u\|_{L_{t}^{\infty}} L_{x}^{3, \infty} \leq M$. Namely, we show that if $T^{*}$ is a first blow-up time and $\left(0, T^{*}\right)$ is a singular point then

$$
\|u(\cdot, t)\|_{L^{3}\left(B_{0}(R)\right)} \geq C(M) \log \left(\frac{1}{T^{*}-t}\right), \quad R=O\left(\left(T^{*}-t\right)^{\frac{1}{2}-}\right) .
$$

We demonstrate that this potential blow-up rate is optimal for a certain class of potential non-zero backward discretely self-similar solutions. Second, we quantify the result of Seregin (Commun Math Phys 312(3):833-845, 2012), which says that if $u$ is a smooth finite-energy solution to the Navier-Stokes equations on $\mathbb{R}^{3} \times(0,1)$ with

$$
\sup _{n}\left\|u\left(\cdot, t_{(n)}\right)\right\|_{L^{3}\left(\mathbb{R}^{3}\right)}<\infty \text { and } t_{(n)} \uparrow 1,
$$

then $u$ does not blow-up at $t=1$. To prove our results we develop a new strategy for proving quantitative bounds for the Navier-Stokes equations. This hinges on local-inspace smoothing results (near the initial time) established by Jia and Šverák (2014), together with quantitative arguments using Carleman inequalities given by Tao (2019). Moreover, the technology developed here enables us in particular to give a quantitative bound for the number of singular points in a Type I blow-up scenario.

\section{Introduction}

In this paper, we consider the three-dimensional incompressible Navier-Stokes equations

$$
\partial_{t} u-\Delta u+u \cdot \nabla u+\nabla p=0, \quad \nabla \cdot u=0
$$


on $\mathbb{R}^{3} \times(0, T)$ where $T \in(0, \infty)$, supplemented with an initial condition $u(\cdot, 0)=$ $u_{0}(x)$. It is well known that this system of equations is invariant with respect to the following rescaling

$$
\left(u_{\lambda}(x, t), p_{\lambda}(x, t), u_{0 \lambda}(x)\right):=\left(\lambda u\left(\lambda x, \lambda^{2} t\right), \lambda^{2} p\left(\lambda x, \lambda^{2} t\right), \lambda u_{0}(\lambda x)\right), \quad \lambda>0 .
$$

The question as to whether or not finite-energy solutions ${ }^{1}$, with divergence-free Schwartz class initial data, remain smooth for all times is a Millennium Prize problem [14]. The first necessary conditions for such a solution to lose smoothness or to 'blow-up' at time $T^{*}>0^{2}$ were given in the seminal paper of Leray [27]. In particular, in [27] it is shown that if $T^{*}$ is a first blow-up time of $u$ then we necessarily have

$$
\|u(\cdot, t)\|_{L^{p}\left(\mathbb{R}^{3}\right)} \geq \frac{C(p)}{\left(T^{*}-t\right)^{\frac{1}{2}\left(1-\frac{3}{p}\right)}}, \quad \text { for } \quad p \in(3, \infty] .
$$

The $L^{3}\left(\mathbb{R}^{3}\right)$ norm is scale-invariant or 'critical'3 with respect to the Navier-Stokes rescaling. Its role in the regularity theory of the Navier-Stokes equations is much more subtle than that of the subcritical $L^{p}\left(\mathbb{R}^{3}\right)$ norms with $3<p \leq \infty$. In particular, it is demonstrated by an elementary scaling argument in $[4]^{4}$ that if the set of finite-energy solutions to the Navier-Stokes equations (with Schwartz class initial data) that blows-up is non-empty, there cannot exist an universal function $f:(0, \infty) \rightarrow(0, \infty)$ such that the following analogue of (3) holds true:

$$
\lim _{s \rightarrow 0^{+}} f(s)=\infty,
$$

and for all $T^{*}>0$, if $u$ is a finite-energy solution to the Navier-Stokes equations (with Schwartz class initial data) that first blows-up at $T^{*}>0$ then $u$ necessarily satisfies

$$
\|u(\cdot, t)\|_{L^{3}\left(\mathbb{R}^{3}\right)} \geq f\left(T^{*}-t\right)
$$

for all $t \in\left[0, T^{*}\right)$.

In the celebrated paper [13] of Escauriaza, Seregin and Šverák, it was shown that if a finite-energy solution $u$ first blows-up at $T^{*}>0$ then necessarily

$$
\underset{t \uparrow T^{*}}{\lim \sup }\|u(\cdot, t)\|_{L^{3}\left(\mathbb{R}^{3}\right)}=\infty .
$$

The proof in [13] is by contradiction. A rescaling procedure or 'zoom-in' is performed ${ }^{5}$ using (2) and a compactness argument is applied. This gives a non-zero limit solution to the Navier-Stokes equations that vanishes at the final moment in time. The contradiction is achieved by showing that the limit function must be zero by applying a Liouville type theorem based on backward uniqueness for parabolic operators satisfying certain differential inequalities. By now there are many generalizations of (6) to cases of other critical norms. See, for example, $[12,16,35,50]$.

\footnotetext{
1 See the definition of a finite-energy solution in Sect. 1.4 'Notations'.

2 See Sect. 1.4 for a definition.

3 See Sect. 1.4 for a definition.

4 The argument in [4] is in turn taken from the talk given by G. Seregin. 'A certain necessary condition of possible blow up for the Navier-Stokes equations'. APDE seminar, University of Sussex, 03 March 2014.

5 It is worth noting that [13] appears to be the first instance where arguments involving 'zooming in' and passage to a limit have been applied to the Navier-Stokes equations.
} 
Let us mention the arguments in [13] and the aforementioned works are by contradiction and hence are qualitative. It is worth noting that the result in [13], together with a proof by contradiction based on the 'persistence of singularities' lemma in [36] (specifically Lemma 2.2 in [36]), gives the following. Namely, that there exists an $F:(0, \infty) \rightarrow(0, \infty)$ such that if $u$ is a finite-energy solution to the Navier-Stokes equations then

$$
\|u\|_{L^{\infty}\left(0,1 ; L^{3}\left(\mathbb{R}^{3}\right)\right)}<\infty \Rightarrow\|u\|_{L^{\infty}\left(\mathbb{R}^{3} \times\left(\frac{1}{2}, 1\right)\right)} \leq F\left(\|u\|_{L^{\infty}\left(0,1 ; L^{3}\left(\mathbb{R}^{3}\right)\right)}\right) .
$$

Such an argument is obtained by a compactness method and gives no explicit ${ }^{6}$ information about $F$. In a remarkable recent development [47], Tao used a new approach to provide the first explicit quantitative estimates for solutions of the Navier-Stokes equations belonging to the critical space $L^{\infty}\left(0, T ; L^{3}\left(\mathbb{R}^{3}\right)\right)$. As a consequence of these quantitative estimates, Tao showed in [47] that if a finite-energy solution $u$ first blows-up at $T^{*}>0$ then for some absolute constant $c>0$

$$
\limsup _{t \uparrow T^{*}} \frac{\|u(\cdot, t)\|_{L^{3}\left(\mathbb{R}^{3}\right)}}{\left(\log \log \log \frac{1}{T^{*}-t}\right)^{c}}=\infty .
$$

Since there cannot exist $f$ such that (4)-(5) holds true, at first sight (8) may seem somewhat surprising, though it is not conflicting with such a fact. Notice that

$$
\frac{\|u(\cdot, t)\|_{L^{3}\left(\mathbb{R}^{3}\right)}}{\left(\log \log \log \frac{1}{T^{*}-t}\right)^{c}}
$$

is not invariant with respect to the Navier-Stokes scaling (2) but is slightly supercritical ${ }^{7}$ due to the presence of the logarithmic denominator. Let us also mention that prior to Tao's paper [47], in the presence of axial symmetry, a different slightly supercritical regularity criteria was obtained in [34].

The contribution of our present paper is to develop a new strategy for proving quantitative estimates (see Propositions 2.1 and 2.2) for the Navier-Stokes equations, which then enables us to build upon Tao's work [47] to quantify critical norms. Our first Theorem involves applying the backward propagation of concentration stated in Proposition 2.1 below to give a new necessary condition for solutions to the Navier-Stokes equations to possess a Type I blow-up. In the case of a Type I blow-up at $T^{*}$ the nonlinearity in (2) is heuristically balanced with the diffusion. Despite this, it remains a long standing open problem whether or not Type I blow-ups can be ruled out when $M$ is large. Let us now state our first theorem.

Theorem A. (rate of blow-up, Type I). There exists a universal constant $M_{0} \in[1, \infty)$ such that for all $M \geq M_{0}$ the following holds true.

Assume that $u$ is a mild solution to the Navier-Stokes equations on $\mathbb{R}^{3} \times\left[0, T^{*}\right)$ with $u \in L_{\text {loc }}^{\infty}\left(\left[0, T^{*}\right) ; L^{\infty}\left(\mathbb{R}^{3}\right)\right)$.

Assume in addition that $\left(0, T^{*}\right)$ is a Type I blow-up, i.e.

(1) $\|u\|_{L_{t}^{\infty} L_{x}^{3, \infty}\left(\mathbb{R}^{3} \times\left(0, T^{*}\right)\right)} \leq M,^{8}$

\footnotetext{
6 Throughout this paper we will sometimes use the terminology 'effective' bounds to describe an explicit quantitative bound. An abstract quantitative bound will sometimes be referred to as 'non-effective'.

7 See Sect. 1.4 for a definition.

8 Under these assumptions and by interpolation, we infer that $u$ is a 'smooth solution with sufficient decay' in the sense of the definition in Sect. 1.4. This enables us to satisfy the hypothesis needed in Sects. 5-7.
} 
(2) $u$ has a singular point at $(x, t)=\left(0, T^{*}\right)$. In particular $u \notin L_{x, t}^{\infty}\left(Q_{\left(0, T^{*}\right)}(r)\right)$ for all sufficiently small $r>0$.

Then the above assumptions imply that there exists $S_{B P}(M) \in\left(0, \frac{1}{4}\right]^{9}$ such that for any $t \in\left(\frac{T^{*}}{2}, T^{*}\right)$ and

$$
R \in\left(2 \sqrt{\frac{T^{*}-t}{S_{B P}(M)}}, e^{M^{1022}} \sqrt{T^{*}}\right)
$$

we have

$$
\int_{|x|<R}|u(x, t)|^{3} d x \geq \frac{\log \left(\frac{R^{2}}{M^{802}\left|T^{*}-t\right|}\right)}{\exp \left(\exp \left(M^{1025}\right)\right)} .
$$

This theorem is proved in Sect. 2.2 below. Notice that in Theorem A, not only is the rate new but also the fact that the $L^{3}$ norm blows up on a ball of radius $O\left(\left(T^{*}-t\right)^{\frac{1}{2}-}\right)$ around any Type I singularity. Previously in [28] (specifically Theorem 1.3 in [28]), it was shown that if a solution blows up (without Type I bound) then the $L^{3}$ norm blows up on certain non-explicit concentrating sets.

The Navier-Stokes scaling symmetry (2) plays a role in considering blow-up ansatzes having certain symmetry properties. In [27], Leray suggested the blow-up ansatz of backward self-similar solutions ${ }^{10}$, which are invariant with respect to the Navier-Stokes rescaling. Although the existence of non-zero backward self-similar solutions to the Navier-Stokes equations has been ruled out under general circumstances in [32] and [48], the existence of non-zero backward discretely self-similar solutions remains open. This was first stated as an open problem in [49]. Here we say that $u$ is a backward discretely self-similar solution ( $\lambda$-DSS) if there exists $\lambda \in(1, \infty)$ such that $u(x, t)=\lambda u\left(\lambda x, \lambda^{2} t\right)$ for all $(x, t) \in \mathbb{R}^{3} \times(-\infty, 0)$. As a corollary to Theorem A, we show that if there exists a non-zero $\lambda$-DSS (having certain decay properties which we will specify), then the localized blow-up rate (10) in Theorem A is optimal.

Corollary 1.1 Suppose $u: \mathbb{R}^{3} \times(-\infty, 0) \rightarrow \mathbb{R}^{3}$ is a non-zero $\lambda-D S S$ to the NavierStokes equations such that

$$
u \in C^{\infty}\left(\mathbb{R}^{3} \times(-\infty, 0)\right) \cap C\left((-\infty, 0) ; L^{p}\left(\mathbb{R}^{3}\right)\right),
$$

for some $p \in[3, \infty)$. There exists $M>1$ and $S_{B P}(M) \in\left(0, \frac{1}{4}\right]$ (see Theorem $A$ ) such that the following holds true. Namely, for all $t \in(-\infty, 0)$ and

$$
R \in\left(2 \sqrt{\frac{-t}{S_{B P}(M)}}, \infty\right)
$$

we have

$$
4 \pi M^{3} \log \frac{2 R^{2}}{|t|} \geq \int_{|x|<R}|u(x, t)|^{3} d x \geq \frac{\log \left(\frac{R^{2}}{M^{802}|t|}\right)}{\exp \left(\exp \left(M^{1025}\right)\right)} .
$$

\footnotetext{
${ }^{9}$ Note that $S_{B P}(M)=S^{*}(M)$ where $S^{*}(M)$ is defined in [6, Theorem 1]. Observing p.1520 of [6], one can infer that $S_{B P}(M) \sim M^{-30}$. Thus, the interval in (9) makes sense.

10 See Sect. 1.4 for a definition.
} 
This corollary is proved in Sect. 2.2 below.

In [13], it is shown that if $u$ is a finite-energy solution to the Navier-Stokes equations in $C^{\infty}\left(\mathbb{R}^{3} \times(0,1)\right)$, with Schwartz initial data, then

$$
\|u\|_{L^{\infty}\left((0,1) ; L^{3}\left(\mathbb{R}^{3}\right)\right)}<\infty
$$

implies that $u$ does not blow-up at time 1 (namely $u \in L_{t, l o c}^{\infty}\left((0,1] ; L^{\infty}\left(\mathbb{R}^{3}\right)\right)$ ). In [37], Seregin refined the assumption (13) to

$$
\sup _{n}\left\|u\left(\cdot, t_{(n)}\right)\right\|_{L^{3}\left(\mathbb{R}^{3}\right)}<\infty \text { with } t_{(n)} \uparrow 1 .
$$

Seregin's result implies that if $u$ is a finite-energy solution that first loses smoothness at $T^{*}>0$ then

$$
\lim _{t \uparrow T^{*}}\|u(\cdot, t)\|_{L^{3}\left(\mathbb{R}^{3}\right)}=\infty .
$$

This result has been further refined to other wider critical spaces and to domains other than $\mathbb{R}^{3}$. See, for example, [1-3,7,29]. All these arguments are qualitative and achieved by contradiction and compactness arguments. It is interesting to note that in contrast to (7) it is not known ${ }^{11}$, even abstractly, if there exists a $G:(0, \infty) \rightarrow(0, \infty)$ such that if $u$ is a finite-energy solution of the Navier-Stokes equations belonging to $C^{\infty}\left(\mathbb{R}^{3} \times(0,1]\right)$ then

$$
\sup _{n}\left\|u\left(\cdot, t_{(n)}\right)\right\|_{L^{3}\left(\mathbb{R}^{3}\right)}<\infty \text { with } t_{(n)} \uparrow 1 \Rightarrow\|u(\cdot, 1)\|_{L^{\infty}\left(\mathbb{R}^{3}\right)} \leq G\left(\sup _{n}\left\|u\left(\cdot, t_{(n)}\right)\right\|_{L^{3}\left(\mathbb{R}^{3}\right)}\right) .
$$

In our second main theorem, we apply Proposition 2.2 to fully quantify Seregin's result in [37], which generalizes Theorem 1.2 in [47]. Now let us state our second theorem.

Theorem B. (main quantitative estimate, time slices; quantification of Seregin's result) There exists a universal constant $M_{1} \in[1, \infty)$ such that the following holds true. Let $M \in\left[M_{1}, \infty\right)$. We define $M^{b}$ by ${ }^{12}$

$$
M^{b}:=\exp \left(\frac{L_{*} M^{5}}{2}\right),
$$

for an appropriate constant $L_{*} \in(0, \infty)$. Let $(u, p)$ be a finite-energy $C^{\infty}\left(\mathbb{R}^{3} \times(-1,0)\right)$ solution to the Navier-Stokes equations (1) on $\mathbb{R}^{3} \times[-1,0]^{13}$. Assume that there exists $t_{(k)} \in[-1,0)$ such that

$$
t_{(k)} \uparrow 0 \text { with } \sup _{k}\left\|u\left(\cdot, t_{(k)}\right)\right\|_{L^{3}\left(\mathbb{R}^{3}\right)} \leq M .
$$

\footnotetext{
11 Assume for contradiction that (16) does not hold. Then we have a sequence of solutions $\left(u^{k}\right)_{k \in \mathbb{N}}$ such that $\left\|u^{(k)}(\cdot, 1)\right\|_{L^{\infty}\left(\mathbb{R}^{3}\right)} \uparrow \infty$ and, for each $k$, a sequence of associated time slices $t_{(n)}^{k} \uparrow 1$ such that $\sup _{n, k}\left\|u^{(k)}\left(\cdot, t_{(n)}^{k}\right)\right\|_{L^{3}\left(\mathbb{R}^{3}\right)}=M<\infty$. The main block for the contradiction argument to go through is that the sequence $\left(t_{(n)}^{k}\right)_{n \in \mathbb{N}}$ may be different for distinct indices $k$.

12 In particular, $M^{b}$ is chosen such that the following is true. If $(u, p)$ is a suitable finite-energy solution (defined in Sect. 1.4 'Notations') to Navier-Stokes on $\mathbb{R}^{3} \times[0, T)$ with $L^{3}$ initial data $\left\|u_{0}\right\|_{L^{3}\left(\mathbb{R}^{3}\right)} \leq M$, then $w:=u-e^{t \Delta} u_{0}$ satisfies $\|w(\cdot, t)\|_{L^{2}\left(\mathbb{R}^{3}\right)}^{2}+\int_{0}^{t} \int_{\mathbb{R}^{3}}|\nabla w|^{2} d x d t^{\prime} \leq\left(M^{b}\right)^{4} t^{\frac{1}{2}}$ for $t \in(0, T)$ and $M$ larger than a universal constant. See Lemma 3.3.

13 Notice that smoothness is needed here to have the energy inequality starting from every time $t_{k}$, and not for almost every $t^{\prime} \in(-1,0)$, see (43) as would be the case if $(u, p)$ was just a suitable finite-energy solution.
} 
Select any "well-separated" subsequence (still denoted $\left.t_{(k)}\right)$ such that

$$
\sup _{k} \frac{-t_{(k+1)}}{-t_{(k)}}<\exp \left(-2\left(M^{b}\right)^{1223}\right) .
$$

Then for

we have the bound

$$
j:=\left\lceil\exp \left(\exp \left(\left(M^{b}\right)^{1224}\right)\right)\right\rceil+1
$$

$$
\|u\|_{L^{\infty}\left(\mathbb{R}^{3} \times\left(\frac{t_{(j+1)}}{4}, 0\right)\right)} \leq \frac{C_{1} M^{-23}}{\left(-t_{(j+1)}\right)^{\frac{1}{2}}},
$$

for a universal constant $C_{1} \in(0, \infty)$.

This theorem is proved in Sect. 2.2 below.

Further applications. Section 4 contains three further applications of the technology developed in the present paper: (i) Proposition 4.1, a regularity criteria based on an effective $^{14}$ relative smallness condition in the Type I setting, (ii) Corollary 4.3, an effective bound for the number of singular points in a Type I blow-up scenario, (iii) Proposition 4.4, a regularity criteria based on an effective relative smallness condition on the $L^{3}$ norm at initial and final time. Non-effective quantitative bounds of the above results were previously obtained by compactness methods: for (ii) see [12, Theorem 2], for (iii) see [2, Theorem 4.1 (i)].

1.1. Comparison to previous literature and novelty of our results. Theorems A and B in this paper follow from new quantitative estimates for the Navier-Stokes equations (Propositions 2.1 and 2.2), which build upon recent breakthrough work by Tao in [47]. In particular, Tao shows that for classical $^{15}$ solutions to the Navier-Stokes equations

$$
\|u\|_{L_{t}^{\infty} L_{x}^{3}\left(\mathbb{R}^{3} \times(0,1)\right)} \leq A \Rightarrow\|u(\cdot, t)\|_{L^{\infty}\left(\mathbb{R}^{3}\right)} \leq \exp \left(\exp \left(\exp \left(A^{C}\right)\right)\right) t^{-\frac{1}{2}} \text { for } 0<t \leq 1 .
$$

Before describing our contribution, we first find it instructive to outline Tao's approach in [47].

Fundamental to Tao's approach for showing (22) is the following fact ${ }^{16}$ (see Section 6 in [47]). There exists a universal constant $\varepsilon_{0}$ such that if $u$ is a classical solution to the Navier-Stokes equations with

$$
\begin{aligned}
& \qquad u \|_{L_{t}^{\infty} L_{x}^{3}\left(\mathbb{R}^{3} \times(0,1)\right)} \leq A \\
& \text { and } N^{-1}\left\|P_{N} u\right\|_{L_{x, t}^{\infty}\left(\mathbb{R}^{3} \times\left(\frac{1}{2}, 1\right)\right)}<\varepsilon_{0} \text { for all } N \geq N_{*},
\end{aligned}
$$

then $\|u\|_{L_{x, t}^{\infty}\left(\mathbb{R}^{3} \times\left(\frac{7}{8}, 1\right)\right)}$ can be estimated explicitly in terms of $A$ and $N_{*}$. Related observations were made previously in [11], but in a slightly different context and without the bounds explicitly stated.

With this perspective, Tao's aim is the following:

14 See footnote 6 for the definition of 'effective' bounds.

15 In [47], these are solutions that are smooth in $\mathbb{R}^{3} \times(0,1)$ and such that all derivatives of $u$ and $p$ lie in $L_{t}^{\infty} L_{x}^{2}\left(\mathbb{R}^{3} \times(0,1)\right)$.

16 Let $\varphi \in C_{0}^{\infty}\left(B_{0}(1)\right)$ with $\varphi \equiv 1$ on $B_{0}\left(\frac{1}{2}\right)$. The Littlewood-Paley projection $P_{N}$ is defined for any $N>0$ by $\widehat{P_{N}} f(\xi):=\left(\varphi\left(\frac{\xi}{N}\right)-\varphi\left(\frac{2 \xi}{N}\right)\right) \widehat{f}(\xi)$. 
Tao's goal: Under the scale-invariant assumption (23), if (24) fails for $\varepsilon_{0}=A^{-C}$ and $N=N_{0}$, what is an upper bound for $N_{0}$ ?

In [47] (Theorem $5.1 \mathrm{in}$ [47]), it is shown that $N_{0} \lesssim \exp \exp \exp \left(A^{C}\right)$, which implies (22) by means of the quantitative regularity mechanism (24) with $N_{*}=2 N_{0}$. We emphasize that since the regularity mechanism (24) is global: all quantitative estimates obtained in this way are in terms of globally defined quantities.

The strategy in [47] for showing Tao's goal with $N_{0} \lesssim \exp \left(\exp \left(\exp \left(A^{C}\right)\right)\right)$ can be summarized in four steps. We refer the reader to the Introduction in [47] for more details.

1) Frequency bubbles of concentration (Proposition 3.2 in [47]).

Suppose $\|u\|_{L_{t}^{\infty} L_{x}^{3}\left(\mathbb{R}^{3} \times\left(t_{0}-T, t_{0}\right)\right)} \leq A$ is such that

$$
N_{0}^{-1}\left|P_{N_{0}} u\left(x_{0}, t_{0}\right)\right|>A^{-C} .
$$

Then for all $n \in \mathbb{N}$ there exists $N_{n}>0,\left(x_{n}, t_{n}\right) \in \mathbb{R}^{3} \times\left(t_{0}-T, t_{n-1}\right)$ such that

$$
N_{n}^{-1}\left|P_{N_{n}} u\left(x_{n}, t_{n}\right)\right|>A^{-C}
$$

with

$$
x_{n}=x_{0}+O\left(\left(t_{0}-t_{n}\right)^{\frac{1}{2}}\right), N_{n} \sim\left|t_{0}-t_{n}\right|^{-\frac{1}{2}} .
$$

2) Localized lower bounds on vorticity (p. 37 in [47]). For certain scales $S>0$ and an 'epoch of regularity' $I_{S} \subset\left[t_{0}-S, t_{0}-A^{-\alpha} S\right]$, where the solution enjoys 'good' quantitative estimates on $\mathbb{R}^{3} \times I_{S}$ (in terms of $A$ and $S$ ), Tao shows the following: the previous step and $\|u\|_{L_{t}^{\infty} L_{x}^{3}\left(\mathbb{R}^{3} \times\left[t_{0}-T, t_{0}\right]\right)} \leq A$ imply

$$
\int_{B_{x_{0}}\left(A^{\beta} S^{\frac{1}{2}}\right)}|\omega(x, t)|^{2} d x \geq A^{-\gamma} S^{-\frac{1}{2}} \text { for all } t \in I_{S} .
$$

Here, $\alpha, \beta$ and $\gamma$ are positive universal constants.

3) Lower bound on the $L^{3}$ norm at the final moment in time $t_{0}$ (p.37-40 in [47]). Using quantitative versions of the Carleman inequalities in [13] (Propositions 4.2-4.3 in [47]), Tao shows that the lower bounds in step 2 can be transferred to a lower bound on the $L^{3}$ norm of $u$ at the final moment of time $t_{0}$. The applicability of the Carleman inequalities to the vorticity equation requires the 'epochs of regularity' in the previous step and the existence of 'good spatial annuli' where the solution enjoys good quantitative estimates. Specifically, Tao shows that step 2 on $I_{S}$ implies

$$
\int_{R_{S} \leq\left|x-x_{0}\right| \leq R_{S}^{\prime}}\left|u\left(x, t_{0}\right)\right|^{3} d x \geq \exp \left(-\exp \left(A^{C}\right)\right) .
$$

4) Conclusion: summing scales to bound $T N_{0}^{2}$. Letting $S$ vary for certain permissible $S$, the annuli in (29) become disjoint. The sum of (29) over such disjoint permissible annuli is bounded above by $\left\|u\left(\cdot, t_{0}\right)\right\|_{L^{3}\left(\mathbb{R}^{3}\right)}$ and the lower bound due to the summing of scales is $\exp \left(-\exp \left(A^{C}\right)\right) \log \left(T N_{0}^{2}\right)$. This gives the desired bound on $N_{0}$, namely

$$
T N_{0}^{2} \lesssim \exp \left(\exp \left(\exp \left(A^{C}\right)\right)\right)
$$


Let us emphasize once more that the approach in [47] produces quantitative estimates involving globally defined quantities, since the quantitative regularity mechanism (24) is inherently global. We would also like to emphasize that the fact that $\|u\|_{L_{t}^{\infty} L_{x}^{3}}<A$ is crucial for showing steps 1-2 in the above strategy.

The goal of the present paper is to develop a new robust strategy for obtaining new quantitative estimates of the Navier-Stokes equations, which are then applied to obtain Theorems A and B. The main novelty (which we explain in more detail below) is that our strategy allows us to obtain local quantitative estimates and even applies to certain situations where we are outside the regime of quantitative scale-invariant controls. For simplicity, we will outline the strategy for the case when $\|u\|_{L_{t}^{\infty} L_{x}^{3, \infty}\left(\mathbb{R}^{3} \times\left(t_{0}-T, t_{0}\right)\right)} \leq M$, before remarking on this strategy for cases without such a quantitative scale-invariant control (Theorem B).

Fundamental to our strategy is the use of local-in-space smoothing near the initial time for the Navier-Stokes equations pioneered by Jia and Šverák in [20] (see also [6] for extensions to critical cases). In particular, the result of [20], together with rescaling arguments from [6], implies the following. If $u: \mathbb{R}^{3} \times\left[t_{0}-T, t_{0}\right] \rightarrow \mathbb{R}^{3}$ is a smooth solution with sufficient decay ${ }^{17}$ of the Navier-Stokes equations and $\|u\|_{L_{t}^{\infty} L_{x}^{3, \infty}\left(\mathbb{R}^{3} \times\left(t_{0}-T, t_{0}\right)\right)} \leq$ $M$, then

$$
\int_{B_{x_{0}}\left(4{\sqrt{S^{\sharp}(M)}}^{-1}\left(t_{0}-t_{0}^{*}\right)^{\frac{1}{2}}\right)}\left|\omega\left(x, t_{0}^{*}\right)\right|^{2} d x \leq \frac{M^{2} \sqrt{S^{\sharp}(M)}}{\left(t_{0}-t_{0}^{*}\right)^{\frac{1}{2}}}
$$

for a time $t_{0}^{*} \in\left(t_{0}-T, t_{0}\right)$ implies that

$$
\|u\|_{L_{x, t}^{\infty}}\left(B_{x_{0}}\left(\frac{1}{2}{\sqrt{S^{\sharp}(M)}}^{-1}\left(t_{0}-t_{0}^{*}\right)^{\frac{1}{2}}\right) \times\left(\frac{3}{4}\left(t_{0}-t_{0}^{*}\right)+t_{0}^{*}, t_{0}\right)\right.
$$

can be estimated explicitly in terms of $M$ and $t_{0}-t_{0}^{*}$. Here, $S^{\sharp}(M)=C M^{-100}$ is defined in (76).

With this perspective, the aim of our strategy is the following

Our goal: If (30) fails for $t_{0}^{*}=t_{0}^{\prime}$, what is a lower bound for $t_{0}-t_{0}^{\prime}$ ?

This is the main aim of Proposition 2.1. Taking $s_{0}$ such that $t_{0}-t_{0}^{\prime} \geq 2 T s_{0}$, we can then apply (30)-(31) with $t_{0}^{*}=t_{0}-T s_{0}$. One might think of the main goal of our strategy as a physical space analogy to Tao's goal with

$$
N_{0} \sim\left|t_{0}-t_{0}^{\prime}\right|^{-\frac{1}{2}}
$$

In contrast to (24), the regularity mechanism (30)-(31) produces quantitative estimates that are in terms of locally defined quantities, which is crucial for obtaining the localized results as in Theorem A. Our strategy for obtaining a lower bound of $t_{0}-t_{0}^{\prime}$ (see Proposition 2.1) can be summarized in three steps; see also Fig. 1.

1) Backward propagation of vorticity concentration (Lemma 3.1).

Let $\|u\|_{L_{t}^{\infty} L_{x}^{3, \infty}\left(\mathbb{R}^{3} \times\left(t_{0}-T, t_{0}\right)\right)} \leq M$. Suppose $t_{0}^{\prime} \in\left(t_{0}-T, t_{0}\right)$ is not too close to $t_{0}-T$ and is such that

$$
\int_{B_{x_{0}}\left(4{\sqrt{S^{\sharp}(M)}}^{-1}\left(t_{0}-t_{0}^{\prime}\right)^{\frac{1}{2}}\right)}\left|\omega\left(x, t_{0}^{\prime}\right)\right|^{2} d x>\frac{M^{2} \sqrt{S^{\sharp}(M)}}{\left(t_{0}-t_{0}^{\prime}\right)^{\frac{1}{2}}} .
$$

17 In this paper, 'smooth solution with sufficient decay' always denotes the notion described in Sect. 1.4. 


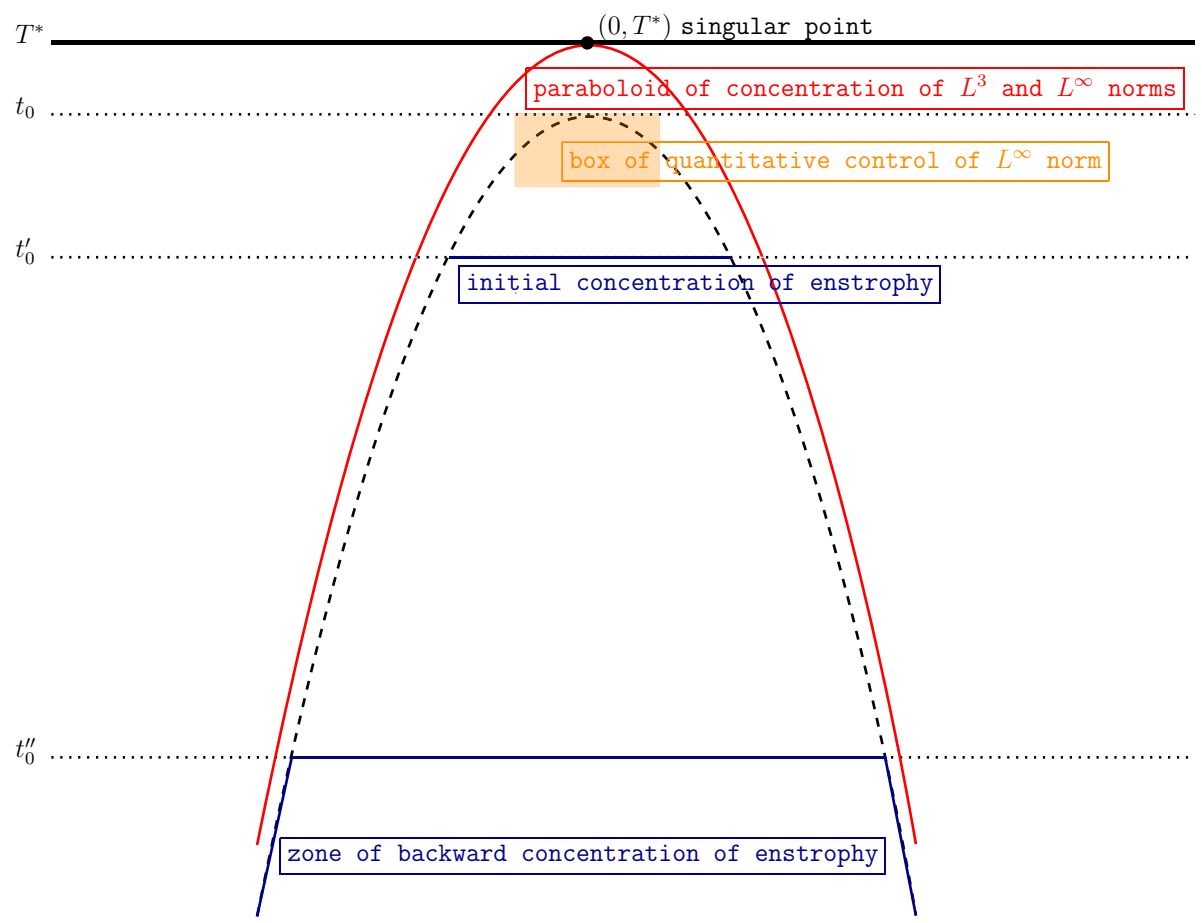

Fig. 1. Zones of concentration and quantitative regularity

We show that for all $t_{0}^{\prime \prime} \in\left(t_{0}-T, t_{0}^{\prime}\right)$, such that $t_{0}-t_{0}^{\prime \prime}$ is sufficiently large compared to $t_{0}-t_{0}^{\prime}$ (in other words $t_{0}^{\prime \prime}$ is well-separated from $t_{0}^{\prime}$ ), we have

$$
\int_{B_{x_{0}}\left(4{\sqrt{S^{\sharp}(M)}}^{-1}\left(t_{0}-t_{0}^{\prime \prime}\right)^{\frac{1}{2}}\right)}\left|\omega\left(x, t_{0}^{\prime \prime}\right)\right|^{2} d x>\frac{M^{2} \sqrt{S^{\sharp}(M)}}{\left(t_{0}-t_{0}^{\prime \prime}\right)^{\frac{1}{2}}} .
$$

We refer the reader to Lemma 3.1 for precise statements for the rescaled/translated situation $\mathbb{R}^{3} \times\left(t_{0}-T, t_{0}\right)=\mathbb{R}^{3} \times(-1,0)$.

2) Lower bound on localized $L^{3}$ norm at the final moment in time $t_{0}$. Using the previous step, together with the same arguments as [47] involving quantitative Carleman inequalities, we show that for certain permissible annuli that

$$
\int_{R \leq\left|x-x_{0}\right| \leq R^{\prime}}\left|u\left(x, t_{0}\right)\right|^{3} d x \geq \exp \left(-\exp \left(M^{C}\right)\right) .
$$

We wish to mention that the role of the Type I bound is to show the solution $u$ obeys good quantitative estimates in certain space-time regions, which is needed to apply the Carleman inequalities to the vorticity equation.

3) Conclusion: summing scales to bound $t_{0}-t_{0}^{\prime}$ from below. Summing (34) over all permissible disjoint annuli finally gives us the desired lower bound for $t_{0}-t_{0}^{\prime}$ in Proposition 2.1. We note that the localized $L^{3}$ norm of $u$ at time $t_{0}$ plays a distinct role 
to that of Type I condition described in the previous step. Its sole purpose is to bound the number of permissible disjoint annuli that can be summed, which in turn gives the lower bound of $t_{0}-t_{0}^{\prime}$. Together with the assumed global Type I assumption, this is essentially why the lower bound in Theorem A on the localized $L^{3}$ norm near a Type I singularity is a single logarithm and holds at pointwise times.

Although the above relates to the case of Proposition 2.1 and Theorem A where

$$
\|u\|_{L_{t}^{\infty} L_{x}^{3, \infty}\left(\mathbb{R}^{3} \times\left(t_{0}-T, t_{0}\right)\right)} \leq M,
$$

we stress that the above strategy (with certain adjustments) is robust enough to apply to certain settings without a quantitative Type I control (Theorem B).

Recall that Theorem $\mathrm{B}$ is concerned with quantitative estimates on $u: \mathbb{R}^{3} \times(-1,0) \rightarrow$ $\mathbb{R}^{3}$, where we assume

$$
t_{(k)} \uparrow 0 \text { with } \sup _{k}\left\|u\left(\cdot, t_{(k)}\right)\right\|_{L^{3}\left(\mathbb{R}^{3}\right)} \leq M .
$$

First we remark that the local quantitative regularity statement (30)-(31) remains true (with $t_{0}^{*}$ replaced by $t_{k}$ ) if $u$ is a $C^{\infty}\left(\mathbb{R}^{3} \times(-1,0]\right)$ finite-energy solution and the Type I condition is replaced by the weaker assumption that $\left\|u\left(\cdot, t_{(k)}\right)\right\|_{L^{3}\left(\mathbb{R}^{3}\right)} \leq M$. Our goal then becomes the following

Our second goal: If (30) fails for $t_{0}^{*}=t_{j}$ (with $t_{0}=0$ and $T=1$ ), what is an upper bound for $j$ ?

In this setting '1) Backward propagation of vorticity concentration' still remains valid if a sufficiently well-separated subsequence of $t_{(k)}$ is taken (see Lemma 3.3 and Proposition 2.2). To show this we use energy estimates in [40] for solutions to the NavierStokes equations with $L^{3}\left(\mathbb{R}^{3}\right)$ initial data. Such estimates are also central to gain good quantitative control of the solution in certain space-time regions, which are required for applying the quantitative Carleman inequalities. The price one pays in this setting (when compared to the estimates in [47]), is a gain of an additional exponential in the estimates. The reason is the control on the energy of $u(\cdot, t)-e^{t \Delta} u_{0}$ ( with $u_{0} \in L^{3}\left(\mathbb{R}^{3}\right)$ ) requires the use of Gronwall's lemma.

In the strategy in [47] the lower bound on vorticity (28), which is needed for getting a lower bound on the localized $L^{3}$ norm at $t_{0}$ via quantitative Carleman inequalities, is obtained from the frequency bubbles of concentration. In order for this transfer of scaleinvariant information to take place, it appears essential that the solution has a quantitative scale-invariant control such as $\|u\|_{L_{t}^{\infty} L^{3}} \leq A$. In our strategy, we instead work directly with quantities involving vorticity (similar to (28)), which are tailored for the immediate use of quantitative Carleman inequalities. In this way, we crucially avoid any need to transfer scale-invariant information, giving our strategy a certain degree of robustness.

1.2. Final Remarks and Comments. We give some heuristics about the quantitative estimates of the form

$$
\|u\|_{L^{\infty}\left(\mathbb{R}^{3} \times\left(\frac{1}{2}, 1\right)\right)} \leq G\left(\|u\|_{X}\right)
$$

that one can expect for the Navier-Stokes equations, when a finite-energy solution $u$ solution belongs to certain normed spaces $X \subset L^{1}((0,1) ; Y)$, where $Y$ is a Banach space contained in $\mathcal{S}^{\prime}\left(\mathbb{R}^{3}\right)$. 
1.2.1. Subcritical case Consider a space $X \subset \mathcal{S}^{\prime}\left(\mathbb{R}^{3} \times(0,1)\right)$ whose norm $\|\cdot\|_{X}$ is subcritical ${ }^{18}$ (for example $L_{x, t}^{5+\delta}\left(\mathbb{R}^{3} \times(0,1)\right)$ with $\delta>0$ ). If $u$ is a finite-energy solution with a finite subcritical norm on $\mathbb{R}^{3} \times(0,1)$, then it is known that $u$ must belong to $C^{\infty}\left(\mathbb{R}^{3} \times(0,1]\right)$. See, for example, [24]. Moreover, one typically has a quantitative estimate of the form (36) with

$$
G(x)=c x^{\beta} \text { with } \beta>0 .
$$

To demonstrate this, consider $u$ belong to $L_{x, t}^{5+\delta}\left(\mathbb{R}^{3} \times(0,1)\right)$. An application of Caffarelli, Kohn and Nirenberg's result [9] (see also Proposition 6.1) gives that (36) holds true with $G(x) \sim x^{\frac{\delta+5}{\delta}}$. Such a quantitative estimate is invariant with respect to the Navier-Stokes scaling (2). In this context, one could also gain similar quantitative estimates based on parabolic bootstrap arguments applied to the vorticity equation

$$
\partial_{t} \omega-\Delta \omega=\omega \cdot \nabla u-u \cdot \nabla \omega, \quad \omega=\nabla \times u
$$

as was done by Serrin in [42].

1.2.2. Critical case In the subcritical norm case, we saw that seeking estimates of the form (36) that are invariant with respect to the scaling (2), gives a suitable candidate for $G$ that can be realised. The case when the norm $\|\cdot\|_{X}$ is critical is more subtle, since a scaling argument does not provide a suitable candidate for $G$. We first mention that the case of sufficiently small critical norms, for example

$$
\|u\|_{L^{5}\left(\mathbb{R}^{3} \times(0,1)\right)}<\varepsilon_{0},
$$

is essentially of a similar category to the subcritical case (though a scaling argument is not applicable). Indeed, a similar argument outlined as before (based on [9], see also Proposition 6.1) gives that in this case we have (36) with $G(x) \sim x$. This is consistent with the fact that solutions with small scale-invariant norms exhibit similar behaviour to the linear system and hence are typically expected to satisfy linear estimates.

For obtaining quantitative estimates of the form (36) when the scale-invariant norm is large, it is less clear what the candidate for $G$ might be. This seems to be the case even for large global scale-invariant norms that exhibit smallness at small local scales ${ }^{19}$ (for example $\left.L^{5}\left(\mathbb{R}^{3} \times(0,1)\right)\right)$. Such local smallness properties have been utilized to prove qualitative regularity by essentially linear methods. See [45], for example.

For the case of a smooth finite-energy solution $u$ having finite scale-invariant $L^{5}\left(\mathbb{R}^{3} \times\right.$ $(0,1))$ norm, one way to obtain quantitative estimates ${ }^{20}$ is to consider the vorticity equation (37) with initial vorticity $\omega_{0} \in L^{2}\left(\mathbb{R}^{3}\right)$. Performing an energy estimate yields for $t \in[0,1]$

$$
\|\omega(\cdot, t)\|_{L^{2}\left(\mathbb{R}^{3}\right)}^{2}+2 \int_{0}^{t} \int_{\mathbb{R}^{3}}|\nabla \omega|^{2} d x d t^{\prime}=\left\|\omega_{0}\right\|_{L^{2}\left(\mathbb{R}^{3}\right)}^{2}+2 \int_{0}^{t} \int_{\mathbb{R}^{3}}(\omega \cdot \nabla u) \cdot \omega d x d t^{\prime},
$$

18 See Sect. 1.4 for a definition.

19 In particular, $u \in L^{5}\left(\mathbb{R}^{3} \times(0,1)\right) \Rightarrow \lim _{r \downarrow 0}\|u\|_{L^{5}\left(B_{0}(r) \times\left(1-r^{2}, 1\right)\right)}=0$.

20 For very similar computations, see (for example) Chapter 11 of [26] and references therein. 
where the second term in right-hand side is due to the vortex stretching term $\omega \cdot \nabla u$ in (37). For the case that $u \in L^{5}\left(\mathbb{R}^{3} \times(0,1)\right)$, application of Hölder's inequality, Sobolev embedding theorems and Young's inequality lead to

$$
\|\omega(\cdot, t)\|_{L^{2}\left(\mathbb{R}^{3}\right)}^{2}+\int_{0}^{t} \int_{\mathbb{R}^{3}}|\nabla \omega|^{2} d x d t^{\prime} \leq\left\|\omega_{0}\right\|_{L^{2}\left(\mathbb{R}^{3}\right)}^{2}+C \int_{0}^{t}\left\|u\left(\cdot, t^{\prime}\right)\right\|_{L^{5}\left(\mathbb{R}^{3}\right)}^{5}\left\|\omega\left(\cdot, t^{\prime}\right)\right\|_{L^{2}\left(\mathbb{R}^{3}\right)}^{2} d t^{\prime} .
$$

Gronwall's lemma, followed by arguments similar to the subcritical case, yields

$$
\|u\|_{L^{\infty}\left(\mathbb{R}^{3} \times\left(\frac{1}{2}, 1\right)\right)} \lesssim\|\omega\|_{L^{\infty}\left(0,1 ; L^{2}\left(\mathbb{R}^{3}\right)\right)}^{2} \leq\left\|\omega_{0}\right\|_{L^{2}\left(\mathbb{R}^{3}\right)}^{2} \exp \left(\|u\|_{L^{5}\left(\mathbb{R}^{3} \times(0,1)\right)}^{5}\right) .
$$

Though this is not exactly of the form (36), a slightly different argument gives that for any finite-energy solution $u$ in $L^{5}\left(\mathbb{R}^{3} \times(0,1)\right)$ we get that (36) holds with $G(x) \sim \exp \left(C x^{5}\right)$. In particular, this can be achieved using $L^{q}$ energy estimates in [31], the pigeonhole principle and reasoning in the previous subsection.

The above argument (39)-(41) shows that being able to substantially improve upon $G(x) \sim \exp \left(C x^{5}\right)$ would most likely require the utilization of a nonlinear mechanism that reduces the influence of the vortex stretching term $\omega \cdot \nabla u$ in (37). It seems plausible that the discovery of such a mechanism would have implications for the regularity theory of the Navier-Stokes equations.

1.3. Outline of the paper. In each of the Sects. 2-7, we distinguish between cases where: (i) one assumes a Type I control on the solution and (ii) one assumes a control on the velocity field on time slices only.

In Sect. 2, we state our main quantitative estimates (Propositions 2.1 and 2.2) and we demonstrate how these statements imply the main results of this paper: Theorem A, Corollary 1.1 and Theorem B. Section 3 is devoted to the proof of Propositions 2.1 and 2.2. Section 4 contains three further applications of the technology developed in the present paper, in particular Corollary 4.3 concerning a quantitative bound for the number of singularities in a Type I blow-up scenario. In Sect. 5, we quantify Jia and Šverák's results regarding local-in-space short-time smoothing, which is a main tool for proving the quantitative estimates in Sect. 3. The main result in Sect. 5 is Theorem 5.1. In Sect. 6, we give a new proof of Tao's result that solutions possess 'quantitative annuli of regularity', which is required for proving our main propositions in Sect. 3. The central results in Sect. 6 are Lemmas 6.6 and 6.8. Section 7 is concerned with the utilization of arguments from the papers of Leray and Tao to show existence of quantitative epochs of regularity (Lemmas 7.3 and 7.5). In "Appendix A" we recall known results about mild solutions and local energy solutions, and we give pressure formulas. In "Appendix B", we recall the quantitative Carleman inequalities proven by Tao.

At this point we find it useful to give the high-level structure of the proofs of the main results, Theorems A and B, stated in the Introduction. These results are proved in Sect. 2.2 so as to emphasize the link stated in Sect. 2.1 between concentration and quantitative regularity estimates.

The proof of Theorem A relies on the combination (as is showed in Fig. 1) of the quantitative bound in the Type I case (Proposition 2.1) on the one hand, with concentration estimates near a potential singularity for the local $L^{3}$ norm ( [6]) and for the $L^{\infty}$ norm (Corollary 5.3) on the other hand. The proof of Theorem B directly follows from the quantitative bound in the time slice case (Proposition 2.2) and local-in-space smoothing results (Theorem 5.1). 
Hence, the core of the paper are the proofs of the main quantitative estimates, Proposition 2.1 and Proposition 2.2. Their proofs in Sect. 3 rely on:

- backward propagation of concentration stated in Lemma 3.1 (Type I) and Lemma 3.3 (time slice) via quantitative local-in-space short-time smoothing stated in Theorem 5.1 and Remark 5.2,

- large-scale propagation of concentration via quantitative unique continuation in epochs of regularity,

- forward propagation of concentration via quantitative backward uniqueness in annuli of regularity

- and summing of scales.

The auxiliary tools of quantitative annuli of regularity are proved in Sect. 6 and of quantitative epochs of regularity are proved in Sect. 7. The Carleman inequalities for quantitative unique continuation and quantitative backward uniqueness are taken directly from Tao's paper [47] and stated in "Appendix B".

1.4. Notations. In order to make it easier to locate the statements of the results, we have adopted the following convention. The two main theorems of the paper are Theorems A and Theorem B. They are located in the Introduction. The other theorems, propositions, corollaries and remarks are numbered as ' $a . b$ ', where $a$ is the section number and $b$ the ordinal number of the statement in that section.

1.4.1. Universal constants For universal constants in the statements of propositions and lemmas associated to the Type I case (specifically Proposition 2.1 and Lemma 3.1), we adopt the convention of a superscript $\sharp$. For universal constants in the statements of propositions and lemmas associated to the time slices case (specifically Proposition 2.2 and Lemma 3.3), we adopt the convention of a superscript $b$.

In Lemmas 3.1, 3.3 and Sect. 5, we track the numerical constants arising. Elsewhere in this paper, we adopt the convention that $C$ denotes a positive universal constant which may vary from line to line.

We use the notation $X \lesssim Y$, which means that there exists a positive universal constant $C$ such that $X \leq C Y$.

In several places in this paper (notably the Introduction, Sect. 3 and "Appendix B") the notation $C$ is used to denote a positive universal constant and $-C$ denotes a negative universal constant.

Whenever we refer to a quantity ( $M$ for example) being 'sufficiently large', we understand this as $M$ being larger than some universal constant that can (in principle) be specified.

\subsubsection{Vectors and domains For a vector $a, a_{i}$ denotes the $i^{\text {th }}$ component of $a$.}

For $(x, t) \in \mathbb{R}^{4}$ and $r>0$ we denote $B_{x}(r):=\left\{y \in \mathbb{R}^{3}:|y-x|<r\right\}$ and $Q_{(x, t)}(r):=B_{r}(x) \times\left(t-r^{2}, t\right)$. Here, $|\cdot|$ denotes the Euclidean metric. As is usual, for $a, b \in \mathbb{R}^{3},(a \otimes b)_{\alpha \beta}=a_{\alpha} b_{\beta}$, and for $A, B \in M_{3}(\mathbb{R}), A: B=A_{\alpha \beta} B_{\alpha \beta}$. Here and in the whole paper we use Einstein's convention on repeated indices. For $F: \Omega \subseteq \mathbb{R}^{3} \rightarrow \mathbb{R}^{3}$, we define $\nabla F \in M_{3}(\mathbb{R})$ by $(\nabla F(x))_{\alpha \beta}:=\partial_{\beta} F_{\alpha}$.

Let us stress that in Sect. 5 only we use cubes instead of balls: $B_{x}(r)=x+(-r, r)^{3}$. This is for computational convenience, since we track numerical constants in Sect. 5. We emphasize that the results in Sect. 5 hold for spherical balls too, with certain universal constants adjusted. 
1.4.3. Blow-up point, criticality We say that a solution $u$ to the Navier-Stokes equations first blows-up at $T^{*}>0$ if

$$
u \in L_{l o c}^{\infty}\left(\left(0, T^{*}\right), L^{\infty}\left(\mathbb{R}^{3}\right)\right)
$$

but

$$
u \notin L_{l o c}^{\infty}\left(\left(0, T^{*}\right] ; L^{\infty}\left(\mathbb{R}^{3}\right)\right) .
$$

We say $\left(X,\|\cdot\|_{X}\right) \subset \mathcal{S}^{\prime}\left(\mathbb{R}^{3}\right)$ is critical if $u_{0} \in X \Rightarrow u_{0 \lambda}(x):=\lambda u_{0}(\lambda x) \in X$ with $X$ norm equal to that of $u_{0}$.

A quantity $F(u, p) \in[0, \infty)$ is said to be subcritical if, for the rescaling (2), there exists $\alpha>0$ such that $F\left(u_{\lambda}, p_{\lambda}\right)=\lambda^{\alpha} F(u, p)$, critical if we have $F\left(u_{\lambda}, p_{\lambda}\right)=F(u, p)$ and supercritical if we have $F\left(u_{\lambda}, p_{\lambda}\right)=\lambda^{-\beta} F(u, p)$ for some $\beta>0$.

1.4.4. Mild, suitable and finite-energy solutions to the Navier-Stokes equations Throughout this paper, we refer to $u: \mathbb{R}^{3} \times[0, T] \rightarrow \mathbb{R}^{3}$ as a mild solution of the Navier-Stokes equations (1) if $u \in \cap_{t<T} L^{2}\left(\left(0, t_{1}\right) ; L_{u l o c}^{2}\left(\mathbb{R}^{3}\right)\right)$ and if it satisfies the Duhamel formula:

$$
u(x, t)=e^{t \Delta} u(\cdot, 0)+\int_{0}^{t} \mathbb{P} \partial_{i} e^{(t-s) \Delta} u_{i}(\cdot, s) u_{j}(\cdot, s) d s,
$$

for all $t \in[0, T]$. Here, $e^{t \Delta}$ is the heat semigroup, $\mathbb{P}$ is the projection onto divergencefree vector fields. A mild solution on $\left[0, T^{*}\right)$ is a function that is a mild solution on $[0, T]$ for any $T \in\left(0, T^{*}\right)$.

We say that $u$ is a smooth solution with sufficient decay on the interval $\left[0, T^{*}\right]$ if $u$ is smooth on the epoch $(0, T)$ for any $T<T^{*}$ and belongs to $L^{\infty}\left((0, T) ; L^{4}\left(\mathbb{R}^{3}\right)\right) \cap$ $L^{\infty}\left((0, T) ; L^{5}\left(\mathbb{R}^{3}\right)\right)$. Furthermore using Lemma 2.4 in [19], it gives that $u$ coincides with all local energy solutions (we refer to the final paragraph of Sect. 1.4.4 for a definition), with initial data $u(\cdot, s)$, on $\mathbb{R}^{3} \times\left(s, T^{*}\right)$ for any $0<s<T^{*}$. The framework of 'smooth solutions with sufficient decay' is needed to apply Theorem A to the setting of Corollary 1.1 , where the solution is not of finite energy. A mild solution with Schwartz class initial data and maximal time of existence $T^{*}$ will be such a solution, and so Theorem A applies to that setting. Notice that smoothness is needed here in order to get estimate (10) for all $t$ in the ad hoc interval.

We say $u$ is a finite-energy solution or a Leray-Hopf solution to the Navier-Stokes equations on $(0, T)$ if $u \in C_{w}\left([0, T] ; L_{\sigma}^{2}\left(\mathbb{R}^{3}\right)\right) \cap L^{2}\left(0, T ; \dot{H}^{1}\left(\mathbb{R}^{3}\right)\right)$ and if it satisfies the global energy inequality

$$
\|u(\cdot, t)\|_{L^{2}\left(\mathbb{R}^{3}\right)}^{2}+2 \int_{0}^{t} \int_{\mathbb{R}^{3}}|\nabla u|^{2} d x d t^{\prime} \leq\|u(\cdot, 0)\|_{L^{2}\left(\mathbb{R}^{3}\right)}^{2} .
$$

Let $\Omega \subseteq \mathbb{R}^{3}$. We say that $(u, p)$ is a suitable weak solution to the Navier-Stokes equations (1) in $\Omega \times\left(T_{1}, T\right)$ if it fulfills the properties described in [38] (Definition 6.1 p.133 in [38]).

We say that $(u, p)$ is a suitable finite-energy solution to the Navier-Stokes equations on $\mathbb{R}^{3} \times\left(T_{1}, T\right)$ if it is a solution to (1) in the sense of distributions and

- $u \in C_{w}\left(\left[T_{1}, T\right] ; L_{\sigma}^{2}\left(\mathbb{R}^{3}\right)\right) \cap L_{t}^{2}\left(T_{1}, T ; \dot{H}^{1}\left(\mathbb{R}^{3}\right)\right)$, 
- it satisfies the global energy inequality

$$
\|u(\cdot, t)\|_{L^{2}}^{2}+2 \int_{T_{1}}^{t} \int_{\mathbb{R}^{3}}|\nabla u|^{2} d y d s \leq\left\|u\left(\cdot, T_{1}\right)\right\|_{L^{2}}^{2} \text { for all } t \in\left[T_{1}, T\right],
$$

- $(u, p)$ is a suitable weak solution on $B_{1}(x) \times\left(T_{1}, T\right)$ for all $x \in \mathbb{R}^{3}$.

It is known, see for instance [2], that the above defining properties of a suitable finiteenergy solution imply that there exists $\Sigma \subset\left[T_{1}, T\right]$ with full Lebesgue measure $|\Sigma|=$ $T-T_{1}$ such that

$$
\begin{array}{r}
\|u(\cdot, t)\|_{L^{2}}^{2}+2 \int_{t^{\prime}}^{t} \int_{\mathbb{R}^{3}}|\nabla u|^{2} d y d s \leq\left\|u\left(\cdot, t^{\prime}\right)\right\|_{L^{2}}^{2} \text { and }\left\|\nabla u\left(\cdot, t^{\prime}\right)\right\|_{L^{2}}<\infty, \\
\text { for all } t \in\left[t^{\prime}, T\right] \text { and } t^{\prime} \in \Sigma .
\end{array}
$$

Finally, let us give the definition of a local energy solution to the Navier-Stokes equations. Notice that these solutions are sometimes described in the literature as 'LemariéRieusset solutions' or 'Leray solutions'. They were conceived by Lemarié-Rieusset in [25]. In our paper, whenever we refer to 'local energy solutions', we mean in the sense of Definition 2.1 in [19]. Notice, in particular, that suitable finite-energy solutions are local energy solutions.

1.4.5. Lorentz spaces For a measurable subset $\Omega \subseteq \mathbb{R}^{d}$ and a measurable function $f: \Omega \rightarrow \mathbb{R}$ we define

$$
d_{f, \Omega}(\alpha):=\mu(\{x \in \Omega:|f(x)|>\alpha\}),
$$

where $\mu$ denotes the Lebesgue measure. The Lorentz space $L^{p, q}(\Omega)$, with $p \in[1, \infty)$, $q \in[1, \infty]$, is the set of all measurable functions $g$ on $\Omega$ such that the quasinorm $\|g\|_{L^{p, q}(\Omega)}$ is finite. The quasinorm is defined by

$$
\begin{aligned}
& \|g\|_{L^{p, q}(\Omega)}:=\left(p \int_{0}^{\infty} \alpha^{q} d_{g, \Omega}(\alpha)^{\frac{q}{p}} \frac{d \alpha}{\alpha}\right)^{\frac{1}{q}}, \\
& \|g\|_{L^{p, \infty}(\Omega)}:=\sup _{\alpha>0} \alpha d_{g, \Omega}(\alpha)^{\frac{1}{p}} .
\end{aligned}
$$

Notice that for $p \in(1, \infty)$ and $q \in[1, \infty]$ there exists a norm, which is equivalent to the quasinorm defined above, for which $L^{p, q}(\Omega)$ is a Banach space. For $p \in[1, \infty)$ and $1 \leq q_{1}<q_{2} \leq \infty$, we have the following continuous embeddings

$$
L^{p, q_{1}}(\Omega) \hookrightarrow L^{p, q_{2}}(\Omega)
$$

and the inclusion is strict. 


\section{Main Quantitative Estimates}

2.1. Quantitative estimates in the Type I and time slices case.

Proposition 2.1 (main quantitative estimate, Type I). There exists a universal constant $M_{2} \in[1, \infty)$ such that the following holds. Let $M \in\left[M_{2}, \infty\right), t_{0} \in \mathbb{R}$ and $T \in(0, \infty)$. There exists $S^{\sharp}(M) \in\left(0, \frac{1}{4}\right]$, such that the following holds. Let $(u, p)$ be a smooth solution with sufficient decay ${ }^{21}$ to the Navier-Stokes equations (1) in $I=\left[t_{0}-T, t_{0}\right]$, which satisfies

$$
\|u\|_{L_{t}^{\infty} L_{x}^{3, \infty}\left(\mathbb{R}^{3} \times\left(t_{0}-T, t_{0}\right)\right)} \leq M .
$$

and for fixed $\lambda \in\left(0, \exp \left(M^{1023}\right)\right)$

$$
\int_{B_{0}\left(\lambda T^{\frac{1}{2}}\right)}\left|u\left(x, t_{0}\right)\right|^{3} d x \geq \frac{3}{2} \exp \left(-\exp \left(M^{1024}\right)\right) .
$$

Then for

$$
-s_{\lambda}:=\frac{C^{\sharp} \lambda^{2}}{16} M^{-749} \exp \left\{-4 M^{1023} \exp \left(\exp \left(M^{1024}\right)\right) \int_{B_{0}\left(\lambda T^{\frac{1}{2}}\right)}\left|u\left(x, t_{0}\right)\right|^{3} d x\right\},
$$

we have the bound

$$
\int_{B_{0}\left(4{\sqrt{S^{\sharp}}}^{-1}\left(-S_{\lambda} T\right)^{\frac{1}{2}}\right)}\left|\omega\left(x, t_{0}+s_{\lambda} T\right)\right|^{2} d x \leq M^{2}\left(-s_{\lambda} T\right)^{-\frac{1}{2}} \sqrt{S^{\sharp}}
$$

and the bound

$$
\|u\|_{L^{\infty}\left(B_{0}\left(C_{2} T^{\frac{1}{2}} M^{50}\left(-s_{\lambda}\right)^{\frac{1}{2}}\right) \times\left(\frac{s_{\lambda} T}{4}+t_{0}, t_{0}\right)\right)} \leq \frac{C_{1} M^{-23}}{\left(-s_{\lambda}\right)^{\frac{1}{2}} T^{\frac{1}{2}}},
$$

for universal constants $C_{1}, C_{2} \in(0, \infty)$. Here $C^{\sharp}$ and $S^{\sharp}(M)$ are the constants given by Lemma 3.1. Recall that we have $S^{\sharp}(M)=C M^{-100}$.

Figure 1 illustrates Proposition 2.1.

Proposition 2.2 (main quantitative estimate, time slices). There exists a universal constant $M_{1} \in[1, \infty)$ such that the following holds. Let $M \in\left[M_{1}, \infty\right)$. We define $M^{b}$ by (17). Let $(u, p)$ be a $C^{\infty}\left(\mathbb{R}^{3} \times(-1,0)\right)$ finite-energy solution to the Navier-Stokes equations (1) in $I=[-1,0]$. Assume that there exists $t_{(k)} \in[-1,0)$ such that

$$
t_{(k)} \uparrow 0 \text { with } \sup _{k}\left\|u\left(\cdot, t_{(k)}\right)\right\|_{L^{3}\left(\mathbb{R}^{3}\right)} \leq M .
$$

Select any “well-separated" subsequence (still denoted $\left.t_{(k)}\right)$ such that

$$
\sup _{k} \frac{-t_{(k+1)}}{-t_{(k)}}<\exp \left(-2\left(M^{b}\right)^{1223}\right) \text {. }
$$

\footnotetext{
21 See Sect. 1.4. Notice that by this definition $u$ is bounded up to $t_{0}$.
} 
For this well-separated subsequence, assume that there exists $j+1$ such that the vorticity concentrates at time $t_{(j+1)}$ in the following sense

$$
\int_{B_{0}\left(4{\sqrt{S^{b}}}^{-1}\left(-t_{(j+1)}\right)^{\frac{1}{2}}\right)}\left|\omega\left(x, t_{(j+1)}\right)\right|^{2} d x>M^{2}\left(-t_{(j+1)}\right)^{-\frac{1}{2}} \sqrt{S^{b}},
$$

where $S^{b}(M) \in\left(0, \frac{1}{4}\right]$ is as in Lemma 3.3. Then, we have the following upper bound on $j:$

$$
j \leq \exp \left(\exp \left(\left(M^{b}\right)^{1224}\right)\right)
$$

Here $S^{b}(M)$ is the constant given by Lemma 3.3.

2.2. Proofs of the main results. In this section we prove the main results stated in the Introduction. We refer to the end of Sect. 1.3 for a high-level explanation of the structure of the proofs.

Proof of Theorem A. Take $M \geq \max \left(M_{2}, M_{6}\right) \geq 1$. Here, $M_{2}$ being from Proposition 2.1 and $M_{6}$ being from Corollary 5.3. By means of a scaling argument, it is sufficient to prove Theorem A with $T^{*}=1$. In particular, we fix

$$
\begin{aligned}
& t \in\left(\frac{1}{2}, 1\right) \text { and } \\
& R \in\left(2 \sqrt{\frac{1-t}{S_{B P}(M)}}, e^{M^{1022}}\right)
\end{aligned}
$$

and assume the contrapositive of (10) with $T^{*}=1$. Namely, we assume

$$
\int_{|x|<R}|u(x, t)|^{3} d x<\frac{\log \left(\frac{R^{2}}{M^{802}|1-t|}\right)}{\exp \left(\exp \left(M^{1025}\right)\right)} .
$$

First we note that [6] (specifically Theorem 1 in [6]; see also [21, Corollary 1.2]), together with assumptions (1)-(2) in the statement of Theorem A (with $T^{*}=1$ ) imply that there exist $S_{B P}(M)$ and $\gamma_{u n i v}>0$ such that

$$
\int_{B_{0}\left(2 \sqrt{\frac{1-s}{s_{B P}(M)}}\right)}|u(x, s)|^{3} d x \geq \gamma_{\text {univ }}^{3} \text { for all } s \in(0,1) .
$$

Next define $\lambda:=\frac{R}{\sqrt{t}}$. Then by (57)-(58), we see that

$$
\lambda \in\left(0, \sqrt{2} e^{M^{1022}}\right) .
$$

Furthermore, (60) implies that for $M$ sufficiently large, we have

$$
\int_{B_{0}\left(t^{\frac{1}{2}} \lambda\right)}|u(x, t)|^{3} d x \geq \frac{3}{2} \exp \left(-\exp \left(M^{1024}\right)\right) .
$$


Considering $u$ on $\mathbb{R}^{3} \times[0, t]$ and observing Proposition 2.1, we see that (62) implies that the assumption (49) is satisfied with $T:=t$ and $t_{0}:=t$. Furthermore, we have that for $M$ sufficiently large $\lambda \in\left(0, \exp \left(M^{1023}\right)\right)$. Hence, we can apply Proposition 2.1. Namely by (52) we see that for

$$
-s_{\lambda}:=\frac{C^{\sharp} \lambda^{2}}{16} M^{-749} \exp \left\{-4 M^{1023} \exp \left(\exp \left(M^{1024}\right)\right) \int_{B_{0}(R)}|u(x, t)|^{3} d x\right\},
$$

we have the bound

$$
\|u(\cdot, t)\|_{L^{\infty}\left(B_{0}\left(C_{2} t^{\frac{1}{2}} M^{50}\left(-s_{\lambda}\right)^{\frac{1}{2}}\right)\right)} \leq \frac{C_{1} M^{-23}}{\left(-s_{\lambda}\right)^{\frac{1}{2}} t^{\frac{1}{2}}} .
$$

Using that $(0,1)$ is a singular point of $u$, the Type I bound on $u$ and Corollary 5.3, we see that there exists a universal constant $C_{\text {univ }}$ such that

$$
\|u(\cdot, t)\|_{L^{\infty}\left(B_{0}\left(\frac{2 M^{50}}{C_{\text {univ }}}(1-t)^{\frac{1}{2}}\right)\right)}>\frac{C_{\text {univ }} M^{-49}}{(1-t)^{\frac{1}{2}}} .
$$

The assumption (59) implies that for $M$ sufficiently large, we have the following lower bound for $-s_{\lambda}$ :

$$
-s_{\lambda} \geq \frac{C^{\sharp}}{16} M^{53}|1-t| .
$$

Now, (63) and (65) (together with the fact that $t>\frac{1}{2}$ ) imply that

$$
\|u(\cdot, t)\|_{L^{\infty}\left(B_{0}\left(C_{2} 2^{-\frac{1}{2}} M^{50}\left(\frac{C^{\sharp}}{16} M^{53}|1-t|\right)^{\frac{1}{2}}\right)\right)} \leq 2^{\frac{1}{2}} C_{1} M^{-23}\left(\frac{16}{C^{\sharp} M^{53}|1-t|}\right)^{\frac{1}{2}} .
$$

For $M$ sufficiently large, this contradicts (64). Hence, (59) cannot hold.

Proof of Corollary 1.1. From [10] (specifically Theorem 1.1 in [10]), there exists $M>1$ such that

$$
|u(x, t)| \leq \frac{M}{|x|+\sqrt{-t}} \text { for all }(x, t) \in\left(\mathbb{R}^{3} \times(-\infty, 0]\right) \backslash\{(0,0)\} .
$$

Integration of this then immediately gives the upper bound of (12), which in fact holds true for $t \in(-1,0)$. Next, note that (66) implies

$$
\|u\|_{L_{t}^{\infty} L_{x}^{3, \infty}\left(\mathbb{R}^{3} \times(-\infty, 0)\right)} \leq M .
$$

We also remark that since $u$ is non-zero and $\lambda$-DSS we must have

$$
u \notin L_{x, t}^{\infty}\left(Q_{(0,0)}(r)\right) \text { for all sufficiently small } r .
$$

Indeed, suppose for contradiction that $u \in L_{x, t}^{\infty}\left(Q_{(0,0)}(r)\right)$ then for any $(x, t) \in \mathbb{R}^{3} \times$ $(-\infty, 0)$ we have $\left(\lambda^{-k} x, \lambda^{-2 k} t\right) \in Q_{(0,0)}(r)$ for all sufficiently large $k$. Using that $u$ is $\lambda$-DSS we have

$$
|u(x, t)|=\left|\lambda^{-k} u\left(\lambda^{-k} x, \lambda^{-2 k} t\right)\right| \leq \lambda^{-k}\|u\|_{L^{\infty}\left(Q_{(0,0)}(r)\right)} \downarrow 0 .
$$


From this point onward we fix

$$
t \in(-\infty, 0) \text { and } R \in\left(2 \sqrt{\frac{-t}{S_{B P}(M)}}, \infty\right) .
$$

Here, $S_{B P}(M)$ is as in Theorem A. Our aim is to show that with these choices (66)-(68) imply (12). First, given the fixed choices of $R$ and $t$ above, we take any large $T^{*}$ such that

$$
T^{*}>-2 t
$$

and

$$
R \in\left(2 \sqrt{\frac{-t}{S_{B P}(M)}}, e^{M^{1022}} \sqrt{T^{*}}\right) .
$$

Let us now consider the translated solution in time $u_{T^{*}}: \mathbb{R}^{3} \times\left[0, T^{*}\right] \rightarrow \mathbb{R}^{3}$ defined by

$$
u_{T^{*}}(x, s):=u\left(x, s-T^{*}\right) .
$$

Then (67)-(68) imply that

$$
\left\|u_{T^{*}}\right\|_{L_{t}^{\infty} L_{x}^{3, \infty}\left(\mathbb{R}^{3} \times\left(0, T^{*}\right)\right)} \leq M
$$

and

$$
u_{T^{*}} \notin L_{x, t}^{\infty}\left(Q_{\left(0, T^{*}\right)}(r)\right) \text { for all sufficiently small } r .
$$

Furthermore, (69)-(70) imply that for $s:=T^{*}+t$ we have

$$
\begin{aligned}
& s \in\left(\frac{T^{*}}{2}, T^{*}\right) \text { and } \\
& R \in\left(2 \sqrt{\frac{T^{*}-s}{S_{B P}(M)}}, e^{M^{1022}} \sqrt{T^{*}}\right) .
\end{aligned}
$$

The above allows us to apply Theorem A to $u_{T^{*}}$ to obtain

$$
\int_{|x|<R}\left|u_{T^{*}}(x, s)\right|^{3} d x \geq \frac{\log \left(\frac{R^{2}}{M^{802}\left|T^{*}-s\right|}\right)}{\exp \left(\exp \left(M^{1025}\right)\right)} .
$$

This then reduces to the desired lower bound in (12). The upper bound in (12) trivially follows from directly integrating (66).

Proof of Theorem B. Applying Proposition 2.2 we see that for $j=\left\lceil\exp \left(\exp \left(\left(M^{b}\right)^{1224}\right)\right)\right\rceil+$ 1 we have the contrapositive of (55). In particular,

$$
\left.\int_{B_{0}\left(4 \sqrt{S^{b}}-1\right.}\left|\omega\left(x, t_{(j+1)}\right)\right|^{2} d x<M^{2}\left(-t_{(j+1)}\right)^{\left.\frac{1}{2}\right)}\right)
$$

Almost identical arguments to those utilized in the proof of Lemma 3.1, except using the bound (177) instead of (178), give

$$
\|u\|_{L^{\infty}\left(B_{0}\left(C_{2} M^{50}\left(-t_{(j+1)}\right)^{\frac{1}{2}}\right) \times\left(\frac{t_{(j+1)}}{4}, 0\right)\right)} \leq \frac{C_{1} M^{-23}}{\left(-t_{(j+1)}\right)^{\frac{1}{2}}} .
$$


Since all estimates are independent of the spatial point where (55) occurs, we conclude that

$$
\|u\|_{L^{\infty}\left(\mathbb{R}^{3} \times\left(\frac{t_{(j+1)}}{4}, 0\right)\right)} \leq \frac{C_{1} M^{-23}}{\left(-t_{(j+1)}\right)^{\frac{1}{2}}} .
$$

This concludes the proof of the theorem.

\section{Proofs of the Main Quantitative Estimates}

3.1. Backward propagation of concentration. Here we state two pivotal results. These are concerned with backward propagation of concentration in the Type I case and in the time slices case. Figure 1 illustrates Lemma 3.1 below.

Lemma 3.1 (backward propagation of concentration, Type I). There exist two universal constants $C^{\sharp} \in\left(0, \frac{1}{16}\right), M_{3} \in[1, \infty)$ such that the following holds. For all $M \in$ $\left[M_{3}, \infty\right)$, there exists $S^{\sharp}(M) \in\left(0, \frac{1}{4}\right]$, such that the following holds. Let $(u, p)$ be a 'smooth solution with sufficient decay'22 of the Navier-Stokes equations (1) in I = $[-1,0]$ satisfying the Type I bound (48). Assume that there exists $t_{0}^{\prime} \in[-1,0)$ such that $t_{0}^{\prime}$ is not too close to -1 in the sense

$$
0<-t_{0}^{\prime}<C^{\sharp} M^{-548}
$$

and such that the vorticity concentrates at time $t_{0}^{\prime}$ in the following sense

$$
\begin{aligned}
& \int\left|\omega\left(x, t_{0}^{\prime}\right)\right|^{2} d x>M^{2}\left(-t_{0}^{\prime}\right)^{-\frac{1}{2}} \sqrt{S^{\sharp}} . \\
& B_{0}\left(4 \sqrt{S^{\sharp}}-1\left(-t_{0}^{\prime}\right)^{\frac{1}{2}}\right)
\end{aligned}
$$

Then, the vorticity concentrates in the following sense

$$
\int_{B_{0}\left(4 \sqrt{S^{\sharp}}\left(-t_{0}^{\prime \prime}\right)^{\frac{1}{2}}\right)}\left|\omega\left(x, t_{0}^{\prime \prime}\right)\right|^{2} d x>M^{2}\left(-t_{0}^{\prime \prime}\right)^{-\frac{1}{2}} \sqrt{S^{\sharp}}
$$

at any well-separated backward time $t_{0}^{\prime \prime} \in\left[-1, t_{0}^{\prime}\right]$ such that

$$
\frac{-t_{0}^{\prime}}{-t_{0}^{\prime \prime}}<C^{\sharp} M^{-548} \text {. }
$$

Here $S^{\sharp}(M)$ is defined explicitly by (76) and we have $S^{\sharp}(M)=C M^{-100}$.

Proof of Lemma 3.1. The proof is by contraposition. It relies on Theorem 5.1 below about local-in-space short-time smoothing. We define $S^{\sharp} \in\left(0, \frac{1}{4}\right]$ in the following way:

$$
S^{\sharp}=S^{\sharp}(M):=S_{*}\left(C_{\text {weak }} M, 32 C_{\text {Sob }}\left(1+C_{\text {ellip }}\right) C_{\text {weak }} M\right),
$$

22 See Sect. 1.4. 
where $S_{*}$ is the function defined in Theorem 5.1 (see also the formula (209)), $C_{\text {Sob }} \in$ $(0, \infty)$ is the best constant in the Sobolev embedding $H^{1}\left(B_{0}(2)\right) \subset L^{6}\left(B_{0}(2)\right)$ and $C_{\text {ellip }} \in(0, \infty)$ is the best constant in the estimate

$$
\|\nabla U(\cdot, 0)\|_{L^{2}\left(B_{0}(2)\right)} \leq C_{\text {ellip }}\left(\|\Omega(\cdot, 0)\|_{L^{2}\left(B_{0}(4)\right)}+\|U(\cdot, 0)\|_{L^{2}\left(B_{0}(4)\right)}\right)
$$

for weak solutions to

$$
-\Delta U(\cdot, 0)=\nabla \times \Omega(\cdot, 0) \text { in } B_{0}(4) .
$$

Furthermore, $C_{\text {weak }} \in[1, \infty)$ is a universal constant from the embedding $L^{3, \infty}\left(\mathbb{R}^{3}\right) \subset$ $L_{\text {uloc }}^{2}\left(\mathbb{R}^{3}\right)$. See, for example, Lemma 6.2 in Bradshaw and Tsai's paper [8].

Assume that

$$
\begin{aligned}
& \int\left|\omega\left(x, t_{0}^{\prime \prime}\right)\right|^{2} d x \leq M^{2}\left(-t_{0}^{\prime \prime}\right)^{-\frac{1}{2}} \sqrt{S^{\sharp}} \\
& B_{0}\left(4{\sqrt{S \sharp^{-1}}}^{-1}\left(-t_{0}^{\prime \prime}\right)^{\frac{1}{2}}\right)
\end{aligned}
$$

at a given time $t_{0}^{\prime \prime} \in[-1,0)$. Let $r:={\sqrt{S^{\sharp}}}^{-1}\left(-t_{0}^{\prime \prime}\right)^{\frac{1}{2}}$ and rescale in the following way $U(y, s):=r u\left(r y, r^{2} s+t_{0}^{\prime \prime}\right), \Omega(y, s):=r^{2} \omega\left(r y, r^{2} s+t_{0}^{\prime \prime}\right)$. We have

$$
\begin{aligned}
\|U(\cdot, 0)\|_{L^{6}\left(B_{0}(2)\right)} & \leq C_{S o b}\left(\|U(\cdot, 0)\|_{L^{2}\left(B_{0}(2)\right)}+\|\nabla U(\cdot, 0)\|_{L^{2}\left(B_{0}(2)\right)}\right) \\
& \leq C_{S o b}\left(1+C_{\text {ellip }}\right)\left(\|U(\cdot, 0)\|_{L^{2}\left(B_{0}(4)\right)}+\|\Omega(\cdot, 0)\|_{L^{2}\left(B_{0}(4)\right)}\right) \\
& \leq C_{S o b}\left(1+C_{\text {ellip }}\right)\left(8^{\frac{3}{2}}+1\right) C_{\text {weak }} M \\
& \leq 32 C_{S o b}\left(1+C_{\text {ellip }}\right) C_{\text {weak }} M .
\end{aligned}
$$

Here we used the scale-invariant bound (48) and the embedding $L^{3, \infty}\left(\mathbb{R}^{3}\right) \subset L_{\text {uloc }}^{2}\left(\mathbb{R}^{3}\right)$. Taking $M \geq M_{3}$ sufficiently large, we now apply the bound (178) with $C_{\text {weak }} M$ and $N:=32 C_{\text {Sob }}\left(1+C_{\text {ellip }}\right) C_{\text {weak }} M$. Then for any well-separated time $t_{0}^{\prime}$ satisfying (75), we have using $C^{\sharp} \in\left(0, \frac{1}{16}\right)$ and (75), that $-t_{0}^{\prime}<\frac{1}{16}\left(-t_{0}^{\prime \prime}\right)$. Therefore, we have $t_{0}^{\prime} \in$ $\left(t_{0}^{\prime \prime}+\frac{15}{16} S^{\sharp} r^{2}, t_{0}^{\prime \prime}+S^{\sharp} r^{2}\right)$, hence

$$
\begin{aligned}
\int_{B_{0}\left(4 \sqrt{S^{\sharp}}{ }^{-1}\left(-t_{0}^{\prime}\right)^{\frac{1}{2}}\right)}\left|\omega\left(x, t_{0}^{\prime}\right)\right|^{2} d x & \leq \sup _{t \in\left(t_{0}^{\prime \prime}+\frac{15}{16} S^{\sharp} r^{2}, t_{0}^{\prime \prime}+S^{\sharp} r^{2}\right)} \int_{B_{0}\left(4 \sqrt{S^{\sharp}}-1\right.} \int_{\left.\left(-t_{0}^{\prime}\right)^{\frac{1}{2}}\right)}|\omega(x, t)|^{2} d x \\
& \leq \sup _{t \in\left(t_{0}^{\prime \prime}+\frac{15}{16} S^{\sharp} r^{2}, t_{0}^{\prime \prime}+S^{\sharp} r^{2}\right)} \int_{B_{0}\left(\frac{1}{4} \sqrt{S^{\sharp}}\right.}|\omega(x, t)|^{2} d x \\
& \leq r^{-1} \sup _{s \in\left(\frac{15}{16} S^{\sharp}, S^{\sharp}\right)} \int_{B_{0}\left(\frac{1}{4}\right)}|\Omega(y, s)|^{2} d x \\
& \leq C M^{65} N^{161}\left(-t_{0}^{\prime \prime}\right)^{-\frac{1}{2}} \\
& \leq C M^{226}\left(-t_{0}^{\prime \prime}\right)^{-\frac{1}{2}} .
\end{aligned}
$$

The conclusion follows then from (75) for a well chosen universal constant $C^{\sharp} \in\left(0, \frac{1}{16}\right)$. 
A variant of the proof of Lemma 3.1 gives the following concentration result for the enstrophy near a Type I singularity.

Lemma 3.2 (Concentration of the enstrophy). For all sufficiently large $M \in[1, \infty)$, let $S^{\sharp}(M) \in\left(0, \frac{1}{4}\right]$ be the constant defined by $(76)$. Let $(u, p)$ be a suitable finiteenergy solution ${ }^{23}$ of the Navier-Stokes equations (1) in $I=[-1,0]$ satisfying the Type I bound (48). Assume that the space-time point $(0,0)$ is a singularity for $u$. Then, for all $t^{\prime} \in \Sigma$, where $\Sigma$ is a full measure subset of $[-1,0)$ defined in Sect. 1.4.4, the vorticity concentrates in the following sense

$$
\begin{aligned}
& \int\left|\omega\left(x, t^{\prime}\right)\right|^{2} d x>M^{2}\left(-t^{\prime}\right)^{-\frac{1}{2}} \sqrt{S^{\sharp}} . \\
& B_{0}\left(4{\sqrt{S^{\sharp}}}^{-1}\left(-t^{\prime}\right)^{\frac{1}{2}}\right)
\end{aligned}
$$

Proof of Lemma 3.2. Indeed, if (79) does not hold, one takes $r={\sqrt{S^{\sharp}}}^{-1}\left(-t^{\prime}\right)^{\frac{1}{2}}$ and performs the rescaling $U(y, s)=r u\left(r y, r^{2} s+t^{\prime}\right), \Omega(y, s)=r^{2} \omega\left(r y, r^{2} s+t^{\prime}\right)$. The same reasoning as in Lemma 3.1 gives that we can apply the bound (177) with $C_{\text {weak }} M$ and $N=32 C_{S o b}\left(1+C_{\text {ellip }}\right) C_{\text {weak }} M$ (see also (78)). This gives

$$
\|u\|_{L^{\infty}\left(B_{0}\left(\frac{1}{2} \sqrt{S^{\sharp}}\left(-t^{\prime}\right)^{\frac{1}{2}}\right) \times\left(\frac{1}{4} t^{\prime}, 0\right)\right)} \leq \frac{C_{*} M^{8} N^{19} \sqrt{S^{\sharp}}}{\left(-t^{\prime}\right)^{\frac{1}{2}}} \leq \frac{C M^{-23}}{\left(-t^{\prime}\right)^{\frac{1}{2}}} .
$$

This contradicts the assumption that $(0,0)$ is a singular point of $u$.

Lemma 3.3 (backward propagation of concentration, time slices). There exists a universal constant $M_{4} \in[1, \infty)$ such that the following holds true. Let $M \in\left[M_{4}, \infty\right)$. We define $M^{b}$ by (17). Fix any $\alpha \geq M^{b}$ and let $t_{0}^{\prime}, t_{0}^{\prime \prime} \in[-1,0)$ be such that

$$
\frac{t_{0}^{\prime \prime}}{\alpha^{1051}}<t_{0}^{\prime}<0
$$

There exists $S^{b}(M) \in\left(0, \frac{1}{4}\right]$, such that the following holds. Let $(u, p)$ be a $C^{\infty}\left(\mathbb{R}^{3} \times\right.$ $(-1,0))$ finite-energy solution of the Navier-Stokes equations $(1)$ in $I=[-1,0]$ satisfying

$$
\left\|u\left(\cdot, t_{0}^{\prime}\right)\right\|_{L^{3}} \leq M \text { and }\left\|u\left(\cdot, t_{0}^{\prime \prime}\right)\right\|_{L^{3}} \leq M .
$$

Suppose further that the vorticity concentrates at time $t_{0}^{\prime}$ in the following sense

$$
\int_{B_{0}\left(4 \sqrt{S^{b^{-1}}}\left(-t_{0}^{\prime}\right)^{\frac{1}{2}}\right)}\left|\omega\left(x, t_{0}^{\prime}\right)\right|^{2} d x>M^{2}\left(-t_{0}^{\prime}\right)^{-\frac{1}{2}} \sqrt{S^{b}} .
$$

The above assumptions imply that for any $s_{0} \in\left[t_{0}^{\prime \prime}, \frac{t_{0}^{\prime \prime}}{8 \alpha^{201}}\right]$ the vorticity concentrates in the following sense

$$
\int_{B_{0}\left(4\left(-s_{0}\right)^{\frac{1}{2}} \alpha^{106}\right)}\left|\omega\left(x, s_{0}\right)\right|^{2} d x>\frac{(M+1)^{2}}{\left(-s_{0}\right)^{\frac{1}{2}} \alpha^{106}} .
$$

Here $S^{b}(M)=C M^{-100}$.

23 See Sect. 1.4. 
Proof. The proof below uses Theorem 5.1 and Remark 5.2. For $s \in\left[t_{0}^{\prime \prime}, 0\right]$ we decompose $u$ as

$$
u(\cdot, s)=e^{\left(s-t_{0}^{\prime \prime}\right) \Delta} u\left(\cdot, t_{0}^{\prime \prime}\right)+V(\cdot, s)
$$

We then have

$$
\begin{aligned}
\left\|e^{\left(s-t_{0}^{\prime \prime}\right) \Delta} u\left(\cdot, t_{0}^{\prime \prime}\right)\right\|_{L_{x}^{3}} & \leq M . \\
\left\|e^{\left(s-t_{0}^{\prime \prime}\right) \Delta} u\left(\cdot, t_{0}^{\prime \prime}\right)\right\|_{L_{x}^{4}} & \leq \frac{C M}{\left(s_{0}-t_{0}^{\prime \prime}\right)^{\frac{1}{8}}} .
\end{aligned}
$$

Furthermore, arguments from [17] imply that

$$
\left\|e^{\left(t-t_{0}^{\prime \prime}\right) \Delta} u\left(\cdot, t_{0}^{\prime \prime}\right)\right\|_{L^{5}\left(\mathbb{R}^{3} \times\left(t_{0}^{\prime \prime}, \infty\right)\right)} \leq C M
$$

Moreover, similar arguments as those used in Proposition 2.2 of [40] ${ }^{24}$ yield that for $s \in\left[t_{0}^{\prime \prime}, 0\right]$

$$
\begin{aligned}
& \|V(\cdot, s)\|_{L_{x}^{2}}^{2}+\int_{t_{0}^{\prime \prime}}^{s} \int_{\mathbb{R}^{3}}|\nabla V|^{2} d x d t \\
& \quad \leq C \int_{t_{0}^{\prime \prime}}^{s} \int_{\mathbb{R}^{3}}\left|e^{\left(t-t_{0}^{\prime \prime}\right) \Delta} u\left(\cdot, t_{0}^{\prime \prime}\right)\right|^{4} d x d t+C \int_{t_{0}^{\prime \prime}}^{s}\|V(\cdot, t)\|_{L_{x}^{2}}^{2}\left\|e^{\left(t-t_{0}^{\prime \prime}\right) \Delta} u\left(\cdot, t_{0}^{\prime \prime}\right)\right\|_{L_{x}^{5}}^{5} d t .
\end{aligned}
$$

Using (85)-(87) and Gronwall's lemma, we infer (for $M$ larger than some universal constant) that

$$
\|V(\cdot, s)\|_{L_{x}^{2}}^{2}+\int_{t_{0}^{\prime \prime}}^{s} \int_{\mathbb{R}^{3}}|\nabla V(x, t)|^{2} d x d t \leq C\left(M^{\mathrm{b}}\right)^{4}\left(s-t_{0}^{\prime \prime}\right)^{\frac{1}{2}} .
$$

Here $M^{b}$ is defined by (17) for an appropriate universal constant $L_{*} \in(0, \infty)$ coming from the Gronwall estimate. In particular, using that $s \in\left[t_{0}^{\prime \prime}, \frac{t_{0}^{\prime \prime}}{8 \alpha^{201}}\right]$ we have

$$
\|V(\cdot, s)\|_{L_{x}^{2}}^{2} \leq C 8^{\frac{1}{2}} \alpha^{101}\left(M^{b}\right)^{4}(-s)^{\frac{1}{2}}<\alpha^{106}(-s)^{\frac{1}{2}} .
$$

Here, we used the fact that $\alpha \geq M^{b}$.

From now on the proof is by contraposition. We assume that for a given $t_{0}^{\prime \prime} \in[-1,0)$ there exists $s_{0} \in\left[t_{0}^{\prime \prime}, \frac{t_{0}^{\prime \prime}}{8 \alpha^{201}}\right]$ such that

$$
\int_{B_{0}\left(4 \alpha^{106}\left(-s_{0}\right)^{\frac{1}{2}}\right)}\left|\omega\left(x, s_{0}\right)\right|^{2} d x \leq \frac{(M+1)^{2}}{\left(-s_{0}\right)^{\frac{1}{2}} \alpha^{106}} .
$$

Define

$$
\lambda:=\left(-s_{0}\right)^{\frac{1}{2}} \alpha^{106}
$$

\footnotetext{
24 Based on the energy method, Sobolev embedding, Hölder's inequality and Young's inequality.
} 
and rescale to get $U^{\lambda}: \mathbb{R}^{3} \times\left(0, \alpha^{-212}\right) \rightarrow \mathbb{R}^{3}$ and $P^{\lambda}: \mathbb{R}^{3} \times\left(0, \alpha^{-212}\right) \rightarrow \mathbb{R}$. Here,

$$
U^{\lambda}(y, t):=\lambda u\left(\lambda y, \lambda^{2} t+s_{0}\right) \text { and } P^{\lambda}(y, t):=\lambda^{2} p\left(\lambda y, \lambda^{2} t+s_{0}\right) .
$$

Using (85) and (88), we see that

$$
\left\|U^{\lambda}(y, 0)\right\|_{L_{u l o c}^{2}} \leq C_{L e b} M+1 .
$$

Here, $C_{\text {Leb }} \in[1, \infty)$ is a universal constant from the embedding $L^{3}\left(\mathbb{R}^{3}\right) \subset L_{u l o c}^{2}\left(\mathbb{R}^{3}\right)$.

Furthermore, defining $\Omega^{\lambda}=\nabla \times U^{\lambda}$, we see that (89) implies that

$$
\int_{B_{0}(4)}\left|\Omega^{\lambda}(y, 0)\right|^{2} d y \leq(M+1)^{2} .
$$

Similarly to Lemma 3.1, we define

$$
S^{b}=S^{b}(M):=S_{*}\left(C_{L e b} M+1,32 C_{S o b}\left(1+C_{\text {ellip }}\right)\left(C_{L e b} M+1\right)\right)
$$

where $S_{*}$ is the constant defined in Theorem 5.1, $C_{S o b} \in(0, \infty)$ is the best constant in the Sobolev embedding $H^{1}\left(B_{0}(2)\right) \subset L^{6}\left(B_{0}(2)\right)$ and $C_{\text {ellip }} \in(0, \infty)$ is the best constant in the estimate

$$
\|\nabla U(\cdot, 0)\|_{L^{2}\left(B_{0}(2)\right)} \leq C_{\text {ellip }}\left(\|\Omega(\cdot, 0)\|_{L^{2}\left(B_{0}(4)\right)}+\|U(\cdot, 0)\|_{L^{2}\left(B_{0}(4)\right)}\right)
$$

for weak solutions to

$$
-\Delta U(\cdot, 0)=\nabla \times \Omega(\cdot, 0) \text { in } B_{0}(4) .
$$

Next notice that from (17), if we have for $M$ sufficiently large

$$
\alpha^{-212}<\left(M^{b}\right)^{-212}<(1+M)^{-101}<S^{b} .
$$

Using (92)-(93), together with a similar reasoning to Lemma 3.1, we can apply Theorem 5.1 with $M \geq M_{4}$ being sufficiently large. Specifically, we apply Remark 5.2 with $\beta=\alpha^{-212}$. This gives

$$
\begin{aligned}
& \left\|\nabla U^{\lambda}\right\|_{L_{t}^{\infty} L_{x}^{2}\left(B_{0}\left(\frac{1}{6}\right) \times\left(\frac{255}{256} \alpha^{-212}, \alpha^{-212}\right)\right)} \\
& \quad \leq C_{\text {univ }} M^{4} \alpha^{265} .
\end{aligned}
$$

This implies that

$$
\begin{aligned}
& \|\nabla u\|^{2} L_{t}^{\infty} L_{x}^{2}\left(B_{0}\left(\frac{\left(-s_{0}\right)^{\frac{1}{2}} \alpha^{106}}{6}\right) \times\left(\frac{s_{0}}{256}, 0\right)\right) \\
& \leq \frac{C_{u n i v}^{2} M^{8} \alpha^{424}}{\left(-s_{0}\right)^{\frac{1}{2}}} .
\end{aligned}
$$

Using that $s_{0}<t_{0}^{\prime}<0$ and that $S^{b}(M)=C M^{-100}$, we have for $M$ sufficiently large that

$$
B_{0}\left(4{\sqrt{S^{b}}}^{-1}\left(-t_{0}^{\prime}\right)^{\frac{1}{2}}\right) \subset B_{0}\left(\frac{1}{6}\left(-s_{0}\right)^{\frac{1}{2}}\left(M^{b}\right)^{106}\right) .
$$


So for $\frac{s_{0}}{256}<t_{0}^{\prime}$, we see that (95) implies that

$$
\left.\int_{B_{0}\left(4 \sqrt{S^{b}}-1\right.} \mid \omega\left(x, t_{0}^{\prime}\right)^{\frac{1}{2}}\right)\left.\right|^{2} d x \leq \frac{C_{\text {univ }}^{2} M^{8} \alpha^{424}}{\left(-s_{0}\right)^{\frac{1}{2}}} \leq \frac{M^{8} \alpha^{525}}{\left(-t_{0}^{\prime \prime}\right)^{\frac{1}{2}}} .
$$

Here, we used $s_{0} \in\left[t_{0}^{\prime \prime}, \frac{t_{0}^{\prime \prime}}{8 \alpha^{201}}\right]$. Thus,

$$
\int_{B_{0}\left(4 \sqrt{S^{b^{-1}}}\left(-t_{0}^{\prime}\right)^{\frac{1}{2}}\right)}\left|\omega\left(x, t_{0}^{\prime}\right)\right|^{2} d x \leq M^{2}\left(-t_{0}^{\prime}\right)^{-\frac{1}{2}} \sqrt{S^{b}} \times \frac{\alpha^{525} M^{6}}{\sqrt{S^{b}}}\left(\frac{-t_{0}^{\prime}}{-t_{0}^{\prime \prime}}\right)^{\frac{1}{2}} .
$$

Now,

$$
\frac{\alpha^{525} M^{6}}{\sqrt{S^{b}}}\left(\frac{-t_{0}^{\prime}}{-t_{0}^{\prime \prime}}\right)^{\frac{1}{2}} \leq C_{\text {univ }} M^{56} \alpha^{525}\left(\frac{-t_{0}^{\prime}}{-t_{0}^{\prime \prime}}\right)^{\frac{1}{2}} .
$$

Therefore, since (80) holds, we get the conclusion.

3.2. Proof of the main quantitative estimate in the Type I case. This part is devoted to the proof of Proposition 2.1. The proof of Proposition 2.1 uses Lemma 3.1, Corollary 6.7, Lemma 7.3 and the Carleman inequalities in "Appendix B". Following Tao [47], the idea of the proof is to transfer the concentration of the enstrophy at times $t_{0}^{\prime \prime}$ far away in the past to large-scale lower bounds for the enstrophy at time $t_{0}$. This is done in Step 1-3 below. The last step, Step 4 below, consists in transferring the lower bound on the enstrophy at time $t_{0}$ to a lower bound for the $L^{3}$ norm at time $t_{0}$ and summing appropriate scales. The assumption (49) at the final time $t_{0}$ is critical.

Without loss of generality, we now take $t_{0}=0$. We also assume that $T=1$. The general statement is obtained by scaling. Let $M \in\left[M_{3}, \infty\right)$ where $M_{3}$ is a constant in Lemma 3.1. In the course of the proof we will need to take $M$ larger, always larger than universal constants. Let $u: \mathbb{R}^{3} \times[-1,0] \rightarrow \mathbb{R}^{3}$ be a 'smooth solution with sufficient decay' ${ }^{25}$ of the Navier-Stokes equations (1) in $I=[-1,0]$ satisfying the Type I bound (48). Assume that there exists $t_{0}^{\prime} \in[-1,0)$ such that $t_{0}^{\prime}$ is not too close to -1 in the sense $^{26}$

$$
0<-t_{0}^{\prime}<\frac{C^{\sharp}}{8} M^{-749}
$$

and such that the vorticity concentrates at time $t_{0}^{\prime}$ in the following sense

$$
\begin{aligned}
& \int\left|\omega\left(x, t_{0}^{\prime}\right)\right|^{2} d x>M^{2}\left(-t_{0}^{\prime}\right)^{-\frac{1}{2}} \sqrt{S^{\sharp}}, \\
& B_{0}\left(4{\sqrt{S^{\sharp}}}^{-1}\left(-t_{0}^{\prime}\right)^{\frac{1}{2}}\right)
\end{aligned}
$$

where we recall that $S^{\sharp}=C M^{-100}$. Lemma 3.1 then implies that

$$
\begin{aligned}
& \int\left|\omega\left(x, t^{\prime \prime}\right)\right|^{2} d x>M^{2}\left(-t^{\prime \prime}\right)^{-\frac{1}{2}} \sqrt{S^{\sharp}} . \\
& B_{0}\left(4{\sqrt{S^{\sharp}}}^{-1}\left(-t^{\prime \prime}\right)^{\frac{1}{2}}\right)
\end{aligned}
$$

25 See Sect. 1.4.

26 The number $M^{-749}$ in the right hand side is required by (106). 
at any well-separated backward time $t^{\prime \prime} \in\left[-1, t_{0}^{\prime}\right]$ such that ${ }^{27}$

$$
\frac{1}{C^{\sharp}} M^{548} t_{0}^{\prime}>t^{\prime \prime} .
$$

The rest of the proof relies on the Carleman inequalities of Proposition B.1 and Proposition B.2. These are the tools used to transfer the concentration information (97) from the time $t^{\prime \prime}$ to time 0 and from the small scales $B_{0}\left(4 \sqrt{S^{\sharp}}-1\left(-t^{\prime \prime}\right)^{\frac{1}{2}}\right)$ to large scales.

Step 1: quantitative unique continuation. The purpose of this step is to prove the following estimate:

$$
T_{1}^{\frac{1}{2}} e^{-\frac{C M^{149} R^{2}}{T_{1}}} \lesssim \int_{-T_{1}}^{-\frac{T_{1}}{2}} \int_{B_{0}(2 R) \backslash B_{0}(R / 2)}|\omega(x, t)|^{2} d x d t,
$$

for all $T_{1}$ and $R$ such that

$$
\frac{2}{C^{\sharp}} M^{548}\left(-t_{0}^{\prime}\right)<T_{1} \leq \frac{1}{2} \text { and } R \geq M^{100}\left(\frac{T_{1}}{2}\right)^{\frac{1}{2}} .
$$

Let $t_{0}^{\prime \prime} \geq-\frac{1}{2}$ be such that (98) is satisfied with $t^{\prime \prime}=\frac{t_{0}^{\prime \prime}}{2}$. Let $T_{1}:=-t_{0}^{\prime \prime}$ and $I_{1}:=$ $\left(t_{0}^{\prime \prime}, t_{0}^{\prime \prime}+\frac{T_{1}}{2}\right)=\left(-T_{1},-\frac{T_{1}}{2}\right) \subset\left[-\frac{1}{2}, 0\right] \subset[-1,0]$. Thus, we can apply Lemma 7.3 and Remark 7.4 with $t_{0}=0$ and $T=1$. The bound (259) in Remark 7.4 implies that there exists an epoch of regularity $I_{1}^{\prime \prime}=\left[t_{1}^{\prime \prime}-T_{1}^{\prime \prime}, t_{1}^{\prime \prime}\right] \subset I_{1}$ such that

$$
T_{1}^{\prime \prime}=\left|I_{1}^{\prime \prime}\right|=\frac{M^{-48}}{4 C_{\text {univ }}^{3}}\left|I_{1}\right|=\frac{M^{-48}}{8 C_{\text {univ }}^{3}} T_{1}
$$

and for $j=0,1,2$,

$$
\left\|\nabla^{j} u\right\|_{L_{t}^{\infty} L_{x}^{\infty}\left(\mathbb{R}^{3} \times I_{1}^{\prime \prime}\right)} \leq \frac{1}{2^{j+1}}\left|I_{1}^{\prime \prime}\right|^{\frac{-(j+1)}{2}}=\frac{1}{2^{j+1}}\left(T_{1}^{\prime \prime}\right)^{\frac{-(j+1)}{2}} .
$$

Let $T_{1}^{\prime \prime \prime}:=\frac{3}{4} T_{1}^{\prime \prime}$ and $s^{\prime \prime} \in\left[t_{1}^{\prime \prime}-\frac{T_{1}^{\prime \prime}}{4}, t_{1}^{\prime \prime}\right]$. Let $x_{1} \in \mathbb{R}^{3}$ be such that $\left|x_{1}\right| \geq M^{100}\left(\frac{T_{1}}{2}\right)^{\frac{1}{2}}$ and let $r_{1}:=M^{50}\left|x_{1}\right| \geq M^{150}\left(\frac{T_{1}}{2}\right)^{\frac{1}{2}}$. Notice that for $M$ large enough

$$
r_{1}:=M^{50}\left|x_{1}\right| \geq M^{150}\left(\frac{T_{1}}{2}\right)^{\frac{1}{2}} \geq M^{99} \cdot 4{\sqrt{S^{\sharp}}}^{-1}\left(-t_{0}^{\prime \prime}\right)^{\frac{1}{2}}
$$

and

$$
r_{1}^{2} \geq 4000 T_{1}^{\prime \prime \prime}
$$

We apply the second Carleman inequality, Proposition B.2 (quantitative unique continuation), on the cylinder $\mathcal{C}_{1}=\left\{(x, t) \in \mathbb{R}^{3} \times \mathbb{R}: t \in\left[0, T_{1}^{\prime \prime \prime}\right],|x| \leq r_{1}\right\}$ to the function $w: \mathbb{R}^{3} \times\left[0, T_{1}^{\prime \prime \prime}\right] \rightarrow \mathbb{R}^{3}$, defined by for all $(x, t) \in \mathbb{R}^{3} \times\left[0, T_{1}^{\prime \prime \prime}\right]$,

$$
w(x, t):=\omega\left(x_{1}+x, s^{\prime \prime}-t\right) .
$$

27 Notice that the whole argument of Sect. 3.2 goes through assuming that (97) holds for almost any $t^{\prime \prime} \in\left[-1, \frac{1}{C^{\sharp}} M^{548} t_{0}^{\prime}\right)$. 
Notice that the quantitative regularity (102) and the vorticity equation (37) imply that on $\mathcal{C}_{1}$

$$
\left|\left(\partial_{t}+\Delta\right) w\right| \leq \frac{3}{16} T_{1}^{\prime \prime \prime}-1|w|+\frac{\sqrt{3}}{4} T_{1}^{\prime \prime \prime}-\frac{1}{2}|\nabla w|,
$$

so that (310) is satisfied with $S=S_{1}:=T_{1}^{\prime \prime \prime}$ and $C_{\text {Carl }}=\frac{16}{3}$. Let

$$
\bar{s}_{1}=\frac{T_{1}^{\prime \prime \prime}}{20000}, \quad \underline{s}_{1}=M^{-150} T_{1}^{\prime \prime \prime}
$$

For $M$ sufficiently large we have $0<\underline{s}_{1} \leq \bar{s}_{1} \leq \frac{T_{1}^{\prime \prime \prime}}{10000}$. Hence by (312) we have

$$
Z_{1} \lesssim e^{-\frac{r_{1}^{2}}{500 \bar{s}_{1}}} X_{1}+\left(\bar{s}_{1}\right)^{\frac{3}{2}}\left(\frac{e \bar{s}_{1}}{\underline{s}_{1}}\right)^{\frac{C r_{1}^{2}}{\bar{s}_{1}}} Y_{1},
$$

where

$$
\begin{aligned}
X_{1}:= & \int_{s^{\prime \prime}-T_{1}^{\prime \prime \prime}} \int_{B_{x_{1}}\left(M^{50}\left|x_{1}\right|\right)}\left(\left(T_{1}^{\prime \prime \prime}\right)^{-1}|\omega|^{2}+|\nabla \omega|^{2}\right) d x d s, \\
Y_{1}:= & \int_{B_{x_{1}}\left(M^{50}\left|x_{1}\right|\right)}\left|\omega\left(x, s^{\prime \prime}\right)\right|^{2}\left(\underline{s}_{1}\right)^{-\frac{3}{2}} e^{-\frac{\left|x-x_{1}\right|^{2}}{4 \underline{s}_{1}}} d x, \\
Z_{1}:= & \int_{s^{\prime \prime}-\frac{T_{1}^{\prime \prime \prime}}{20000}} \int_{s^{\prime \prime}-\frac{T_{1}^{\prime \prime \prime}}{10000} B_{x_{1}}\left(\frac{M^{50}\left|x_{1}\right|}{2}\right)}\left(\left(T_{1}^{\prime \prime \prime}\right)^{-1}|\omega|^{2}+|\nabla \omega|^{2}\right) e^{-\frac{\left|x-x_{1}\right|^{2}}{4\left(s^{\prime \prime}-s\right)}} d x d s .
\end{aligned}
$$

We first use the concentration (97) for times $s \in\left[s^{\prime \prime}-\frac{T_{1}^{\prime \prime \prime}}{10000}, s^{\prime \prime}-\frac{T_{1}^{\prime \prime \prime}}{20000}\right]$ to bound $Z_{1}$ from below. By (103), we have

$$
B_{0}\left(4{\sqrt{S^{\sharp}}}^{-1}(-s)^{\frac{1}{2}}\right) \subset B_{x_{1}}\left(2\left|x_{1}\right|\right) \subset B_{x_{1}}\left(\frac{M^{50}\left|x_{1}\right|}{2}\right)
$$

for all $s \in\left[s^{\prime \prime}-\frac{T_{1}^{\prime \prime \prime}}{10000}, s^{\prime \prime}-\frac{T_{1}^{\prime \prime \prime}}{20000}\right]$ and for $M$ sufficiently large. We have

$$
\begin{aligned}
& Z_{1} \gtrsim \int_{s^{\prime \prime}-\frac{T_{1}^{\prime \prime \prime}}{10000} B_{0}\left(4 \sqrt{S^{\sharp}}-1\right.}^{s^{\prime \prime}-\frac{T_{1}^{\prime \prime \prime}}{20000}} \int_{\left.(-s)^{\frac{1}{2}}\right)}\left(T_{1}^{\prime \prime \prime}\right)^{-1}|\omega(x, s)|^{2} d x d s e^{-\frac{C\left|x_{1}\right|^{2}}{T_{1}^{\prime \prime \prime}}} \\
& \gtrsim \int_{s^{\prime \prime}-\frac{T_{1}^{\prime \prime \prime}}{10000}}^{s^{\prime \prime}-\frac{T_{1}^{\prime \prime \prime}}{20000}} M^{-48}(-s)^{-\frac{1}{2}} d s\left(T_{1}^{\prime \prime}\right)^{-1} e^{-\frac{C\left|x_{1}\right|^{2}}{T_{1}^{\prime \prime}}}
\end{aligned}
$$




$$
\begin{aligned}
& \gtrsim M^{-48} \frac{T_{1}^{\prime \prime \prime}}{\left(-S^{\prime \prime}+\frac{T_{1}^{\prime \prime \prime}}{10000}\right)^{\frac{1}{2}}}\left(T_{1}^{\prime \prime}\right)^{-1} e^{-\frac{C\left|x_{1}\right|^{2}}{T_{1}^{\prime \prime}}} \\
& \gtrsim M^{-48}\left(T_{1}\right)^{-\frac{1}{2}} e^{-\frac{C\left|x_{1}\right|^{2}}{T_{1}^{\prime \prime}}} \\
& \gtrsim M^{-48}\left(M^{48} T_{1}^{\prime \prime}\right)^{-\frac{1}{2}} e^{-\frac{C\left|x_{1}\right|^{2}}{T_{1}^{\prime \prime}}} \\
& =M^{-72}\left(T_{1}^{\prime \prime}\right)^{-\frac{1}{2}} e^{-\frac{C\left|x_{1}\right|^{2}}{T_{1}^{\prime \prime}}} .
\end{aligned}
$$

Second, we bound from above $X_{1}$. We rely on the quantitative regularity (102) to obtain

$$
X_{1} \lesssim\left(T_{1}^{\prime \prime}\right)^{-2} M^{150}\left|x_{1}\right|^{3} .
$$

Hence,

$$
\begin{aligned}
e^{-\frac{r_{1}^{2}}{500 \bar{s}_{1}}} X_{1} & \lesssim\left(T_{1}^{\prime \prime}\right)^{-2} M^{150}\left|x_{1}\right|^{3} e^{-\frac{C M^{100}\left|x_{1}\right|^{2}}{T_{1}^{\prime \prime}}} \\
& \lesssim\left(T_{1}^{\prime \prime}\right)^{-\frac{1}{2}} e^{-\frac{C M^{100}\left|x_{1}\right|^{2}}{T_{1}^{\prime \prime}}} .
\end{aligned}
$$

Third, for $Y_{1}$ we decompose and estimate as follows

$$
\begin{aligned}
Y_{1}: & =\int_{B_{x_{1}}\left(\frac{\left|x_{1}\right|}{2}\right)}\left|\omega\left(x, s^{\prime \prime}\right)\right|^{2}\left(\underline{s}_{1}\right)^{-\frac{3}{2}} e^{-\frac{\left|x-x_{1}\right|^{2}}{4 s_{1}}} d x \\
& +\int_{B_{x_{1}}\left(M^{50}\left|x_{1}\right|\right) \backslash B_{x_{1}}\left(\frac{\left|x_{1}\right|}{2}\right)}\left|\omega\left(x, s^{\prime \prime}\right)\right|^{2}\left(\underline{s}_{1}\right)^{-\frac{3}{2}} e^{-\frac{\left|x-x_{1}\right|^{2}}{4 s_{1}}} d x \\
\lesssim & M^{225}\left(T_{1}^{\prime \prime}\right)^{-\frac{3}{2}}\left(\int_{B_{x_{1}}\left(\frac{\left|x_{1}\right|}{2}\right)}\left|\omega\left(x, s^{\prime \prime}\right)\right|^{2} d x\right. \\
& \left.+\int_{B_{x_{1}}\left(M^{50}\left|x_{1}\right|\right) \backslash B_{x_{1}}\left(\frac{\left|x_{1}\right|}{2}\right)}\left|\omega\left(x, s^{\prime \prime}\right)\right|^{2} e^{-\frac{C M^{150}\left|x_{1}\right|^{2}}{T_{1}^{\prime \prime}}} d x\right) \\
\lesssim & M^{225}\left(T_{1}^{\prime \prime}\right)^{-\frac{3}{2}}\left(\int_{B_{x_{1}}\left(\frac{\left|x_{1}\right|}{2}\right)}\left|\omega\left(x, s^{\prime \prime}\right)\right|^{2} d x+M^{150}\left|x_{1}\right|^{3}\left(T_{1}^{\prime \prime}\right)^{-2} e^{-\frac{C M^{150}\left|x_{1}\right|^{2}}{T_{1}^{\prime \prime}}}\right) \\
& \lesssim M^{225}\left(T_{1}^{\prime \prime}\right)^{-\frac{3}{2}}\left(\int_{B_{x_{1}}\left(\frac{\left|x_{1}\right|}{2}\right)}\left|\omega\left(x, s^{\prime \prime}\right)\right|^{2} d x+\left(T_{1}^{\prime \prime}\right)^{-\frac{1}{2}} e^{-\frac{C M^{150}\left|x_{1}\right|^{2}}{T_{1}^{\prime \prime}}}\right),
\end{aligned}
$$

where we used the quantitative regularity (102). Hence,

$$
\left(\bar{s}_{1}\right)^{\frac{3}{2}}\left(\frac{e \bar{s}_{1}}{\underline{s}_{1}}\right)^{\frac{C r_{1}^{2}}{\bar{s}_{1}}} Y_{1} \lesssim\left(T_{1}^{\prime \prime}\right)^{\frac{3}{2}} e^{\frac{C M^{100}\left|x_{1}\right|^{2}}{T_{1}^{\prime \prime}} \log \left(\frac{e M^{150}}{20000}\right)} Y_{1}
$$




$$
\begin{gathered}
\lesssim M^{225} e^{\frac{C M^{101}\left|x_{1}\right|^{2}}{T_{1}^{\prime \prime}}} \int_{B_{x_{1}}\left(\frac{\left|x_{1}\right|}{2}\right)}\left|\omega\left(x, s^{\prime \prime}\right)\right|^{2} d x \\
+M^{225}\left(T_{1}^{\prime \prime}\right)^{-\frac{1}{2}} e^{-\frac{C M^{150}\left|x_{1}\right|^{2}}{T_{1}^{\prime \prime}}} .
\end{gathered}
$$

Gathering these bounds and combining with (104) yields

$$
\begin{gathered}
M^{-72}\left(T_{1}^{\prime \prime}\right)^{-\frac{1}{2}} e^{-\frac{C\left|x_{1}\right|^{2}}{T_{1}^{\prime \prime}}} \lesssim\left(T_{1}^{\prime \prime}\right)^{-\frac{1}{2}} e^{-\frac{C M^{100}\left|x_{1}\right|^{2}}{T_{1}^{\prime \prime}}} \\
+M^{225} e^{\frac{C M^{101}\left|x_{1}\right|^{2}}{T_{1}^{\prime \prime}}} \int_{B_{x_{1}}\left(\frac{\left|x_{1}\right|}{2}\right)}\left|\omega\left(x, s^{\prime \prime}\right)\right|^{2} d x+M^{225}\left(T_{1}^{\prime \prime}\right)^{-\frac{1}{2}} e^{-\frac{C M^{150}\left|x_{1}\right|^{2}}{T_{1}^{\prime \prime}}} .
\end{gathered}
$$

Using (101) and $\left|x_{1}\right| \geq M^{100}\left(\frac{T_{1}}{2}\right)^{\frac{1}{2}}$, we see that for $M$ sufficiently large

$$
M^{-297}\left(T_{1}^{\prime \prime}\right)^{-\frac{1}{2}} e^{-\frac{C M^{101}\left|x_{1}\right|^{2}}{T_{1}^{\prime \prime}}} \lesssim \int_{B_{x_{1}}\left(\frac{\left|x_{1}\right|}{2}\right)}\left|\omega\left(x, s^{\prime \prime}\right)\right|^{2} d x
$$

Hence, for all $s^{\prime \prime} \in\left[t_{1}^{\prime \prime}-\frac{T_{1}^{\prime \prime}}{4}, t_{1}^{\prime \prime}\right]$, for all $\left|x_{1}\right| \geq M^{100}\left(\frac{T_{1}}{2}\right)^{\frac{1}{2}}$,

$$
\int_{B_{x_{1}}\left(\frac{\left|x_{1}\right|}{2}\right)}\left|\omega\left(x, s^{\prime \prime}\right)\right|^{2} d x \gtrsim M^{-297}\left(T_{1}^{\prime \prime}\right)^{-\frac{1}{2}} e^{-\frac{C M^{101}\left|x_{1}\right|^{2}}{T_{1}^{\prime \prime}}} .
$$

Let $R \geq M^{100}\left(\frac{T_{1}}{2}\right)^{\frac{1}{2}}$ and $x_{1} \in \mathbb{R}^{3}$ be such that $\left|x_{1}\right|=R$. Integrating in time $\left[t_{1}^{\prime \prime}-\frac{T_{1}^{\prime \prime}}{4}, t_{1}^{\prime \prime}\right]$ yields the estimate

$$
\begin{aligned}
M^{-321} e^{C M^{349}} T_{1}^{\frac{1}{2}} e^{-\frac{2 C M^{149} R^{2}}{T_{1}}} & \lesssim M^{-321} T_{1}^{\frac{1}{2}} e^{-\frac{C M^{149} R^{2}}{T_{1}}} \\
& \lesssim \int_{t^{\prime \prime}-T_{1}^{\prime \prime} B_{0}(2 R) \backslash B_{0}(R / 2)}^{t_{1}^{\prime \prime}}|\omega(x, t)|^{2} d x d t
\end{aligned}
$$

which yields the claim (99) of Step 1.

Step 2: quantitative backward uniqueness. The goal of this step and Step 3 below is to prove the following claim:

$$
T_{2}^{-\frac{1}{2}} \exp \left(-\exp \left(M^{1021}\right)\right) \lesssim \int_{B_{0}\left(\frac{3}{4} C(100) M^{1000} R_{2}^{\prime}\right) \backslash B_{0}\left(2 R_{2}^{\prime}\right)}|\omega(x, 0)|^{2} d x,
$$

for all $\frac{8}{C^{\sharp}} M^{749}\left(-t_{0}^{\prime}\right)<T_{2} \leq 1$ and $M$ sufficiently large. Here, $R_{2}, R_{2}^{\prime}$ and $C(100)$ are as in (107)-(109). This is the key estimate for Step 4 below and the proof of Proposition 2.1. 
We apply here the results of Sec. 6 for the quantitative existence of an annulus of regularity. Although the parameter $\mu$ in Sect. 6 is any positive real number, here we need to take $\mu$ sufficiently large in order to have a large enough annulus of quantitative regularity, and hence a large $r_{+}$below in the application of the first Carleman inequality Proposition B.1. To fix the ideas, we take $\mu=100 .{ }^{28}$ Let $T_{1}$ and $T_{2}$ such that

$$
\frac{8}{C^{\sharp}} M^{548+201}\left(-t_{0}^{\prime}\right) \leq T_{2} \leq 1 \text { and } T_{1}:=\frac{T_{2}}{4 M^{201}} \cdot{ }^{29}
$$

Let

$$
R_{2}:=K^{\sharp}\left(T_{2}\right)^{\frac{1}{2}},
$$

for a universal constant $K^{\sharp} \geq 1$ to be chosen sufficiently large below. In particular it is chosen in Step 3 such that (126) holds, which makes it possible to absorb the upper bound (125) of $X_{3}$ in the left hand side of (123). By Corollary 6.7, for $M \geq M_{1}(100)$ there exists a scale

$$
2 R_{2} \leq R_{2}^{\prime} \leq 2 R_{2} \exp \left(C(100) M^{1020}\right)
$$

and a good cylindrical annulus

$$
\mathcal{A}_{2}:=\left\{R_{2}^{\prime}<|x|<c(100) M^{1000} R_{2}^{\prime}\right\} \times\left(-\frac{T_{2}}{32}, 0\right)
$$

such that for $j=0,1$,

$$
\begin{gathered}
\left\|\nabla^{j} u\right\|_{L^{\infty}\left(\mathcal{A}_{2}\right)} \leq 2^{\frac{j+1}{2}} \bar{C}_{j} C(100) M^{-300} T_{2}^{-\frac{j+1}{2}}, \\
\|\nabla \omega\|_{L^{\infty}\left(\mathcal{A}_{2}\right)} \leq 2^{\frac{3}{2}} \bar{C}_{2} C(100) M^{-300} T_{2}^{-\frac{3}{2}} .
\end{gathered}
$$

We apply now the quantitative backward uniqueness, Proposition B.1 to the function $w: \mathbb{R}^{3} \times\left[0, \frac{T_{2}}{M^{201}}\right] \rightarrow \mathbb{R}^{3}$ defined for all $(x, t) \in \mathbb{R}^{3} \times\left[0, \frac{T_{2}}{M^{201}}\right]$ by,

$$
w(x, t)=\omega(x,-t) .
$$

An important remark is that although we have a large cylindrical annulus of quantitative regularity $\mathcal{A}_{2}$, we apply the Carleman estimate on a much smaller annulus, namely

$$
\widetilde{\mathcal{A}}_{2}:=\left\{4 R_{2}^{\prime}<|x|<\frac{c(100)}{4} M^{1000} R_{2}^{\prime}\right\} \times\left(-\frac{T_{2}}{M^{201}}, 0\right) .
$$

Choosing $M$ sufficiently large such that $2 \bar{C}_{j} C(100) M^{-300} \leq 1$ and $2^{\frac{3}{2}} \bar{C}_{2} C(100)$ $M^{-300} \leq 1$, we see that the bounds (110) imply that the differential inequality (307) is satisfied with $S=S_{2}:=\frac{T_{2}}{M^{201}}$ and $C_{C a r l}=M^{201}$. Take

$$
r_{-}=4 R_{2}^{\prime}, \quad r_{+}=\frac{1}{4} c(100) M^{1000} R_{2}^{\prime} .
$$

Then,

$$
B_{0}\left(160 R_{2}^{\prime}\right) \backslash B_{0}\left(40 R_{2}^{\prime}\right)=B_{0}\left(40 r_{-}\right) \backslash B_{0}\left(10 r_{-}\right) \subset\left\{40 R_{2}^{\prime}<|x|<\frac{c(100)}{8} M^{1000} R_{2}^{\prime}\right\}
$$

\footnotetext{
28 More specifically, we see that $\mu$ is chosen so that $10 \mu>350$ in order to obtain (114) from (112) and (113).

29 The reason for this is to ensure we can apply Step 1 to get a lower bound (113) for $Z_{2}$.
} 
on condition that $M$ is sufficiently large: one needs $c(100) M^{1000}>1280$. Note also that

$$
r_{-}^{2}=16\left(R_{2}^{\prime}\right)^{2} \geq 64 R_{2}^{2}=64\left(K^{\sharp}\right)^{2} T_{2}>64 T_{2}>4 S C_{\text {Carl }} .
$$

By (309), we get

$$
Z_{2} \lesssim e^{-\frac{C M^{1000}\left(R_{2}^{\prime}\right)^{2}}{T_{2}}}\left(X_{2}+e^{\frac{C M^{2000}\left(R_{2}^{\prime}\right)^{2}}{T_{2}}} Y_{2}\right)
$$

where

$$
\begin{aligned}
X_{2}:= & \int_{-\frac{T_{2}}{M^{201}}}^{0} \int_{r_{-} \leq|x| \leq r_{+}} e^{\frac{4|x|^{2}}{T_{2}}}\left(M^{201} T_{2}^{-1}|\omega|^{2}+|\nabla \omega|^{2}\right) d x d t, \\
Y_{2}:= & \int_{r_{-} \leq|x| \leq r_{+}}|\omega(x, 0)|^{2} d x, \\
Z_{2}:= & \int_{-\frac{T_{2}}{4 M^{201}}}^{0} \int_{10 r_{-} \leq|x| \leq \frac{r_{+}}{2}}\left(M^{201} T_{2}^{-1}|\omega|^{2}+|\nabla \omega|^{2}\right) d x d t .
\end{aligned}
$$

Thanks to the separation condition (106) and to the fact that for $M$ large enough (107) implies

$$
20 r_{-} \geq 10 R_{2}^{\prime} \geq 20 R_{2}=20 K^{\sharp} T_{2}^{\frac{1}{2}} \geq M^{100}\left(\frac{T_{2}}{8 M^{201}}\right)^{\frac{1}{2}}=M^{100}\left(\frac{T_{1}}{2}\right)^{\frac{1}{2}},
$$

we can apply the concentration result of Step 1 , taking there $T_{1}=\frac{T_{2}}{4 M^{201}}=\frac{S_{2}}{4}$ and $R=20 r_{-}$. By (99) we have that

$$
Z_{2} \gtrsim M^{201}\left(\frac{T_{2}}{4 M^{201}}\right)^{\frac{1}{2}} e^{-\frac{C M^{350}\left(R_{2}^{\prime}\right)^{2}}{T_{2}}} T_{2}^{-1} \gtrsim T_{2}^{-\frac{1}{2}} e^{-\frac{C M^{350}\left(R_{2}^{\prime}\right)^{2}}{T_{2}}} .
$$

Therefore, one of the following two lower bounds holds

$$
\begin{aligned}
& T_{2}^{-\frac{1}{2}} \exp \left(\frac{C M^{1000}\left(R_{2}^{\prime}\right)^{2}}{T_{2}}\right) \lesssim X_{2}, \\
& T_{2}^{-\frac{1}{2}} \exp \left(-\exp \left(M^{1021}\right)\right) \lesssim e^{-\frac{C M^{2000}\left(R_{2}^{\prime}\right)^{2}}{T_{2}}} T_{2}^{-\frac{1}{2}} \lesssim Y_{2},
\end{aligned}
$$

where we used the upper bound (108) for (115). The bound (115) can be used directly in Step 4 below. On the contrary, if (114) holds more work needs to be done to transfer the lower bound to the enstrophy at time 0 . This is the objective of Step 3 below.

Step 3: a final application of quantitative unique continuation. Assume that the bound (114) holds. We will apply the pigeonhole principle three times successively in order to end up in a situation where we can rely on the quantitative unique continuation to get a lower bound at time 0 . We first remark that this, along with the definition (111) of the annulus $\widetilde{\mathcal{A}}_{2}$, implies the following lower bound

$$
T_{2}^{-\frac{1}{2}} \exp \left(\frac{C M^{1000}\left(R_{2}^{\prime}\right)^{2}}{T_{2}}\right)
$$




$$
\lesssim \int_{-\frac{T_{2}}{M^{201}} 4 R_{2}^{\prime} \leq|x| \leq \frac{1}{4} c(100) M^{1000} R_{2}^{\prime}}^{0} \int^{\frac{4|x|^{2}}{T_{2}}}\left(M^{201} T_{2}^{-1}|\omega|^{2}+|\nabla \omega|^{2}\right) d x d t
$$

By the pigeonhole principle, there exists

$$
8 R_{2}^{\prime} \leq R_{3} \leq \frac{1}{2} c(100) M^{1000} R_{2}^{\prime}
$$

such that

$$
T_{2}^{-\frac{1}{2}} \exp \left(-\frac{4 R_{3}^{2}}{T_{2}}\right) \lesssim \int_{-\frac{T_{2}}{M^{201}}}^{0} \int_{B_{0}\left(R_{3}\right) \backslash B_{0}\left(\frac{R_{3}}{2}\right)}\left(T_{2}^{-1}|\omega|^{2}+|\nabla \omega|^{2}\right) d x d t .
$$

Using the bounds (110), we have that

$$
T_{2}^{-\frac{1}{2}} \exp \left(-\frac{4 R_{3}^{2}}{T_{2}}\right) \lesssim \int_{-\frac{T_{2}}{M^{201}}}^{-\exp \left(-\frac{8 R_{3}^{2}}{T_{2}}\right) T_{2}} \int_{B_{0}\left(R_{3}\right) \backslash B_{0}\left(\frac{R_{3}}{2}\right)}\left(T_{2}^{-1}|\omega|^{2}+|\nabla \omega|^{2}\right) d x d t
$$

By the pigeonhole principle, there exists

$$
\frac{1}{2} \exp \left(-\frac{8 R_{3}^{2}}{T_{2}}\right) T_{2} \leq-t_{3} \leq \frac{T_{2}}{M^{201}}
$$

such that

$$
T_{2}^{-\frac{1}{2}} \exp \left(-\frac{5 R_{3}^{2}}{T_{2}}\right) \lesssim \int_{2 t_{3}}^{t_{3}} \int_{B_{0}\left(R_{3}\right) \backslash B_{0}\left(\frac{R_{3}}{2}\right)}\left(T_{2}^{-1}|\omega|^{2}+|\nabla \omega|^{2}\right) d x d t
$$

We finally cover the annulus $B_{0}\left(R_{3}\right) \backslash B_{0}\left(\frac{R_{3}}{2}\right)$ with

$$
C \frac{R_{3}^{3}}{\left(-t_{3}\right)^{\frac{3}{2}}} \lesssim \frac{R_{3}^{3}}{T_{2}^{\frac{3}{2}}} \exp \left(\frac{12 R_{3}^{2}}{T_{2}}\right) \lesssim \exp \left(\frac{13 R_{3}^{2}}{T_{2}}\right)
$$

balls of radius $\left(-t_{3}\right)^{\frac{1}{2}}$, and apply the pigeonhole principle a third time to find that there exists $x_{3} \in B_{0}\left(R_{3}\right) \backslash B_{0}\left(\frac{R_{3}}{2}\right)$ such that

$$
T_{2}^{-\frac{1}{2}} \exp \left(-\frac{18 R_{3}^{2}}{T_{2}}\right) \lesssim \int_{2 t_{3}}^{t_{3}} \int_{B_{x_{3}}\left(\left(-t_{3}\right)^{\frac{1}{2}}\right)}\left(T_{2}^{-1}|\omega|^{2}+|\nabla \omega|^{2}\right) d x d t
$$

We apply now the second Carleman inequality, Proposition B.2, to the function $w$ : $\mathbb{R}^{3} \times\left[0,-20000 t_{3}\right] \rightarrow \mathbb{R}^{3}$ defined for all $(x, t) \in \mathbb{R}^{3} \times\left[0,-20000 t_{3}\right]$ by,

$$
w(x, t)=\omega\left(x+x_{3},-t\right) .
$$


Let $S_{3}:=-20000 t_{3}$. We take to $^{30}$

$$
r_{3}:=1000 R_{3}\left(-\frac{t_{3}}{T_{2}}\right)^{\frac{1}{2}}, \quad \bar{s}_{3}=\underline{s}_{3}=-t_{3} .
$$

Notice that due to (107)-(108) and (116), we have that

$$
\begin{aligned}
& r_{3}^{2}=10^{6} R_{3}^{2}\left(-\frac{t_{3}}{T_{2}}\right) \geq\left(2.56 \times 10^{8}\right)\left(K^{\sharp}\right)^{2}\left(-t_{3}\right) \geq 4000 S_{3}=\left(8 \times 10^{7}\right)\left(-t_{3}\right), \\
& \frac{r_{3}}{2} \geq 8000 R_{2}\left(-\frac{t_{3}}{T_{2}}\right)^{\frac{1}{2}}=8000 K^{\sharp}\left(-t_{3}\right)^{\frac{1}{2}}>\left(-t_{3}\right)^{\frac{1}{2}},
\end{aligned}
$$

so that (311) is satisfied. Furthermore, from (117) we have

$$
\frac{\left|x_{3}\right|}{2} \geq \frac{R_{3}}{4} \geq 1000 R_{3}\left(\frac{1}{M^{201}}\right)^{\frac{1}{2}} \geq r_{3} .
$$

Thus

$$
\begin{aligned}
& B_{x_{3}}\left(\left(-t_{3}\right)^{\frac{1}{2}}\right) \subset B_{x_{3}}\left(\frac{r_{3}}{2}\right) \subset B_{x_{3}}\left(r_{3}\right) \subset B_{x_{3}}\left(\frac{\left|x_{3}\right|}{2}\right) \\
& \subset\left\{\frac{R_{3}}{4}<|y|<\frac{3}{2} R_{3}\right\} \subset\left\{2 R_{2}^{\prime}<|y|<\frac{3}{4} c(100) M^{1000} R_{2}^{\prime}\right\} .
\end{aligned}
$$

Moreover,

$$
0 \leq \bar{s}_{3}=\underline{s}_{3}=-t_{3} \leq-2 t_{3}=\frac{S_{3}}{10^{4}} .
$$

By (117), we see that for $M$ large enough $S_{3} \leq \frac{T_{2}}{32}$, hence the bounds (110) imply that the differential inequality (307) is satisfied on $B_{0}(r) \times[0, S]$ with $S=S_{3}, r=r_{3}$ and $C_{C a r l}=1$. Therefore, by (312) we have

$$
Z_{3} \leq C_{\text {univ }} e^{\frac{r_{3}^{2}}{500 t_{3}}} X_{3}+C_{\text {univ }}\left(-t_{3}\right)^{\frac{3}{2}} e^{-\frac{C r_{3}^{2}}{t_{3}}} Y_{3}
$$

where

$$
\begin{aligned}
& X_{3}:=\int_{-S_{3}}^{0} \int_{B_{x_{3}}\left(r_{3}\right)}\left(S_{3}^{-1}|\omega|^{2}+|\nabla \omega|^{2}\right) d x d t, \quad Y_{3}:=\int_{B_{x_{3}}\left(r_{3}\right)}|\omega(x, 0)|^{2}\left(-t_{3}\right)^{-\frac{3}{2}} e^{\frac{\left|x-x_{3}\right|^{2}}{4 t_{3}}} d x, \\
& Z_{3}:=\int_{2 t_{3}}^{t_{3}} \int_{B_{x_{3}}\left(\frac{r_{3}}{2}\right)}\left(S_{3}^{-1}|\omega|^{2}+|\nabla \omega|^{2}\right) e^{\frac{\left|x-x_{3}\right|^{2}}{4 t}} d x d t .
\end{aligned}
$$

Using (118) and $T_{2}^{-1} \leq S_{3}^{-1}$ we have

$$
T_{2}^{-\frac{1}{2}} \exp \left(-\frac{18 R_{3}^{2}}{T_{2}}\right) \lesssim \int_{2 t_{3}}^{t_{3}} \int_{B_{x_{3}}\left(\left(-t_{3}\right)^{\frac{1}{2}}\right)}\left(T_{2}^{-1}|\omega|^{2}+|\nabla \omega|^{2}\right) e^{\frac{\left|x-x_{3}\right|^{2}}{4 t}} d x d t \leq Z_{3}
$$

30 We follow here an idea of Tao which enables to remove one exponential from the final estimate. This idea appears on his blog https://terrytao.wordpress.com/2019/08/15/quantitative-bounds-for-critically-boundedsolutions-to-the-Navier--Stokes-equations/ in a comment dated December 28, 2019. See also footnote 31 and (127). 
Using the bounds (110) along with (117), we find that

$$
\begin{gathered}
C_{\text {univ }} e^{\frac{r_{3}^{2}}{500 t_{3}}} X_{3} \lesssim S_{3}^{-2} r_{3}^{3} e^{\frac{r_{3}^{2}}{500 t_{3}}} \lesssim\left(-t_{3}\right)^{-\frac{1}{2}} e^{\frac{r_{3}^{2}}{1000 t_{3}}} \lesssim T_{2}^{-\frac{1}{2}} e^{\frac{4 R_{3}^{2}}{T_{2}}} e^{\frac{r_{3}^{2}}{1000 t_{3}}} \\
\lesssim T_{2}^{-\frac{1}{2}} e^{-\frac{996 R_{3}^{2}}{T_{2}}} \lesssim T_{2}^{-\frac{1}{2}} e^{-\frac{18 R_{3}^{2}}{T_{2}}} e^{-\frac{978 R_{3}^{2}}{T_{2}}} \leq C_{\text {univ }}^{\prime} T_{2}^{-\frac{1}{2}} e^{-\frac{18 R_{3}^{2}}{T_{2}}} e^{-978 \cdot 256\left(K^{\sharp}\right)^{2}} .
\end{gathered}
$$

We choose $K^{\sharp}$ sufficiently large so that

$$
C_{\text {univ }}^{\prime} e^{-978 \cdot 256\left(K^{\sharp}\right)^{2}} \leq \frac{1}{2},
$$

where $C_{\text {univ }}^{\prime} \in(0, \infty)$ is the universal constant appearing in the last inequality of (125). Therefore, the term in the right hand side of (125) is negligible with respect to the lower bound (124) of $Z_{3}$. Combining now (123) with the lower bound (124), we obtain ${ }^{31}$

$$
\begin{aligned}
T_{2}^{-\frac{1}{2}} \exp \left(-\frac{18 R_{3}^{2}}{T_{2}}\right) & \lesssim \exp \left(-\frac{C r_{3}^{2}}{t_{3}}\right) \int_{B_{x_{3}}\left(r_{3}\right)}|\omega(x, 0)|^{2} d x \\
& \lesssim \exp \left(C \frac{R_{3}^{2}}{T_{2}}\right) \int_{B_{x_{3}}\left(r_{3}\right)}|\omega(x, 0)|^{2} d x .
\end{aligned}
$$

Hence,

$$
T_{2}^{-\frac{1}{2}} \exp \left(-C \frac{R_{3}^{2}}{T_{2}}\right) \lesssim \int_{B_{x_{3}}\left(r_{3}\right)}|\omega(x, 0)|^{2} d x
$$

Using (107), (122) and the upper bound

$$
R_{3} \leq \frac{1}{2} c(100) M^{1000} R_{2}^{\prime} \leq c(100) M^{1000} \exp \left(C(100) M^{1020}\right) R_{2},
$$

it follows that

$$
T_{2}^{-\frac{1}{2}} \exp \left(-\exp \left(M^{1021}\right)\right) \lesssim \int_{B_{0}\left(\frac{3}{4} c(100) M^{1000} R_{2}^{\prime}\right) \backslash B_{0}\left(2 R_{2}^{\prime}\right)}|\omega(x, 0)|^{2} d x .
$$

Step 4, conclusion: summing the scales and lower bound for the global $L^{3}$ norm. Here we need the extra assumption (49). The key estimate is (105). From (107)-(108), we see that the volume of $B_{0}\left(\frac{3}{4} C(100) M^{1000} R_{2}^{\prime}\right) \backslash B_{0}\left(2 R_{2}^{\prime}\right)$ is less than or equal to $T_{2}^{\frac{3}{2}} \exp \left(M^{1021}\right)$. By the pigeonhole principle, there exists $i \in\{1,2,3\}$ and $x_{4} \in B_{0}\left(\frac{3}{4} C(100) M^{1000} R_{2}^{\prime}\right) \backslash B_{0}\left(2 R_{2}^{\prime}\right)$ such that $\left|\omega_{i}\left(x_{4}, 0\right)\right| \geq 2 T_{2}^{-1} \exp \left(-\exp \left(M^{1022}\right)\right)$.

31 Here one notices a key advantage of taking $r_{3}$ to depend linearly on $\left(-t_{3}\right)^{\frac{1}{2}}$ as in (119). Otherwise the trivial bound

$$
\frac{r_{3}^{2}}{-t_{3}} \leq \frac{R_{3}^{2}}{4\left(-t_{3}\right)} \lesssim \exp \left(\frac{8 R_{3}^{3}}{T_{2}}\right) \frac{R_{3}^{2}}{T_{2}},
$$

where we used the lower bound (117) on $-t_{3}$, would lead one more exponential in the final estimate. Taking $r_{3}$ as in (119) is Tao's idea; see footnote 30 . 
Let $r_{4}:=T_{2}^{\frac{1}{2}} \exp \left(-\exp \left(M^{1022}\right)\right)$. Using (107)-(109), we see that $B_{r_{4}}\left(x_{4}\right) \times\{0\} \subset \mathcal{A}_{2}$. Thus the quantitative estimate (110) gives that

$$
\left|\omega_{i}(x, 0)\right| \geq T_{2}^{-1} \exp \left(-\exp \left(M^{1022}\right)\right) \text { in } B_{r_{4}}\left(x_{4}\right)
$$

and that $\omega_{i}(x, 0)$ has constant sign in $B_{r_{4}}\left(x_{4}\right)$. Without loss of generality, we can take $i=3$. This along with Hölder's inequality yields that

$$
\begin{aligned}
T_{2}^{-1} \exp \left(-\exp \left(M^{1022}\right)\right) & \leq\left|\int_{B_{0}(1)} \omega_{3}\left(x_{4}-r_{4} z, 0\right) \varphi(z) d z\right| \\
& \leq r_{4}^{-1}\left|\int_{B_{0}(1)} u_{2}\left(x_{4}-r_{4} z, 0\right) \partial_{1} \varphi(z)-u_{1}\left(x_{4}-r_{4} z, 0\right) \partial_{2} \varphi(z) d z\right| \\
& \leq r_{4}^{-2}\|u(\cdot, 0)\|_{L^{3}\left(B_{0}\left(C(100) M^{1000} R_{2}^{\prime}\right) \backslash B_{0}\left(R_{2}^{\prime}\right)\right)}\|\nabla \varphi\|_{L^{\frac{3}{2}}\left(B_{0}(1)\right)}
\end{aligned}
$$

for a fixed positive $\varphi \in C_{c}^{\infty}\left(B_{0}(1)\right)$ such that $\int_{B_{0}(1)} \varphi d x=1$. Hence, using (107)-(108) we get

$$
\int \underbrace{}_{B_{0}\left(\exp \left(M^{1023}\right) T_{2}^{\frac{1}{2}}\right) \backslash B_{0}\left(T_{2}^{\frac{1}{2}}\right)}|u(x, 0)|^{3} d x \geq \exp \left(-\exp \left(M^{1023}\right)\right),
$$

for all $\frac{8}{C^{\sharp}} M^{749}\left(-t_{0}^{\prime}\right) \leq T_{2} \leq 1$. Next we divide into two cases.

Case 1: $-t_{0}^{\prime}>\frac{C^{\sharp} \lambda^{2}}{8} M^{-749} \exp \left(-6 M^{1023}\right)$

In this case, we use the additional assumption (49) to immediately get

$$
-t_{0}^{\prime}>\frac{C^{\sharp} \lambda^{2}}{8} M^{-749} \exp \left\{-4 M^{1023} \exp \left(\exp \left(M^{1024}\right)\right) \int_{B_{0}(\lambda)}|u(x, 0)|^{3} d x\right\} .
$$

Case 2: $-t_{0}^{\prime} \leq \frac{C^{\sharp} \lambda^{2}}{8} M^{-749} \exp \left(-6 M^{1023}\right)$

First notice that in this case

$$
M^{-1023} \log \left(\frac{C^{\sharp} \lambda^{2}}{8\left(-t_{0}^{\prime}\right)} M^{-749}\right) \geq 6
$$

which implies

$$
\begin{aligned}
& k+1:=\left\lfloor\frac{1}{2} M^{-1023} \log \left(\frac{C^{\sharp} \lambda^{2}}{8\left(-t_{0}^{\prime}\right)} M^{-749}\right)\right\rfloor \geq 2, \\
& k+1 \geq \frac{1}{4} M^{-1023} \log \left(\frac{C^{\sharp} \lambda^{2}}{8\left(-t_{0}^{\prime}\right)} M^{-749}\right)
\end{aligned}
$$

and

$$
\exp \left((k+1) M^{1023}\right)\left(\frac{8}{C^{\sharp}} M^{749}\left(-t_{0}^{\prime}\right)\right)^{\frac{1}{2}} \leq \lambda<\exp \left(M^{1023}\right) .
$$

In this case we sum (129) on the $k+1 \geq 2$ scales $T_{2}$,

$$
\left(\frac{8}{C^{\sharp}} M^{749}\left(-t_{0}^{\prime}\right)\right)^{\frac{1}{2}} \leq \exp \left(M^{1023}\right)\left(\frac{8}{C^{\sharp}} M^{749}\left(-t_{0}^{\prime}\right)\right)^{\frac{1}{2}}
$$




$$
\leq \ldots \leq \exp \left(k M^{1023}\right)\left(\frac{8}{C^{\sharp}} M^{749}\left(-t_{0}^{\prime}\right)\right)^{\frac{1}{2}} \leq 1 .
$$

Using (131)-(132) we obtain

$$
\begin{aligned}
& \exp \left(-\exp \left(M^{1024}\right)\right) \frac{1}{4} M^{-1023} \log \left(\frac{C^{\sharp} \lambda^{2}}{8} M^{-749}\left(-t_{0}^{\prime}\right)^{-1}\right) \\
& \int|u(x, 0)|^{3} d x . \\
& \int B_{0}(\lambda) \backslash B_{0}\left(\left(\frac{8}{C^{\sharp}} M^{749}\left(-t_{0}^{\prime}\right)\right)^{\frac{1}{2}}\right)
\end{aligned}
$$

This gives

$$
-t_{0}^{\prime} \geq \frac{C^{\sharp} \lambda^{2}}{8} M^{-749} \exp \left\{-4 M^{1023} \exp \left(\exp \left(M^{1024}\right)\right) \int_{B_{0}(\lambda)}|u(x, 0)|^{3} d x\right\},
$$

which was also obtained in Case 1 and hence applies in all cases.

Defining

$$
-s_{\lambda}:=\frac{C^{\sharp} \lambda^{2}}{16} M^{-749} \exp \left\{-4 M^{1023} \exp \left(\exp \left(M^{1024}\right)\right) \int_{B_{0}(\lambda)}|u(x, 0)|^{3} d x\right\},
$$

we see

$$
\left.\int_{B_{0}\left(4 \sqrt{S^{\sharp}}\right.}\left|\omega\left(x, s_{\lambda}\right)\right|^{2} d x \leq M^{2}\right)^{\frac{1}{2}}\left(-s_{\lambda}\right)^{-\frac{1}{2}} \sqrt{S^{\sharp}} .
$$

Almost identical arguments to those utilized in the proof of Lemma 3.1, except using the bound (177) instead of (178), give

$$
\|u\|_{L^{\infty}\left(B_{0}\left(C_{2} M^{50}\left(-s_{\lambda}\right)^{\frac{1}{2}}\right) \times\left(\frac{s_{\lambda}}{4}, 0\right)\right)} \leq \frac{C_{1} M^{-23}}{\left(-s_{\lambda}\right)^{\frac{1}{2}}} .
$$

This concludes the proof of Proposition 2.1.

3.3. Proof of the main estimate in the time slices case. We give the full proof of Proposition 2.2 for the sake of completeness. Notice that the proof follows the same scheme as the proof of Proposition 2.1. The proof of Proposition 2.2 follows from Lemma 3.3, Corollary 6.9, Lemma 7.5 and the Carleman inequalities of "Appendix B". In most estimates $M$ is simply replaced by $M^{b}$, albeit with slightly different powers. However, since in Step 2 below concentration is needed on the very small time interval $\left[-\frac{T_{2}}{4\left(M^{b}\right)^{201}}, 0\right]$, some care is needed when applying Lemma 7.5 on the epoch of quantitative regularity and Lemma 3.3 on the backward propagation of concentration.

Let $M \in\left[M_{4}, \infty\right)$ where $M_{4}$ is a constant in Lemma 3.3. In the course of the proof we will need to take $M$ larger, always larger than universal constants. Let $u$ : $\mathbb{R}^{3} \times[-1,0] \rightarrow \mathbb{R}^{3}$ be a $C^{\infty}\left(\mathbb{R}^{3} \times(-1,0)\right)$ finite-energy solution to the Navier-Stokes equations (1) in $I=[-1,0]$. Assume that there exists $t_{(k)} \in[-1,0)$ such that

$$
t_{(k)} \uparrow 0 \text { with } \sup _{k}\left\|u\left(\cdot, t_{(k)}\right)\right\|_{L^{3}\left(\mathbb{R}^{3}\right)} \leq M .
$$


Select any “well-separated" subsequence (still denoted $\left.t_{(k)}\right)$ such that ${ }^{32}$

$$
\sup _{k} \frac{-t_{(k+1)}}{-t_{(k)}}<\exp \left(-2\left(M^{b}\right)^{1223}\right) .
$$

For this well-separated subsequence, assume that there exists $j+1$ such that the vorticity concentrates at time $t_{(j+1)}$ in the following sense

$$
\int_{B_{0}\left(4 \sqrt{S^{b}}-1\left(-t_{(j+1)}\right)^{\frac{1}{2}}\right)}\left|\omega\left(x, t_{(j+1)}\right)\right|^{2} d x>M^{2}\left(-t_{(j+1)}\right)^{-\frac{1}{2}} \sqrt{S^{b}} .
$$

where we recall that $S^{b}=C M^{-100}$. Fix $k \in\{1,2, \ldots, j\}$. Note that (134) implies that for $M$ sufficiently large

$$
\frac{-t_{(j+1)}}{-t_{(k)}}<\left(M^{b}\right)^{-1051}
$$

Lemma 3.3 then implies that the vorticity concentrates in the following sense

$$
\int_{B_{0}\left(4(-s)^{\frac{1}{2}}\left(M^{b}\right)^{106}\right)}|\omega(x, s)|^{2} d x>\frac{(M+1)^{2}}{(-s)^{\frac{1}{2}}\left(M^{b}\right)^{106}} .
$$

for any

$$
s \in\left[t_{(k)}, \frac{t_{(k)}}{8\left(M^{b}\right)^{201}}\right] .
$$

Step 1: quantitative unique continuation. The purpose of this step is to prove the following estimate:

$$
T_{1}^{\frac{1}{2}} e^{-\frac{C\left(M^{\mathrm{b}}\right)^{965} R^{2}}{T_{1}}} \lesssim \int_{-T_{1} B_{0}(2 R) \backslash B_{0}(R / 2)}^{-\frac{T_{1}}{2}}|\omega(x, t)|^{2} d x d t
$$

for all $T_{1}, s_{0}$ and $R$ such that

$$
s_{0} \in\left[\frac{t_{(k)}}{2}, \frac{t_{(k)}}{4\left(M^{b}\right)^{201}}\right] \quad T_{1}:=-s_{0} \quad \text { and } \quad R \geq\left(M^{b}\right)^{100}\left(\frac{T_{1}}{2}\right)^{\frac{1}{2}} .
$$

Here, $k \in\{1, \ldots j\}$ is fixed. Let $I_{1}:=\left(-T_{1},-\frac{T_{1}}{2}\right) \subset\left[\frac{t_{(k)}}{2}, \frac{t_{(k)}}{8\left(M^{b}\right)^{201}}\right] \subset[-1,0]$. The bound (286) in Remark 7.6 implies that there exists an epoch of regularity $I_{1}^{\prime \prime}=$ $\left[t_{1}^{\prime \prime}-T_{1}^{\prime \prime}, t_{1}^{\prime \prime}\right] \subset I_{1}$ such that

$$
T_{1}^{\prime \prime}=\left|I_{1}^{\prime \prime}\right|=\frac{\left(M^{b}\right)^{-864}}{4 C_{4}^{3}}\left|I_{1}\right|=\frac{\left(M^{b}\right)^{-864}}{8 C_{4}^{3}} T_{1}
$$

\footnotetext{
32 This separation condition is stronger than that of Lemma 3.3. This stronger condition is needed to sum disjoint annuli in Step 4 below.
} 
and for $j=0,1,2$,

$$
\left\|\nabla^{j} u\right\|_{L_{t}^{\infty} L_{x}^{\infty}\left(\mathbb{R}^{3} \times I_{1}^{\prime \prime}\right)} \leq \frac{1}{2^{j+1}}\left|I_{1}^{\prime \prime}\right|^{\frac{-(j+1)}{2}}=\frac{1}{2^{j+1}}\left(T_{1}^{\prime \prime}\right)^{\frac{-(j+1)}{2}} .
$$

Let $T_{1}^{\prime \prime \prime}:=\frac{3}{4} T_{1}^{\prime \prime}$ and $s^{\prime \prime} \in\left[t_{1}^{\prime \prime}-\frac{T_{1}^{\prime \prime}}{4}, t_{1}^{\prime \prime}\right]$. Let $x_{1} \in \mathbb{R}^{3}$ be such that $\left|x_{1}\right| \geq\left(M^{b}\right)^{100}\left(\frac{T_{1}}{2}\right)^{\frac{1}{2}}$ and let $r_{1}:=\left(M^{b}\right)^{50}\left|x_{1}\right| \geq\left(M^{b}\right)^{150}\left(\frac{T_{1}}{2}\right)^{\frac{1}{2}}$. Notice that for $M$ large enough

$$
\frac{\left(M^{b}\right)^{7}\left|x_{1}\right|}{2} \geq \frac{\left(M^{b}\right)^{107}}{2}\left(\frac{T_{1}}{2}\right)^{\frac{1}{2}} \geq 4\left(-s_{0}\right)^{\frac{1}{2}}\left(M^{b}\right)^{106}
$$

and

$$
r_{1}^{2} \geq 4000 T_{1}^{\prime \prime \prime}
$$

We apply the second Carleman inequality, Proposition B.2 (quantitative unique continuation), on the cylinder $\mathcal{C}_{1}=\left\{(x, t) \in \mathbb{R}^{3} \times \mathbb{R}: t \in\left[0, T_{1}^{\prime \prime \prime}\right],|x| \leq r_{1}\right\}$ to the function $w: \mathbb{R}^{3} \times\left[0, T_{1}^{\prime \prime \prime}\right] \rightarrow \mathbb{R}^{3}$, defined for all $(x, t) \in \mathbb{R}^{3} \times\left[0, T_{1}^{\prime \prime \prime}\right]$ by,

$$
w(x, t):=\omega\left(x_{1}+x, s^{\prime \prime}-t\right) .
$$

Notice that the quantitative regularity (141) and the vorticity equation (37) imply that on $\mathcal{C}_{1}$

$$
\left|\left(\partial_{t}+\Delta\right) w\right| \leq \frac{3}{16} T_{1}^{\prime \prime \prime}-1|w|+\frac{\sqrt{3}}{4} T_{1}^{\prime \prime \prime}-\frac{1}{2}|\nabla w|,
$$

so that (310) is satisfied with $S=S_{1}:=T_{1}^{\prime \prime \prime}$ and $C_{\text {Carl }}=\frac{16}{3}$. Let

$$
\bar{s}_{1}=\frac{T_{1}^{\prime \prime \prime}}{20000}, \quad \underline{s}_{1}=\left(M^{b}\right)^{-150} T_{1}^{\prime \prime \prime} .
$$

For $M$ sufficiently large we have $0<\underline{s}_{1} \leq \bar{s}_{1} \leq \frac{T_{1}^{\prime \prime \prime}}{10000}$. Hence by (312) we have

$$
Z_{1} \lesssim e^{-\frac{r_{1}^{2}}{500 \bar{s}_{1}}} X_{1}+\left(\bar{s}_{1}\right)^{\frac{3}{2}}\left(\frac{e \bar{s}_{1}}{\underline{s}_{1}}\right)^{\frac{C r_{1}^{2}}{\bar{s}_{1}}} Y_{1}
$$

where

$$
\begin{aligned}
X_{1}:= & \int_{s^{\prime \prime}-T_{1}^{\prime \prime \prime}} \int_{B_{x_{1}}\left(\left(M^{\mathrm{b}}\right)^{50}\left|x_{1}\right|\right)}\left(\left(T_{1}^{\prime \prime \prime}\right)^{-1}|\omega|^{2}+|\nabla \omega|^{2}\right) d x d s, \\
Y_{1}:= & \int_{B_{x_{1}}\left(\left(M^{\mathrm{b}}\right)^{50}\left|x_{1}\right|\right)}\left|\omega\left(x, s^{\prime \prime}\right)\right|^{2}\left(\underline{s}_{1}\right)^{-\frac{3}{2}} e^{-\frac{\left|x-x_{1}\right|^{2}}{4 \underline{s}_{1}}} d x, \\
Z_{1}:= & \int_{s^{\prime \prime}-\frac{T_{1}^{\prime \prime \prime}}{20000}} \int_{s^{\prime \prime}-\frac{T_{1}^{\prime \prime \prime}}{10000} B_{x_{1}}\left(\frac{\left(M^{\mathrm{b}}\right)^{50}\left|x_{1}\right|}{2}\right)}\left(\left(T_{1}^{\prime \prime \prime}\right)^{-1}|\omega|^{2}+|\nabla \omega|^{2}\right) e^{-\frac{\left|x-x_{1}\right|^{2}}{4\left(s^{\prime \prime}-s\right)}} d x d s .
\end{aligned}
$$


We first use the concentration (136) for times

$$
s \in\left[s^{\prime \prime}-\frac{T_{1}^{\prime \prime \prime}}{10000}, s^{\prime \prime}-\frac{T_{1}^{\prime \prime \prime}}{20000}\right] \subset\left(s_{0}, \frac{s_{0}}{2}\right) \subset\left(t_{(k)}, \frac{t_{(k)}}{8\left(M^{b}\right)^{201}}\right)
$$

to bound $Z_{1}$ from below. By (142), we have

$$
B_{0}\left(4(-s)^{\frac{1}{2}}\left(M^{b}\right)^{106}\right) \subset B_{0}\left(4\left(-s_{0}\right)^{\frac{1}{2}}\left(M^{b}\right)^{106}\right) \subset B_{0}\left(\frac{\left(M^{b}\right)^{7}\left|x_{1}\right|}{2}\right) \subset B_{x_{1}}\left(\left(M^{b}\right)^{7}\left|x_{1}\right|\right)
$$

for all $s \in\left[s^{\prime \prime}-\frac{T_{1}^{\prime \prime \prime}}{10000}, s^{\prime \prime}-\frac{T_{1}^{\prime \prime \prime}}{20000}\right]$ and for $M$ sufficiently large. Hence, we have

$$
\begin{aligned}
Z_{1} & \int_{s^{\prime \prime}-\frac{T_{1}^{\prime \prime \prime}}{10000} B_{0}\left(4(-s)^{\frac{1}{2}}\left(M^{\mathrm{b}}\right)^{106}\right)}^{s^{\prime \prime}-\frac{T_{1}^{\prime \prime \prime}}{20000}}\left(T_{1}^{\prime \prime \prime}\right)^{-1}|\omega(x, s)|^{2} d x d s e^{-\frac{C\left(M^{\mathrm{b}}\right)^{14}\left|x_{1}\right|^{2}}{T_{1}^{\prime \prime \prime}}} \\
& \int_{s^{\prime \prime}-\frac{T_{1}^{\prime \prime \prime}}{20000}} \int_{s^{\prime \prime}-\frac{T_{1}^{\prime \prime \prime}}{10000}}\left(M^{\mathrm{b}}\right)^{-106}(-s)^{-\frac{1}{2}} d s\left(T_{1}^{\prime \prime}\right)^{-1} e^{-\frac{C\left(M^{\mathrm{b}}\right)^{14}\left|x_{1}\right|^{2}}{T_{1}^{\prime \prime}}} \\
& \left(M^{\mathrm{b}}\right)^{-106} \frac{T_{1}^{\prime \prime \prime}}{\left(-s^{\prime \prime}+\frac{T_{1}^{\prime \prime \prime}}{10000}\right)^{\frac{1}{2}}}\left(T_{1}^{\prime \prime}\right)^{-1} e^{-\frac{C\left(M^{\mathrm{b}}\right)^{14}\left|x_{1}\right|^{2}}{T_{1}^{\prime \prime}}} \\
\gtrsim & \left(M^{\mathrm{b}}\right)^{-106}\left(T_{1}\right)^{-\frac{1}{2}} e^{-\frac{C\left(M^{\mathrm{b}}\right)^{14}\left|x_{1}\right|^{2}}{T_{1}^{\prime \prime}}} \\
\gtrsim & \left(M^{\mathrm{b}}\right)^{-106}\left(\left(M^{\mathrm{b}}\right)^{864} T_{1}^{\prime \prime}\right)^{-\frac{1}{2}} e^{-\frac{C\left(M^{\mathrm{b}}\right)^{14}\left|x_{1}\right|^{2}}{T_{1}^{\prime \prime}}} \\
& =\left(M^{\mathrm{b}}\right)^{-538}\left(T_{1}^{\prime \prime}\right)^{-\frac{1}{2}} e^{-\frac{C\left(M^{\mathrm{b}}\right)^{14}\left|x_{1}\right|^{2}}{T_{1}^{\prime \prime}}} .
\end{aligned}
$$

Second, we bound from above $X_{1}$. We rely on the quantitative regularity (141) to obtain

$$
X_{1} \lesssim\left(T_{1}^{\prime \prime}\right)^{-2}\left(M^{b}\right)^{150}\left|x_{1}\right|^{3} .
$$

Hence,

$$
\begin{aligned}
e^{-\frac{r_{1}^{2}}{500 \bar{s}_{1}}} X_{1} & \lesssim\left(T_{1}^{\prime \prime}\right)^{-2}\left(M^{\mathrm{b}}\right)^{150}\left|x_{1}\right|^{3} e^{-\frac{C\left(M^{\mathrm{b}}\right) 100}{T_{1}^{\prime \prime}}} \\
& \lesssim\left(T_{1}^{\prime \prime}\right)^{-\frac{1}{2}} e^{-\frac{C\left(M^{b}\right)^{100}\left|x_{1}\right|^{2}}{T_{1}^{\prime \prime}}} .
\end{aligned}
$$

Third, for $Y_{1}$ we decompose and estimate as follows

$$
\begin{aligned}
Y_{1}:= & \int_{B_{x_{1}}\left(\frac{\left|x_{1}\right|}{2}\right)}\left|\omega\left(x, s^{\prime \prime}\right)\right|^{2}\left(\underline{s}_{1}\right)^{-\frac{3}{2}} e^{-\frac{\left|x-x_{1}\right|^{2}}{4 \underline{s}_{1}}} d x \\
& +\int_{B_{x_{1}}\left(\left(M^{\mathrm{b}}\right)^{50}\left|x_{1}\right|\right) \backslash B_{x_{1}}\left(\frac{\left|x_{1}\right|}{2}\right)}\left|\omega\left(x, s^{\prime \prime}\right)\right|^{2}\left(\underline{s}_{1}\right)^{-\frac{3}{2}} e^{-\frac{\left|x-x_{1}\right|^{2}}{4 \underline{s}_{1}}} d x
\end{aligned}
$$




$$
\begin{aligned}
& \lesssim\left(M^{\mathrm{b}}\right)^{225}\left(T_{1}^{\prime \prime}\right)^{-\frac{3}{2}}\left(\int_{B_{x_{1}}\left(\frac{\left|x_{1}\right|}{2}\right)}\left|\omega\left(x, s^{\prime \prime}\right)\right|^{2} d x\right. \\
& \left.+\int_{B_{x_{1}}\left(\left(M^{\mathrm{b}}\right)^{50}\left|x_{1}\right|\right) \backslash B_{x_{1}}\left(\frac{\left|x_{1}\right|}{2}\right)}\left|\omega\left(x, s^{\prime \prime}\right)\right|^{2} e^{-\frac{C\left(M^{\mathrm{b}}\right)^{150}\left|x_{1}\right|^{2}}{T_{1}^{\prime \prime}}} d x\right) \\
& \lesssim\left(M^{\mathrm{b}}\right)^{225}\left(T_{1}^{\prime \prime}\right)^{-\frac{3}{2}}\left(\int_{B_{x_{1}}\left(\frac{\left|x_{1}\right|}{2}\right)}\left|\omega\left(x, s^{\prime \prime}\right)\right|^{2} d x+\left(M^{\mathrm{b}}\right)^{150}\left|x_{1}\right|^{3}\left(T_{1}^{\prime \prime}\right)^{-2} e^{-\frac{C\left(M^{\mathrm{b}}\right)^{150}\left|x_{1}\right|^{2}}{T_{1}^{\prime \prime}}}\right) \\
& \lesssim\left(M^{\mathrm{b}}\right)^{225}\left(T_{1}^{\prime \prime}\right)^{-\frac{3}{2}}\left(\int_{B_{x_{1}}\left(\frac{\left|x_{1}\right|}{2}\right)}\left|\omega\left(x, s^{\prime \prime}\right)\right|^{2} d x+\left(T_{1}^{\prime \prime}\right)^{-\frac{1}{2}} e^{-\frac{C\left(M^{\mathrm{b}}\right)^{150}\left|x_{1}\right|^{2}}{T_{1}^{\prime \prime}}}\right),
\end{aligned}
$$

where we used the quantitative regularity (141). Hence,

$$
\begin{aligned}
\left(\bar{s}_{1}\right)^{\frac{3}{2}}\left(\frac{e \bar{s}_{1}}{\underline{s}_{1}}\right)^{\frac{C r_{1}^{2}}{\bar{s}_{1}}} Y_{1} \lesssim\left(T_{1}^{\prime \prime}\right)^{\frac{3}{2}} e^{\frac{C\left(M^{\mathrm{b}}\right)^{100}\left|x_{1}\right|^{2}}{T_{1}^{\prime \prime}} \log \left(\frac{e\left(M^{\mathrm{b}}\right) 150}{20000}\right)} Y_{1} \\
\lesssim\left(M^{\mathrm{b}}\right)^{225} e^{\frac{C\left(M^{\mathrm{b}}\right)^{101}\left|x_{1}\right|^{2}}{T_{1}^{\prime \prime}}} \int_{B_{x_{1}}\left(\frac{\left|x_{1}\right|}{2}\right)}\left|\omega\left(x, s^{\prime \prime}\right)\right|^{2} d x \\
+\left(M^{\mathrm{b}}\right)^{225}\left(T_{1}^{\prime \prime}\right)^{-\frac{1}{2}} e^{-\frac{C\left(M^{\mathrm{b}}\right)^{150}\left|x_{1}\right|^{2}}{T_{1}^{\prime \prime}}}
\end{aligned}
$$

Gathering these bounds and combining with (143) yields

$$
\begin{aligned}
\left(M^{\mathrm{b}}\right)^{-538}\left(T_{1}^{\prime \prime}\right)^{-\frac{1}{2}} e^{-\frac{C\left(M^{\mathrm{b}}\right)^{14}\left|x_{1}\right|^{2}}{T_{1}^{\prime \prime}} \lesssim} & \left(T_{1}^{\prime \prime}\right)^{-\frac{1}{2}} e^{-\frac{C\left(M^{\mathrm{b}}\right)^{100}\left|x_{1}\right|^{2}}{T_{1}^{\prime \prime}}} \\
& +\left(M^{\mathrm{b}}\right)^{225} e^{\frac{C\left(M^{\mathrm{b}}\right)^{101}\left|x_{1}\right|^{2}}{T_{1}^{\prime \prime}}} \int_{B_{x_{1}}\left(\frac{\left|x_{1}\right|}{2}\right)}\left|\omega\left(x, s^{\prime \prime}\right)\right|^{2} d x \\
& +\left(M^{\mathrm{b}}\right)^{225}\left(T_{1}^{\prime \prime}\right)^{-\frac{1}{2}} e^{-\frac{\left.C\left(M^{\mathrm{b}}\right)\right)^{150}\left|x_{1}\right|^{2}}{T_{1}^{\prime \prime}}}
\end{aligned}
$$

which implies

$$
\left(M^{b}\right)^{-763}\left(T_{1}^{\prime \prime}\right)^{-\frac{1}{2}} e^{-\frac{C\left(M^{b}\right)^{101}\left|x_{1}\right|^{2}}{T_{1}^{\prime \prime}}} \lesssim \int_{B_{x_{1}}\left(\frac{\left|x_{1}\right|}{2}\right)}\left|\omega\left(x, s^{\prime \prime}\right)\right|^{2} d x .
$$

Hence, for all $s^{\prime \prime} \in\left[t_{1}^{\prime \prime}-\frac{T_{1}^{\prime \prime}}{4}, t_{1}^{\prime \prime}\right]$, for all $\left|x_{1}\right| \geq\left(M^{b}\right)^{100}\left(\frac{T_{1}}{2}\right)^{\frac{1}{2}}$,

$$
\int_{B_{x_{1}}\left(\frac{\left|x_{1}\right|}{2}\right)}\left|\omega\left(x, s^{\prime \prime}\right)\right|^{2} d x \gtrsim\left(M^{b}\right)^{-763}\left(T_{1}^{\prime \prime}\right)^{-\frac{1}{2}} e^{-\frac{C\left(M^{b}\right) 101\left|x_{1}\right|^{2}}{T_{1}^{\prime \prime}}} .
$$


Let $R \geq\left(M^{b}\right)^{100}\left(\frac{T_{1}}{2}\right)^{\frac{1}{2}}$ and $x_{1} \in \mathbb{R}^{3}$ be such that $\left|x_{1}\right|=R$. Integrating in time $\left[t_{1}^{\prime \prime}-\right.$ $\left.\frac{T_{1}^{\prime \prime}}{4}, t_{1}^{\prime \prime}\right]$ yields the estimate

$$
\left(M^{\mathrm{b}}\right)^{-763}\left(T_{1}^{\prime \prime}\right)^{\frac{1}{2}} e^{-\frac{C\left(M^{\mathrm{b}}\right) 101 R^{2}}{T_{1}^{\prime \prime}}} \lesssim \int_{t_{1}^{\prime \prime}-\frac{T_{1}^{\prime \prime}}{4} B_{0}(2 R) \backslash B_{0}(R / 2)}^{t_{1}^{\prime \prime}}|\omega(x, t)|^{2} d x d t,
$$

which yields the claim (138) of Step 1.

Step 2: quantitative backward uniqueness. The goal of this step and Step 3 below is to prove the following claim:

$$
T_{2}^{-\frac{1}{2}} \exp \left(-\exp \left(\left(M^{b}\right)^{1221}\right)\right) \lesssim \int_{B_{0}\left(\frac{3\left(M^{b}\right)^{1200}}{4} R_{2}^{\prime}\right) \backslash B_{0}\left(2 R_{2}^{\prime}\right)}|\omega(x, 0)|^{2} d x,
$$

for $T_{2}=-t_{(k)}$ with $k \in\{1, \ldots j\}$. Here, $R_{2}$ and $R_{2}^{\prime}$ are as in (146)-(147). This is the key estimate for Step 4 below and the proof of Proposition 2.2.

We apply here the results of Sect. 6 for the quantitative existence of an annulus of regularity. Although the parameter $\mu$ in Sect. 6 is any positive real number, here we need to take $\mu$ sufficiently large in order to have a large enough annulus of quantitative regularity, and hence a large $r_{+}$below in the application of the first Carleman inequality, Proposition B.1. To fix the ideas, we take $\mu=120$. Let $T_{1}$ and $T_{2}$ such that

$$
T_{2}:=-t_{(k)} \text { and } T_{1}:=\frac{T_{2}}{4\left(M^{b}\right)^{201}} .
$$

Let

$$
R_{2}:=K^{\mathrm{b}}\left(T_{2}\right)^{\frac{1}{2}}
$$

for a universal constant $K^{b} \geq 1$ to be chosen sufficiently large below. In particular it is chosed such that $(165)$ holds. By Corollary 6.9 applied on the epoch $\left(t_{(k)}, 0\right)$, for $M \geq M_{2}(120)$ there exists a scale

$$
2 R_{2} \leq R_{2}^{\prime} \leq 2 R_{2} \exp \left(C(120)\left(M^{b}\right)^{1220}\right)
$$

and a good cylindrical annulus

$$
\mathcal{A}_{2}:=\left\{R_{2}^{\prime}<|x|<\left(M^{b}\right)^{1200} R_{2}^{\prime}\right\} \times\left(-\frac{T_{2}}{32}, 0\right)
$$

such that for $j=0,1$,

$$
\begin{gathered}
\left\|\nabla^{j} u\right\|_{L^{\infty}\left(\mathcal{A}_{2}\right)} \leq 2^{j+1} \bar{C}_{j}\left(M^{b}\right)^{-360} T_{2}^{-\frac{j+1}{2}}, \\
\|\nabla \omega\|_{L^{\infty}\left(\mathcal{A}_{2}\right)} \leq 2^{\frac{3}{2}} \bar{C}_{2}\left(M^{b}\right)^{-360} T_{2}^{-\frac{3}{2}} .
\end{gathered}
$$

We apply now the quantitative backward uniqueness, Proposition B.1 to the function $w: \mathbb{R}^{3} \times\left[0, \frac{T_{2}}{\left(M^{\mathrm{b}}\right)^{201}}\right] \rightarrow \mathbb{R}^{3}$ defined for all $(x, t) \in \mathbb{R}^{3} \times\left[0, \frac{T_{2}}{\left(M^{\mathrm{b}}\right)^{201}}\right]$ by,

$$
w(x, t)=\omega(x,-t) .
$$


An important remark is that although we have a large cylindrical annulus of quantitative regularity $\mathcal{A}_{2}$, we apply the Carleman estimate on a much smaller annulus, namely

$$
\widetilde{\mathcal{A}}_{2}:=\left\{4 R_{2}^{\prime}<|x|<\frac{\left(M^{b}\right)^{1200}}{4} R_{2}^{\prime}\right\} \times\left(-\frac{T_{2}}{\left(M^{b}\right)^{201}}, 0\right) .
$$

The reason for this is to ensure we can apply Step 1 to get a lower bound (152) for $Z_{2}$.

Choosing $M$ sufficiently large such that $2 \bar{C}_{j}\left(M^{\mathrm{b}}\right)^{-360} \leq 1$ and $2^{\frac{3}{2}} \bar{C}_{2}\left(M^{\mathrm{b}}\right)^{-360} \leq 1$, we see that the bounds (149) imply that the differential inequality (307) is satisfied with $S=S_{2}:=\frac{T_{2}}{\left(M^{b}\right)^{201}}$ and $C_{C a r l}=\left(M^{b}\right)^{201}$. Take

$$
r_{-}=4 R_{2}^{\prime}, \quad r_{+}=\frac{1}{4}\left(M^{b}\right)^{1200} R_{2}^{\prime} .
$$

Then,

$$
B_{0}\left(160 R_{2}^{\prime}\right) \backslash B_{0}\left(40 R_{2}^{\prime}\right)=B_{0}\left(40 r_{-}\right) \backslash B_{0}\left(10 r_{-}\right) \subset\left\{40 R_{2}^{\prime}<|x|<\frac{1}{8}\left(M^{b}\right)^{1200} R_{2}^{\prime}\right\}
$$

provided that $M$ is sufficiently large: one needs $\left(M^{b}\right)^{1200}>1280$. By (309), we get

$$
Z_{2} \lesssim e^{-\frac{C\left(M^{\mathrm{b}}\right)^{1200}\left(R_{2}^{\prime}\right)^{2}}{T_{2}}}\left(X_{2}+e^{\frac{C\left(M^{\mathrm{b}}\right)^{2400}\left(R_{2}^{\prime}\right)^{2}}{T_{2}}} Y_{2}\right)
$$

where

$$
\begin{aligned}
X_{2}:= & \int_{-\frac{T_{2}}{\left(M^{b}\right)^{201}}}^{0} \int_{r_{-} \leq|x| \leq r_{+}} e^{\frac{4|x|^{2}}{T_{2}}}\left(\left(M^{b}\right)^{201} T_{2}^{-1}|\omega|^{2}+|\nabla \omega|^{2}\right) d x d t, \\
Y_{2}:= & \int_{r_{-} \leq|x| \leq r_{+}}|\omega(x, 0)|^{2} d x, \\
Z_{2}:= & \int_{-\frac{T_{2}}{4\left(M^{D}\right)^{201}}}^{0} \int_{0 r_{-} \leq|x| \leq \frac{r_{+}}{2}}\left(\left(M^{b}\right)^{201} T_{2}^{-1}|\omega|^{2}+|\nabla \omega|^{2}\right) d x d t .
\end{aligned}
$$

For $M$ large enough (146) implies

$$
20 r_{-} \geq 10 R_{2}^{\prime} \geq 20 R_{2}=20 K^{b}\left(T_{2}\right)^{\frac{1}{2}} \geq\left(M^{b}\right)^{100}\left(\frac{T_{2}}{8\left(M^{b}\right)^{201}}\right)^{\frac{1}{2}}=\left(M^{b}\right)^{100}\left(\frac{T_{1}}{2}\right)^{\frac{1}{2}} .
$$

Hence, we can apply the concentration result of Step 1 , taking $T_{1}=\frac{T_{2}}{4\left(M^{b}\right)^{201}}=$ $\frac{-t_{(k)}}{4\left(M^{b}\right)^{201}}=\frac{S_{2}}{4}$ and $R=20 r_{-}$. By $(138)$ we have that

$$
Z_{2} \gtrsim\left(M^{\mathrm{b}}\right)^{201}\left(\frac{T_{2}}{4\left(M^{\mathrm{b}}\right)^{201}}\right)^{\frac{1}{2}} e^{-\frac{C\left(M^{\mathrm{b}}\right)^{1166}\left(R_{2}^{\prime}\right)^{2}}{T_{2}}} T_{2}^{-1} \gtrsim T_{2}^{-\frac{1}{2}} e^{-\frac{C\left(M^{\mathrm{b}}\right)^{1166}\left(R_{2}^{\prime}\right)^{2}}{T_{2}}} .
$$

Therefore, one of the following two lower bounds holds

$$
T_{2}^{-\frac{1}{2}} \exp \left(\frac{C M^{1200}\left(R_{2}^{\prime}\right)^{2}}{T_{2}}\right) \lesssim X_{2},
$$




$$
T_{2}^{-\frac{1}{2}} \exp \left(-\exp \left(\left(M^{\mathrm{b}}\right)^{1221}\right)\right) \lesssim e^{-\frac{C\left(M^{\mathrm{b}}\right)^{2400}\left(R_{2}^{\prime}\right)^{2}}{T_{2}}} T_{2}^{-\frac{1}{2}} \lesssim Y_{2},
$$

where we used the upper bound (147) for (154). The bound (154) can be used directly in Step 4 below. On the contrary, if (153) holds more work needs to be done to transfer the lower bound on the enstrophy at time 0 . This is the objective of Step 3 below.

Step 3: a final application of quantitative unique continuation. Assume that the bound (153) holds. We will apply the pigeonhole principle three times successively in order to end up in a situation where we can rely on the quantitative unique continuation to get a lower bound at time 0 . We first remark that this with the definition (150) of the annulus $\widetilde{\mathcal{A}}_{2}$ implies the following lower bound

$$
\begin{aligned}
& T_{2}^{-\frac{1}{2}} \exp \left(\frac{C\left(M^{b}\right)^{1200}\left(R_{2}^{\prime}\right)^{2}}{T_{2}}\right) \\
& \quad \lesssim \int_{-\frac{T_{2}}{\left(M^{b}\right)^{201}} 4 R_{2}^{\prime} \leq|x| \leq \frac{\left(M^{b}\right)^{1200}}{4} R_{2}^{\prime}}^{0} e^{\frac{4|x|^{2}}{T_{2}}}\left(\left(M^{b}\right)^{201} T_{2}^{-1}|\omega|^{2}+|\nabla \omega|^{2}\right) d x d t .
\end{aligned}
$$

By the pigeonhole principle, there exists

$$
8 R_{2}^{\prime} \leq R_{3} \leq \frac{1}{2}\left(M^{b}\right)^{1200} R_{2}^{\prime}
$$

such that

$$
T_{2}^{-\frac{1}{2}} \exp \left(-\frac{4 R_{3}^{2}}{T_{2}}\right) \lesssim \int_{-\frac{T_{2}}{\left(M^{\mathrm{b}}\right)^{201}}}^{0} \int_{B_{0}\left(R_{3}\right) \backslash B_{0}\left(\frac{R_{3}}{2}\right)}\left(T_{2}^{-1}|\omega|^{2}+|\nabla \omega|^{2}\right) d x d t .
$$

Using the bounds (149), we have that

$$
T_{2}^{-\frac{1}{2}} \exp \left(-\frac{4 R_{3}^{2}}{T_{2}}\right) \lesssim \int_{-\frac{T_{2}}{\left(M^{\mathrm{b}}\right)^{201}}}^{-\exp \left(-\frac{8 R_{3}^{2}}{T_{2}}\right) T_{2}} \int_{B_{0}\left(R_{3}\right) \backslash B_{0}\left(\frac{R_{3}}{2}\right)}\left(T_{2}^{-1}|\omega|^{2}+|\nabla \omega|^{2}\right) d x d t
$$

By the pigeonhole principle, there exists

$$
\frac{1}{2} \exp \left(-\frac{8 R_{3}^{2}}{T_{2}}\right) T_{2} \leq-t_{3} \leq \frac{T_{2}}{\left(M^{b}\right)^{201}}
$$

such that

$$
T_{2}^{-\frac{1}{2}} \exp \left(-\frac{5 R_{3}^{2}}{T_{2}}\right) \lesssim \int_{2 t_{3}}^{t_{3}} \int_{B_{0}\left(R_{3}\right) \backslash B_{0}\left(\frac{R_{3}}{2}\right)}\left(T_{2}^{-1}|\omega|^{2}+|\nabla \omega|^{2}\right) d x d t .
$$

We finally cover the annulus $B_{0}\left(R_{3}\right) \backslash B_{0}\left(\frac{R_{3}}{2}\right)$ with

$$
C \frac{R_{3}^{3}}{\left(-t_{3}\right)^{\frac{3}{2}}} \lesssim \frac{R_{3}^{3}}{T_{2}^{\frac{3}{2}}} \exp \left(\frac{12 R_{3}^{2}}{T_{2}}\right) \lesssim \exp \left(\frac{13 R_{3}^{2}}{T_{2}}\right)
$$


balls of radius $\left(-t_{3}\right)^{\frac{1}{2}}$, and apply the pigeonhole principle a third time to find that there exists $x_{3} \in B_{0}\left(R_{3}\right) \backslash B_{0}\left(\frac{R_{3}}{2}\right)$ such that

$$
T_{2}^{-\frac{1}{2}} \exp \left(-\frac{18 R_{3}^{2}}{T_{2}}\right) \lesssim \int_{2 t_{3}}^{t_{3}} \int_{B_{x_{3}}\left(\left(-t_{3}\right)^{\frac{1}{2}}\right)}\left(T_{2}^{-1}|\omega|^{2}+|\nabla \omega|^{2}\right) d x d t
$$

We apply now the second Carleman inequality, Proposition B.2, to the function $w$ : $\mathbb{R}^{3} \times\left[0,-20000 t_{3}\right] \rightarrow \mathbb{R}^{3}$ defined for all $(x, t) \in \mathbb{R}^{3} \times\left[0,-20000 t_{3}\right]$ by,

$$
w(x, t)=\omega\left(x+x_{3},-t\right) .
$$

Let $S_{3}:=-20000 t_{3}$. We take t3 $^{33}$

$$
r_{3}:=1000 R_{3}\left(-\frac{t_{3}}{T_{2}}\right)^{\frac{1}{2}}, \quad \bar{s}_{3}=\underline{s}_{3}=-t_{3} .
$$

Notice that due to (146)-(147) and (155), we have that

$$
\begin{aligned}
& r_{3}^{2}=10^{6} R_{3}^{2}\left(-\frac{t_{3}}{T_{2}}\right) \geq\left(2.56 \times 10^{8}\right)\left(K^{\mathrm{b}}\right)^{2}\left(-t_{3}\right) \geq 4000 S_{3}=\left(8 \times 10^{7}\right)\left(-t_{3}\right), \\
& \frac{r_{3}}{2} \geq 8000 R_{2}\left(-\frac{t_{3}}{T_{2}}\right)^{\frac{1}{2}}=8000 K^{\mathrm{b}}\left(-t_{3}\right)^{\frac{1}{2}}>\left(-t_{3}\right)^{\frac{1}{2}}
\end{aligned}
$$

so that (311) is satisfied. Furthermore, from (156) we have

$$
\frac{\left|x_{3}\right|}{2} \geq \frac{R_{3}}{4} \geq 1000 R_{3}\left(\frac{1}{\left(M^{b}\right)^{201}}\right)^{\frac{1}{2}} \geq r_{3} .
$$

Thus

$$
\begin{aligned}
& B_{x_{3}}\left(\left(-t_{3}\right)^{\frac{1}{2}}\right) \subset B_{x_{3}}\left(\frac{r_{3}}{2}\right) \subset B_{x_{3}}\left(r_{3}\right) \subset B_{x_{3}}\left(\frac{\left|x_{3}\right|}{2}\right) \\
& \subset\left\{\frac{R_{3}}{4}<|y|<\frac{3}{2} R_{3}\right\} \subset\left\{2 R_{2}^{\prime}<|y|<\frac{3\left(M^{b}\right)^{1200}}{4} R_{2}^{\prime}\right\} .
\end{aligned}
$$

Moreover,

$$
0 \leq \bar{s}_{3}=\underline{s}_{3}=-t_{3} \leq-2 t_{3}=\frac{S_{3}}{10^{4}} .
$$

By (156), we see that for $M$ large enough $S_{3} \leq \frac{T_{2}}{32}$, hence the bounds (149) imply that the differential inequality (307) is satisfied with $S=S_{3}$ and $C_{C a r l}=1$. Therefore, by (312) we have

$$
Z_{3} \leq C_{\text {univ }} e^{\frac{r_{3}^{2}}{500 t_{3}}} X_{3}+C_{\text {univ }}\left(-t_{3}\right)^{\frac{3}{2}} e^{-\frac{C r_{3}^{2}}{t_{3}}} Y_{3}
$$

where

$$
X_{3}:=\int_{-S_{3}}^{0} \int_{B_{x_{3}}\left(r_{3}\right)}\left(S_{3}^{-1}|\omega|^{2}+|\nabla \omega|^{2}\right) d x d t, \quad Y_{3}:=\int_{B_{x_{3}}\left(r_{3}\right)}|\omega(x, 0)|^{2}\left(-t_{3}\right)^{-\frac{3}{2}} e^{\frac{\left|x-x_{3}\right|^{2}}{4 t_{3}}} d x,
$$

\footnotetext{
33 As in the proof of Proposition 2.1 above, we follow here again Tao's idea; see footnotes 30 and 31.
} 


$$
Z_{3}:=\int_{2 t_{3}}^{t_{3}} \int_{B_{x_{3}}\left(\frac{r_{3}}{2}\right)}\left(S_{3}^{-1}|\omega|^{2}+|\nabla \omega|^{2}\right) e^{\frac{\left|x-x_{3}\right|^{2}}{4 t}} d x d t
$$

Using (157) and $T_{2}^{-1} \leq S_{3}^{-1}$ we have

$$
T_{2}^{-\frac{1}{2}} \exp \left(-\frac{18 R_{3}^{2}}{T_{2}}\right) \lesssim \int_{2 t_{3}}^{t_{3}} \int_{B_{x_{3}}\left(\left(-t_{3}\right)^{\frac{1}{2}}\right)}\left(T_{2}^{-1}|\omega|^{2}+|\nabla \omega|^{2}\right) e^{\frac{\left|x-x_{3}\right|^{2}}{4 t}} d x d t \leq Z_{3}
$$

Using the bounds (149) along with (156), we find that as in (125),

$$
C_{\text {univ }} e^{\frac{r_{3}^{2}}{500 t_{3}}} X_{3} \lesssim T_{2}^{-\frac{1}{2}} e^{-\frac{996 R_{3}^{2}}{T_{2}}} \leq C_{\text {univ }}^{\prime} e^{-\frac{18 R_{3}^{2}}{T_{2}}} e^{-978 \cdot 256\left(K^{\mathrm{b}}\right)^{2}} .
$$

We choose $K^{b}$ sufficiently large such that

$$
C_{\text {univ }}^{\prime} e^{-978 \cdot 256\left(K^{b}\right)^{2}} \leq \frac{1}{2}
$$

where $C_{\text {univ }}^{\prime} \in(0, \infty)$ is the constant appearing in the last inequality of (164). Combining now (162) with the lower bound (163), we obtain

$$
\begin{aligned}
T_{2}^{-\frac{1}{2}} \exp \left(-\frac{18 R_{3}^{2}}{T_{2}}\right) & \lesssim \exp \left(-\frac{C r_{3}^{2}}{t_{3}}\right) \int_{B_{x_{3}}\left(r_{3}\right)}|\omega(x, 0)|^{2} d x \\
& \lesssim \exp \left(C \frac{R_{3}^{2}}{T_{2}}\right) \int_{B_{x_{3}}\left(r_{3}\right)}|\omega(x, 0)|^{2} d x .
\end{aligned}
$$

Hence,

$$
T_{2}^{-\frac{1}{2}} \exp \left(-C \frac{R_{3}^{2}}{T_{2}}\right) \lesssim \int_{B_{x_{3}}\left(r_{3}\right)}|\omega(x, 0)|^{2} d x
$$

Using (146), (161) and the upper bound

$$
R_{3} \leq \frac{1}{2}\left(M^{b}\right)^{1200} R_{2}^{\prime} \leq\left(M^{b}\right)^{1200} \exp \left(C(120)\left(M^{b}\right)^{1220}\right) R_{2},
$$

it follows that

$$
T_{2}^{-\frac{1}{2}} \exp \left(-\exp \left(\left(M^{\mathrm{b}}\right)^{1221}\right)\right) \lesssim \int_{B_{0}\left(\frac{3\left(M^{\mathrm{b}}\right)^{1200}}{4} R_{2}^{\prime}\right) \backslash B_{0}\left(2 R_{2}^{\prime}\right)}|\omega(x, 0)|^{2} d x .
$$

This together with the bounds (147) and (155) for $R_{3}$ proves the claim (144).

Step 4, conclusion: summing the scales and lower bound for the global $L^{3}$ norm. The key estimate is (144). From (146)-(147), we see that the volume of the annulus 
$B_{0}\left(\frac{3\left(M^{\mathrm{b}}\right)^{1200}}{4} R_{2}^{\prime}\right) \backslash B_{0}\left(2 R_{2}^{\prime}\right)$ is less than or equal to $T_{2}^{\frac{3}{2}} \exp \left(\left(M^{\mathrm{b}}\right)^{1221}\right)$. By the pigeonhole principle, there exist $i \in\{1,2,3\}$ and

$x_{4} \in B_{0}\left(\frac{3\left(M^{b}\right)^{1200}}{4} R_{2}^{\prime}\right) \backslash B_{0}\left(2 R_{2}^{\prime}\right)$ such that $\left|\omega_{i}\left(x_{4}, 0\right)\right| \geq 2 T_{2}^{-1} \exp \left(-\exp \left(\left(M^{b}\right)^{1222}\right)\right)$.

Let $r_{4}:=T_{2}^{\frac{1}{2}} \exp \left(-\exp \left(\left(M^{\mathrm{b}}\right)^{1222}\right)\right)$. Using (146)-(148), we see that $B_{r_{4}}\left(x_{4}\right) \times\{0\} \subset$ $\mathcal{A}_{2}$. Thus the quantitative estimate (149) gives that

$$
\left|\omega_{i}(x, 0)\right| \geq T_{2}^{-1} \exp \left(-\exp \left(\left(M^{b}\right)^{1222}\right)\right) \text { in } B_{r_{4}}\left(x_{4}\right)
$$

and that $\omega_{i}(x, 0)$ has constant sign in $B_{r_{4}}\left(x_{4}\right)$. Without loss of generality, we can take $i=3$. This along with Hölder's inequality yields that

$$
\begin{aligned}
T_{2}^{-1} \exp \left(-\exp \left(\left(M^{\mathrm{b}}\right)^{1222}\right)\right) & \leq\left|\int_{B_{0}(1)} \omega_{3}\left(x_{4}-r_{4} z, 0\right) \varphi(z) d z\right| \\
& \leq r_{4}^{-1}\left|\int_{B_{0}(1)} u_{2}\left(x_{4}-r_{4} z, 0\right) \partial_{1} \varphi(z)-u_{1}\left(x_{4}-r_{4} z, 0\right) \partial_{2} \varphi(z) d z\right| \\
& \leq r_{4}^{-2}\|u(\cdot, 0)\|_{L^{3}\left(B_{0}\left(\left(M^{b}\right)^{1200} R_{2}^{\prime}\right) \backslash B_{0}\left(R_{2}^{\prime}\right)\right)}\|\nabla \varphi\|_{L^{\frac{3}{2}}\left(B_{0}(1)\right)}
\end{aligned}
$$

for a fixed non-negative $\varphi \in C_{c}^{\infty}\left(B_{0}(1)\right)$ such that $\int_{B_{0}(1)} \varphi d x=1$. Recalling (145)(147) we conclude that,

$$
\iint_{B_{0}\left(\exp \left(\left(M^{\mathrm{b}}\right)^{1223}\right)\left(-t_{(k)}\right)^{\frac{1}{2}}\right) \backslash B_{0}\left(\left(-t_{(k)}\right)^{\frac{1}{2}}\right)}|u(x, 0)|^{3} d x \geq \exp \left(-\exp \left(\left(M^{\mathrm{b}}\right)^{1223}\right)\right),
$$

for all $k \in\{1, \ldots j\}$. Note that (134) implies that for distinct $k$, the spatial annuli in (167) are disjoint. Summing (167) over such $k$ we obtain that

$$
\begin{aligned}
& \exp \left(-\exp \left(\left(M^{\mathrm{b}}\right)^{1223}\right)\right) j \\
& \leq \int_{B_{0}\left(\exp \left(\left(M^{\mathrm{b}}\right)^{1223}\right)\left(-t_{1}\right)^{\frac{1}{2}}\right) \backslash B_{0}\left(\left(-t_{(j)}\right)^{\frac{1}{2}}\right)}|u(x, 0)|^{3} d x \\
& \leq \int_{\mathbb{R}^{3}}|u(x, 0)|^{3} d x .
\end{aligned}
$$

This gives

$$
j \leq \exp \left(\exp \left(\left(M^{b}\right)^{1223}\right)\right) \int_{\mathbb{R}^{3}}|u(x, 0)|^{3} d x \leq \exp \left(\exp \left(\left(M^{b}\right)^{1224}\right)\right)
$$

This concludes the proof of Proposition 2.2. 


\section{Further Applications}

4.1. Effective regularity criteria based on the local smallness of the $L^{3, \infty}$ norm at the blow-up time.

Proposition 4.1 For all $M \in[1, \infty)$ sufficiently large the following result holds true. Consider a global-in-time suitable finite-energy solution $(u, p)$ to the Navier-Stokes equations on $\mathbb{R}^{3} \times[-1, \infty)$ that satisfies the following Type I bound

$$
\|u\|_{L_{t}^{\infty} L_{x}^{3, \infty}\left(\mathbb{R}^{3} \times(-1,0)\right)} \leq M .
$$

Assume that for some $T^{*} \in(-1,0]$,

$$
\lim _{r \rightarrow 0}\left\|u\left(\cdot, T^{*}\right)\right\|_{L^{3, \infty}\left(B_{0}(r)\right)} \leq \exp \left(-\exp \left(M^{1023}\right)\right) .
$$

Then, $\left(0, T^{*}\right)$ is a regular point.

Proof of Proposition 4.1. We argue by contradiction and assume $\left(0, T^{*}\right)$ is a singular point. The proof relies on two ingredients: (i) the concentration of the enstrophy norm near a Type I singularity, see Lemma 3.2, (ii) the transfer of concentration at backward times to a lower bound at the final moment in time (Sect. 3.2). Contrary to the proof of Proposition 2.1 no summing of scales argument is required.

Without loss of generality, we assume that $u$ solves Navier-Stokes on $\mathbb{R}^{3} \times(-1,0)$, that $(0,0)$ is a singular point of $u$ and that it satisfies the Type I bound $\|u\|_{L_{t}^{\infty} L_{x}^{3, \infty}\left(\mathbb{R}^{3} \times(-1,0)\right)}$ $\leq M$. First note that by the interpolation inequality for the Lorentz spaces (see Lemma 2.2 in [30] for example) we have that any suitable finite-energy solution with Type I bound is a mild solution on $\mathbb{R}^{3} \times[-1,0]$ with

$$
u \in L_{x, t}^{4}\left(\mathbb{R}^{3} \times(-1,0)\right) .
$$

By Lemma 3.2 and following Step 1-3 in Sect. 3.2, see in particular footnote 27, we can prove that

$$
T_{2}^{-\frac{1}{2}} \exp \left(-\exp \left(M^{1021}\right)\right) \lesssim \int_{B_{0}\left(\exp \left(M^{1021}\right)\left(T_{2}\right)^{\frac{1}{2}}\right) \backslash B_{0}\left(\left(T_{2}\right)^{\frac{1}{2}}\right)}|\omega(x, 0)|^{2} d x,
$$

for all $0<T_{2} \leq 1$ and $M$ sufficiently large. Here we used that $u \in L_{x, t}^{4}\left(\mathbb{R}^{3} \times(-1,0) \cap\right.$ $L_{t}^{\infty} L^{3, \infty}\left(\mathbb{R}^{3} \times(-1,0)\right)$, which allows an application of Corollary 6.7 and Lemma 7.3 in the course of following Steps 1-3.

Let $r \in(0,1]$. Define $T_{2}:=r^{2} \exp \left(-2 M^{1023}\right)$. Following Step 4 of Sect. 3.2 and using Hölder's inequality for Lorentz spaces in Proposition 7.2 instead of Hölder's inequality, we then obtain that

$$
\|u(\cdot, 0)\|_{L^{3, \infty}\left(B_{0}(r)\right)} \geq\|u(\cdot, 0)\|_{L^{3, \infty}\left(B_{0}\left(\exp \left(M^{1023}\right)\left(T_{2}\right)^{\frac{1}{2}}\right) \backslash B_{0}\left(T_{2}^{\frac{1}{2}}\right)\right)} \geq 2 \exp \left(-\exp \left(M^{1023}\right)\right) .
$$

This contradicts (168). 
4.2. Estimate for the number of singular points in a Type I scenario. The technology developed in the present paper also enables us to give an effective bound for the number of singularities in a Type I scenario. The following proposition and its corollary are effective versions of the results by Choe, Wolf and Yang [12] and Seregin [39].

Proposition 4.2 Let $M \in[1, \infty)$ be sufficiently large and define

$$
\varepsilon(M):=\exp \left(-4 \exp \left(M^{1023}\right)\right) .
$$

For all global-in-time suitable finite-energy solutions ${ }^{34}(u, p)$ to the Navier-Stokes equations on $\mathbb{R}^{3} \times[-1, \infty)$ that satisfy the following Type I bound

$$
\|u\|_{L_{t}^{\infty} L_{x}^{3, \infty}\left(\mathbb{R}^{3} \times(-1,0)\right)} \leq M,
$$

the following result holds.

Let $x_{0} \in \mathbb{R}^{3}$. Assume that there exists $r \in\left(0, \exp \left(M^{1021}\right)\right)$ such that

$$
\frac{1}{\left|B_{x_{0}}(r)\right|}\left|\left\{x \in B_{x_{0}}(r):|u(x, 0)| \geq \frac{\varepsilon(M)}{r}\right\}\right| \leq \varepsilon(M) .
$$

Then $\left(x_{0}, 0\right)$ is a regular space-time point.

This result is a variant of Theorem 1 in [12] and Proposition 1.3 in [39]. Our contribution is to provide the explicit formula (171) for $\varepsilon(M)$ in terms of $M$.

Corollary 4.3 Let $T^{*} \in(0, \infty)$ and $M \in[1, \infty)$ be sufficiently large. Assume that $(u, p)$ is a global-in-time suitable finite-energy solution to the Navier-Stokes equations on $\mathbb{R}^{3} \times[0, \infty)$ that satisfies the following Type I bound

$$
\|u\|_{L_{t}^{\infty} L_{x}^{3, \infty}\left(\mathbb{R}^{3} \times\left(0, T^{*}\right)\right)} \leq M .
$$

Then u has at most $\exp \left(\exp \left(M^{1024}\right)\right)$ blow-up points at time $T^{*}$.

Proof of Corollary 4.3. We follow here the argument of [39]. Without loss of generality we can assume that $u$ is defined on $[-1,0]$ rather than $\left[0, T^{*}\right]$. Let $\sigma$ denote the set of all singular points at time 0 . We take a finite collection of $p$ points

$$
x_{1}, \ldots x_{p} \in \sigma
$$

There exists $r \in\left(0, \exp \left(M^{1021}\right)\right)$ such that $B_{x_{i}}(r) \cap B_{x_{j}}(r)=\emptyset$ for all $i \neq j$. Then, Proposition 4.2 implies that

$$
\begin{aligned}
\left|B_{0}(1)\right| \varepsilon(M)^{4} p< & \sum_{i=1}^{p}\left(\frac{\varepsilon(M)}{r}\right)^{3}\left|\left\{x \in B_{x_{i}}(r):|u(x, 0)| \geq \frac{\varepsilon(M)}{r}\right\}\right| \\
& \leq\|u\|_{L_{t}^{\infty} L_{x}^{3, \infty}\left(\mathbb{R}^{3} \times(-1,0)\right)}^{3} \leq M^{3}
\end{aligned}
$$

This yields the result.

\footnotetext{
34 For a definition of 'suitable finite-energy solutions' we refer to Sect. 1.4 'Notations'.
} 
Proof of Proposition 4.2. Without loss of generality we assume that $x_{0}=0$. As in the proof of Proposition 4.1, we assume for contradiction that $(0,0)$ is a singular point. Using verbatim reasoning as in the proof of Proposition 4.1, we see that the outcome of Step 1-3 in Sect. 3.2 holds, in particular estimate (105), which holds for all $0<T_{2} \leq 1$.

Arguing as in Step 4, and using the same notation, we get that there exists

$$
x_{4} \in B_{0}\left(\frac{3}{4} C(100) M^{1000} R_{2}^{\prime}\right) \backslash B_{0}\left(2 R_{2}^{\prime}\right)
$$

such that for $r_{4}:=T_{2}^{\frac{1}{2}} \exp \left(-\exp \left(M^{1022}\right)\right)$,

$$
\begin{aligned}
\exp \left(-\exp \left(M^{1023}\right)\right) & \leq \int_{B_{x_{4}}\left(r_{4}\right)}|u(x, 0)|^{3} d x \\
& \leq T_{2}^{\frac{3}{2}} \exp \left(-3 \exp \left(M^{1022}\right)\right) \sup _{B_{x_{4}}\left(r_{4}\right)}|u(x, 0)|^{3} .
\end{aligned}
$$

Hence, there exists $x_{5} \in B_{x_{4}}\left(r_{4}\right)$ such that

$$
\left|u\left(x_{5}, 0\right)\right| \geq 2 T_{2}^{-\frac{1}{2}} \exp \left(-\frac{1}{3} \exp \left(M^{1023}\right)\right) .
$$

By estimate (110) and the choice of $M$ sufficiently large, we have $\|\nabla u\|_{L^{\infty}\left(\mathcal{A}_{2}\right)} \leq 1$ in the good annulus. Hence, for $r_{5}:=T_{2}^{\frac{1}{2}} \exp \left(-\exp \left(M^{1023}\right)\right)$, the ball $B_{x_{5}}\left(r_{5}\right)$ is contained in $\mathcal{A}_{2}$ and

$$
|u(x, 0)| \geq T_{2}^{-\frac{1}{2}} \exp \left(-\exp \left(M^{1023}\right)\right) \quad \text { in } \quad B_{x_{5}}\left(r_{5}\right)
$$

for all $0<T_{2} \leq 1$. For $r:=T_{2}^{\frac{1}{2}} \exp \left(M^{1021}\right)$, we have $B_{x_{5}}\left(r_{5}\right) \subset \mathcal{A}_{2} \subset B_{0}(r)$ and

$$
|u(x, 0)| \geq \frac{\exp \left(-\exp \left(M^{1023}\right)\right)}{r} .
$$

Subsequently,

$$
\begin{aligned}
& \frac{1}{\left|B_{0}(r)\right|}\left|\left\{x \in B_{0}(r):|u(x, 0)| \geq \frac{\exp \left(-\exp \left(M^{1023}\right)\right)}{r}\right\}\right| \\
& \geq\left(\frac{r_{5}}{r}\right)^{3}>\exp \left(-4 \exp \left(M^{1023}\right)\right)
\end{aligned}
$$

This holds for $r=T_{2}^{\frac{1}{2}} \exp \left(M^{1021}\right)$ and every $0<T_{2} \leq 1$, which contradicts our assumption (172) on $u(\cdot, 0)$. 
4.3. Effective regularity criteria based on the relative smallness of the $L^{3}$ norm at the final moment in time. Here we prove an effective regularity criteria for $(u, p)$ a solution to the Navier-Stokes equations on $\mathbb{R}^{3} \times[-1, \infty)$ based on the relative smallness of $\|u(\cdot, 0)\|_{L^{3}}$ vs. $\|u(\cdot,-1)\|_{L^{3}}$. A non-effective version of this result (without explicit quantitative bounds) is in [2, Theorem 4.1 (i)].

Proposition 4.4 For all sufficiently large $M \in[1, \infty)$, we define $M^{b}$ by (17). Let $(u, p)$ be a global-in-time suitable finite-energy solution to the Navier-Stokes equations (1) on $\mathbb{R}^{3} \times[-1, \infty)$. Assume that

$$
\|u(\cdot,-1)\|_{L^{3}\left(\mathbb{R}^{3}\right)} \leq M .
$$

If

$$
\|u(\cdot, 0)\|_{L^{3}\left(B_{0}\left(\exp \left(\left(M^{\mathrm{b}}\right)^{1221}\right)\right) \backslash B_{0}(1)\right)} \leq \exp \left(-\exp \left(\left(M^{\mathrm{b}}\right)^{1223}\right)\right),
$$

then $(0,0)$ is a regular point.

Proof. Assume for contradiction that $(0,0)$ is a singular point. Since $(u, p)$ is a suitable finite-energy solution, there exists $\Sigma \subset(-1,0)$ such that $|\Sigma|=1$ and

- $\left\|\nabla u\left(\cdot, t^{\prime}\right)\right\|_{L^{2}\left(\mathbb{R}^{3}\right)}<\infty$ for all $t^{\prime} \in \Sigma$,

- $u$ satisfies the energy inequality on $\left[t^{\prime}, 0\right]$.

Then, arguing in a similar way as in the proof of Lemma 3.3, we show that for any $s_{0} \in\left[-1,-\frac{1}{8\left(M^{b}\right)^{201}}\right] \cap \Sigma$ the vorticity concentrates in the following sense,

$$
\int_{B_{0}\left(4\left(-s_{0}\right)^{\frac{1}{2}}\left(M^{b}\right)^{106}\right)}\left|\omega\left(x, s_{0}\right)\right|^{2} d x>\frac{(M+1)^{2}}{\left(-s_{0}\right)^{\frac{1}{2}}\left(M^{b}\right)^{106}} .
$$

Here, $M^{b}$ is as in (17).

Using $|\Sigma|=1$ and then following Step 1-3 of Sect. 3.3 with one time scale, we obtain

$$
T_{2}^{-\frac{1}{2}} \exp \left(-\exp \left(\left(M^{\mathrm{b}}\right)^{1221}\right)\right) \lesssim \int_{B_{0}\left(\frac{3\left(M^{\mathrm{b}}\right)^{1200}}{4} R_{2}^{\prime}\right) \backslash B_{0}\left(2 R_{2}^{\prime}\right)}|\omega(x, 0)|^{2} d x
$$

for

$$
T_{2}=1, \quad R_{2}:=K^{\mathrm{b}}\left(T_{2}\right)^{\frac{1}{2}}, \quad 2 R_{2} \leq R_{2}^{\prime} \leq 2 R_{2} \exp \left(C(120)\left(M^{\mathrm{b}}\right)^{1220}\right)
$$

for $K^{b}$ chosen such that (165) holds. Reasoning as in Step 4 of Sect. 3.3, we then obtain

$$
\int_{B_{0}\left(\exp \left(\left(M^{\mathrm{b}}\right)^{1221}\right)\right) \backslash B_{0}(1)}|u(x, 0)|^{3} d x \geq 2 \exp \left(-\exp \left(\left(M^{\mathrm{b}}\right)^{1223}\right)\right) .
$$

This concludes the proof. 


\section{Main Tool 1: Local-in-Space Short-Time Smoothing}

The role of the next result is central in our paper.

Theorem 5.1 (local-in-space short-time smoothing). There exist three universal constants $C_{*}, M_{5}, N_{1} \in[1, \infty)$ such that the following holds. For all $M \geq M_{5}, N \geq$ $N_{1}$, there exists a time $S_{*}(M, N) \in\left(0, \frac{1}{4}\right]$ such that the following holds. Consider a divergence-free initial data $u_{0}$ satisfying the global control

$$
\left\|u_{0}\right\|_{L_{\text {uloc }}^{2}\left(\mathbb{R}^{3}\right)} \leq M, \quad\left\|u_{0}\right\|_{L^{2}\left(B_{\bar{x}}(1)\right)} \stackrel{|\bar{x}| \rightarrow \infty}{\longrightarrow} 0,
$$

and, in addition, $u_{0} \in L^{6}\left(B_{0}(2)\right)$ with

$$
\left\|u_{0}\right\|_{L^{6}\left(B_{0}(2)\right)} \leq N .
$$

Then, for any local energy solution ${ }^{35}(u, p)$ to (1) with initial data $u_{0}$ we have the estimate

$$
\begin{aligned}
& \|u\|_{L^{\infty}\left(B_{0}\left(\frac{1}{2}\right) \times\left(\frac{3}{4} S_{*}, S_{*}\right)\right)} \leq C_{*} M^{8} N^{19}, \\
& \|\nabla u\|_{L_{t}^{\infty} L_{x}^{2}\left(B_{0}\left(\frac{1}{4}\right) \times\left(\frac{15}{16} S_{*}, S_{*}\right)\right)} \leq C_{*} M^{40} N^{98} .
\end{aligned}
$$

Moreover, there is an explicitformulafor $S_{*}$, see (209), and $S_{*}(M, N)=C M^{-30} N^{-70}$. Remark 5.2. As a conclusion to the hypothesis in the above Theorem, one can also obtain general version of (178). Specifically, for a local energy solution with $\beta \in\left(0, S_{*}\right]$, we get

$$
\begin{aligned}
& \|\nabla u\|_{L_{t}^{\infty} L_{x}^{2}\left(B_{0}\left(\frac{1}{6}\right) \times\left(\frac{255}{256} \beta, \beta\right)\right)} \\
& \quad \leq C_{*} \beta^{-\frac{3}{4}}\left(M N^{2}+M N \beta^{-\frac{1}{4}}+M \beta^{-\frac{1}{2}}+M^{2}+\left(N^{2}+N \beta^{-\frac{1}{4}}\right)^{2}\right) .
\end{aligned}
$$

We will require this more general estimate. The computations producing it are identical to those used to show Theorem 5.1 and hence are omitted.

Corollary 5.3 There exist two universal constants $C_{* *}, M_{6} \in[1, \infty)$ such that the following holds. For all $M \geq M_{6}$ there exists a time $S_{* *}(M) \in\left(0, \frac{1}{4}\right]$ with $S_{* *}(M)=$ $C M^{-100}$ (given explicitly by (183)) such that the following holds. Suppose $(u, p)$ is a 'smooth solution with sufficient decay ${ }^{36}$ on $\mathbb{R}^{3} \times\left[0, T^{\prime}\right]$ for any $T^{\prime} \in(0, T)$ and satisfies

$$
\|u\|_{L_{t}^{\infty} L_{x}^{3, \infty}\left(\mathbb{R}^{3} \times[0, T]\right)} \leq M .
$$

Furthermore, suppose there exists $t \in(0, T)$ such that

$$
\|u(\cdot, t)\|_{L^{\infty}\left(B_{0}\left(2 \sqrt{S_{* *}}-1(T-t)^{\frac{1}{2}}\right)\right)} \leq \frac{M \sqrt{S_{* *}}}{(T-t)^{\frac{1}{2}}} .
$$

Then we conclude that

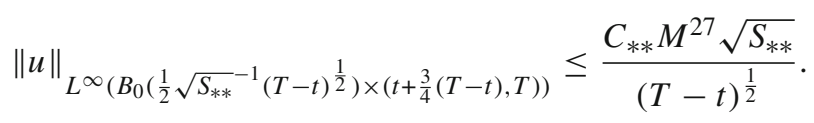

35 We recall that the definition of a 'local energy solution' is given in Sect. 1.4.

${ }^{36}$ See Sect. 1.4. 
Proof. We define $S_{* *} \in\left(0, \frac{1}{4}\right]$ in the following way:

$$
S_{* *}=S_{* *}(M):=S_{*}\left(C_{\text {weak }} M,\left|B_{0}(2)\right|^{\frac{1}{6}} M\right),
$$

where $S_{*}$ is the function defined in Theorem 5.1 (see also the formula (209)), and $C_{\text {weak }}$ is defined below (186). Define

$$
r:={\sqrt{S_{* *}}}^{-1}(T-t)^{\frac{1}{2}}
$$

and rescale

$$
U(y, s):=r u\left(r y, r^{2} s+t\right) \text { for }(y, s) \in \mathbb{R}^{3} \times\left(0, S_{* *}\right) .
$$

Then assumptions (180)-(181) imply that

$$
\|U(\cdot, 0)\|_{L^{\infty}\left(B_{0}(2)\right)} \leq M \text { and }\|U\|_{L_{t}^{\infty} L_{x}^{3, \infty}\left(\mathbb{R}^{3} \times\left(0, S_{* *}\right)\right)} \leq M .
$$

Hence we have,

and

$$
\|U(\cdot, 0)\|_{L^{6}\left(B_{0}(2)\right)} \leq M\left|B_{0}(2)\right|^{\frac{1}{6}}
$$

$$
\|U(\cdot, 0)\|_{L_{\text {uloc }}^{2}\left(\mathbb{R}^{3}\right)} \leq C_{\text {weak }} M .
$$

Here, $C_{\text {weak }} \in[1, \infty)$ is a universal constant from the embedding $L^{3, \infty}\left(\mathbb{R}^{3}\right) \subset L_{\text {uloc }}^{2}\left(\mathbb{R}^{3}\right)$. We then apply Theorem 5.1 to $U$ and then rescale according to (184). This gives (182) as desired.

Theorem 5.1 is proven in Sect. 5.2 below. It relies on an $\varepsilon$-regularity result for suitable weak solutions ${ }^{37}$ to the perturbed Navier-Stokes equations

$$
\partial_{t} v-\Delta v+\nabla q=-v \cdot \nabla v-a \cdot \nabla v-\nabla \cdot(a \otimes v), \quad \nabla \cdot v=0, \quad \nabla \cdot a=0
$$

around a subcritical drift $a \in L^{m}\left(Q_{(0,0)}(1)\right), m>5$. We recover the result of Jia and Šverák [20, Theorem 2.2] by a Caffarelli, Kohn and Nirenberg scheme [9] already used in [6] for critical drifts; see also [21] for a similar result obtained by a compactness argument. Contrary to the critical case, here we can prove boundedness directly.

Theorem 5.4 (epsilon-regularity around a subcritical drift). There exists $C_{* * *} \in(0, \infty)$, for all $m \in(5, \infty]$, there exists $\varepsilon_{*}(m) \in(0, \infty)$ such that the following holds for all $\varepsilon \in\left(0, \varepsilon_{*}(m)\right)$. Take any $a \in L^{m}\left(Q_{(0,0)}(1)\right)$ and any suitable weak solution $(v, q)$ to (187) satisfying

$$
\sup _{-1<s<0} \int_{B_{0}(1)}|v(x, s)|^{2} d x+\int_{Q_{(0,0)}(1)}|\nabla v|^{2} d x d s \leq \varepsilon^{\frac{5}{9}} .
$$

Assume that

$$
\begin{gathered}
\|a\|_{L^{m}\left(Q_{(0,0)}(1)\right)} \leq \varepsilon^{\frac{1}{9}} \\
\int_{Q_{(0,0)}(1)}|v|^{3}+|q|^{\frac{3}{2}} d x d s \leq \varepsilon
\end{gathered}
$$

Then,

$$
\sup _{(\bar{x}, t) \in \overline{Q_{(0,0)}\left(\frac{1}{2}\right)}} \sup _{r \in\left(0, \frac{1}{4}\right]} f_{Q_{(\bar{x}, t)}(r)}|v|^{3} d x d s \leq C_{* * *} \varepsilon^{\frac{2}{3}} .
$$

\footnotetext{
37 For a definition of suitable weak solutions for (187), we refer to [6, Definition 1].
} 
This theorem is proved in Sect. 5.1 below. Notice that the smallness on the largescale quantity (190) in $Q_{(0,0)}(1)$ is transferred to the $L^{\infty}$ bound (191). In the following statement, we remove the smallness assumption (189) on the drift.

Corollary 5.5 Let $m \in(5, \infty]$. Let $C_{* * *}$ and $\varepsilon_{*}$ be given by Theorem 5.4. For all $\varepsilon \in\left(0, \min \left(\varepsilon_{*}, 2^{-9}\right)\right)$, for all $N \in[1, \infty)$, for all $a \in L^{m}\left(Q_{(0,0)}(1)\right)$ and any suitable weak solution $(v, q)$ to $(187)$ satisfying

$$
\sup _{-1<s<0} \int_{B_{0}(1)}|v(x, s)|^{2} d x+\int_{Q_{(0,0)}(1)}|\nabla v|^{2} d x d s \leq N^{-\frac{1}{1-\frac{5}{m}}} \varepsilon^{\frac{1}{9} \cdot \frac{6-\frac{25}{m}}{1-\frac{5}{m}}}
$$

the following holds. Assume that

$$
\begin{aligned}
& \|a\|_{L^{m}\left(Q_{(0,0)}(1)\right)} \leq N, \\
& \int_{Q_{(0,0)}(1)}|v|^{3}+|q|^{\frac{3}{2}} d x d s \leq N^{-\frac{2}{1-\frac{5}{m}}} \varepsilon^{\frac{1}{9} \cdot \frac{11-\frac{45}{m}}{1-\frac{5}{m}}} .
\end{aligned}
$$

Then,

$$
\|v\|_{L^{\infty}\left(Q_{(0,0)}\left(\frac{1}{2}\right)\right)} \leq C_{* * *}^{\frac{1}{3}} N^{\frac{1}{1-\frac{5}{m}}} \varepsilon^{\frac{1}{9} \cdot \frac{1-\frac{10}{m}}{1-\frac{5}{m}}} .
$$

Proof of Corollary 5.5. We use a scaling argument as in [20, Theorem 2.2]. Let $\left(x_{0}, t_{0}\right) \in$ $Q_{(0,0)}\left(\frac{1}{2}\right)$ and define $R_{0} \in(0, \infty)$ as

$$
R_{0}=N^{-\frac{1}{1-\frac{5}{m}}} \varepsilon^{\frac{1}{9} \cdot \frac{1}{1-\frac{5}{m}}}
$$

Notice that due to $0<\varepsilon<\min \left(\varepsilon_{*}, 2^{-9}\right)$, we have $R_{0}<\frac{1}{2}$, so that the following rescaling is well defined: for all $(y, s) \in Q_{(0,0)}(1)$,

$$
V(y, s):=R_{0} v\left(x_{0}+R_{0} y, t_{0}+R_{0}^{2} s\right), \quad Q(y, s):=R_{0}^{2} q\left(x_{0}+R_{0} y, t_{0}+R_{0}^{2} s\right) .
$$

Then $(V, Q)$ is a suitable weak solution to (187) with a drift $b$ defined by

$$
b(y, s):=R_{0} a\left(x_{0}+R_{0} y, t_{0}+R_{0}^{2} s\right) .
$$

We have by our choice of $R_{0}$ in (196)

$$
\|b\|_{L^{m}\left(Q_{(0,0)}(1)\right)} \leq R_{0}^{1-\frac{5}{m}}\|a\|_{L^{m}\left(Q_{\left(x_{0}, t_{0}\right)}\left(R_{0}\right)\right)} \leq R_{0}^{1-\frac{5}{m}}\|a\|_{L^{m}\left(Q_{(0,0)}(1)\right)} \leq R_{0}^{1-\frac{5}{m}} N \leq \varepsilon^{\frac{1}{9}}
$$

for the drift,

$$
\begin{aligned}
& \sup _{-1<s<0} \int_{B_{0}(1)}|V(y, s)|^{2} d y+\int_{Q_{(0,0)}(1)}|\nabla V|^{2} d y d s \\
& \leq R_{0}^{-1}\left(\sup _{t_{0}-R_{0}^{2}<s<t_{0}} \int_{B_{x_{0}}\left(R_{0}\right)}|v(x, s)|^{2} d x+\int_{Q_{\left(x_{0}, t_{0}\right)}\left(R_{0}\right)}|\nabla v|^{2} d x d s\right)
\end{aligned}
$$




$$
\begin{aligned}
& \leq R_{0}^{-1}\left(\sup _{-1<s<0} \int_{B_{0}(1)}|v(x, s)|^{2} d x+\int_{Q_{(0,0)}(1)}|\nabla v|^{2} d x d s\right) \\
& \leq R_{0}^{-1} N^{-\frac{1}{1-\frac{5}{m}}} \varepsilon^{\frac{1}{9} \cdot \frac{6-\frac{25}{m}}{1-\frac{5}{m}}}=\varepsilon^{\frac{5}{9}}
\end{aligned}
$$

for the local energy and finally

$$
\begin{aligned}
& \int_{Q_{(0,0)}(1)}|V|^{3}+|Q|^{\frac{3}{2}} d y d s \\
& \leq R_{0}^{-2} \int_{Q_{\left(x_{0}, t_{0}\right)}\left(R_{0}\right)}|v|^{3}+|q|^{\frac{3}{2}} d x d s \\
& \leq R_{0}^{-2} \int_{Q_{(0,0)}(1)}|v|^{3}+|q|^{\frac{3}{2}} d x d s \\
& \leq R_{0}^{-2} N^{-\frac{2}{1-\frac{5}{m}}} \varepsilon^{\frac{1}{9} \cdot \frac{11-\frac{45}{m}}{1-\frac{5}{m}}}=\varepsilon .
\end{aligned}
$$

Therefore, (188), (189) and (190) are satisfied for ( $V, Q)$, and hence,

$$
\sup _{(\bar{x}, t) \in \sup _{(0,0)}\left(\frac{1}{2}\right)} \int_{r \in\left(0, \frac{1}{4}\right]} f_{Q_{(\bar{x}, t)}(r)}|V|^{3} d y d s \leq C_{* * *} \varepsilon^{\frac{2}{3}} .
$$

Rescaling, this gives

$$
\sup _{(\bar{x}, t) \in Q_{\left(x_{0}, t_{0}\right)}\left(\frac{R_{0}}{2}\right)} \sup _{r \in\left(0, \frac{R_{0}}{4}\right]} f_{Q_{(\bar{x}, t)}(r)}|v|^{3} d x d s \leq C_{* * *} \varepsilon^{\frac{2}{3}} R_{0}^{-3},
$$

hence we obtain the bound (195) by taking the supremum over $\left(x_{0}, t_{0}\right) \in Q_{(0,0)}\left(\frac{1}{2}\right)$. This concludes the proof.

5.1. Sketch of the proof of Theorem 5.4. The proof follows almost verbatim the one of Theorem 3 in [6], provided the following modifications are made. We propagate the following two bounds: for $r_{k}=2^{-k}$,

$$
\begin{gathered}
\frac{1}{r_{k}^{2}} \int_{Q_{(\bar{x}, t)}\left(r_{k}\right)}|v(x, s)|^{3} d x d s+\frac{1}{r_{k}^{\frac{1}{2}+\kappa}} \int_{Q_{(\bar{x}, t)}\left(r_{k}\right)}\left|q-(q)_{r_{k}}(s)\right|^{\frac{3}{2}} d x d s \leq \varepsilon^{\frac{2}{3}} r_{k}^{3}, \\
\sup _{t-r_{k}<s<t} \int_{B_{\bar{x}}\left(r_{k}\right)}|v(x, s)|^{2} d x+\int_{Q_{(\bar{x}, t)}\left(r_{k}\right)}|\nabla v|^{2} d x d s \leq C_{B} \varepsilon^{\frac{2}{3}} r_{k}^{3},
\end{gathered}
$$

for a universal constant $C_{B} \in(0, \infty)$ chosen sufficiently large and $\kappa(m) \in(0, \infty)$ such that

$$
0<\kappa<\min \left(2,3-\frac{15}{m}\right) \text {. }
$$


One takes advantage of the subcriticality of the drift $a$ in the following way:

$$
\|a\|_{L^{5}\left(Q_{(\bar{x}, t)}\left(r_{k}\right)\right)} \lesssim r_{k}^{1-\frac{5}{m}}\|a\|_{L^{m}\left(Q_{(\bar{x}, t)}\left(r_{k}\right)\right)} \lesssim \varepsilon^{\frac{1}{9}} r_{k}^{1-\frac{5}{m}}
$$

This plays a key role in the estimate of $I_{4}$ and $I_{5}$ in Step 3, $J_{2}$ and $J_{4}$ in Step 4, using the same notations as in the proof of [6, Theorem 3]. The restriction $\kappa<2$ comes from handling $J_{5}$ and $J_{6}$, while the restriction $\kappa<3-\frac{15}{m}$ comes from bounding $J_{2}$ and $J_{4}$.

5.2. Proof of Theorem 5.1. We fix $n=6$ and $m=\frac{5 n}{3}=10$ in this proof. Let $C_{* * *}$ and $\varepsilon_{*}=\varepsilon_{*}(10)$ be given by Theorem 5.4. We fix $\varepsilon=\varepsilon_{*}(10) / 2$ for the whole proof. Let also $k_{0}=k_{0}(6)$ and $K_{0}=K_{0}(6)$ be given by Proposition A.1.

Let $M, N \in[1, \infty)$. Let $u_{0} \in L_{u l o c}^{2}\left(\mathbb{R}^{3}\right)$ be such that $\left\|u_{0}\right\|_{L^{2}\left(B_{\bar{x}}(1)\right)} \stackrel{|\bar{x}| \rightarrow \infty}{\longrightarrow} 0$. We assume in addition that $u_{0} \in L^{6}\left(B_{0}(2)\right)$. Moreover,

$$
\left\|u_{0}\right\|_{L_{u l o c}^{2}\left(\mathbb{R}^{3}\right)} \leq M, \quad\left\|u_{0}\right\|_{L^{6}\left(B_{0}(2)\right)} \leq N .
$$

Let $u$ be any local energy solution to (1) with such a data $u_{0}$. The goal is to prove the local-in-space short-time smoothing for $u$ stated in Theorem 5.1.

\section{Step 1: decomposition of the initial data.}

Lemma 5.6 Let $u_{0} \in L_{\text {uloc }}^{2}\left(\mathbb{R}^{3}\right)$ with, in addition, $\left.u_{0}\right|_{B_{0}(2)} \in L^{6}\left(\mathbb{R}^{3}\right)$. Then, there exists a universal constant $K_{2} \in[1, \infty)$, there exists $u_{0, a} \in L_{\sigma}^{6}\left(\mathbb{R}^{3}\right) \cap L_{\sigma}^{2}\left(\mathbb{R}^{3}\right)$, $\operatorname{supp}\left(u_{0, a}\right) \subset$ $B_{0}(2)$, and $u_{0, b} \in L_{u l o c}^{2}\left(\mathbb{R}^{3}\right)$ such that the following holds:

$$
\begin{aligned}
& u_{0}=u_{0, a}+u_{0, b}, \quad u_{0, a}=u_{0} \text { on } B_{0}\left(\frac{3}{2}\right), \quad\left\|u_{0, a}\right\|_{L^{6}} \leq K_{2}\left\|u_{0}\right\|_{L^{6}\left(B_{0}(2)\right)}, \\
& \left\|u_{0, a}\right\|_{L^{2}} \leq K_{2}\left\|u_{0}\right\|_{L^{2}\left(B_{0}(2)\right)} \text { and }\left\|u_{0, b}\right\|_{L_{u l o c}^{2}} \leq K_{2}\left\|u_{0}\right\|_{L_{u l o c}^{2}} .
\end{aligned}
$$

Proof. The proof is standard using Bogovskii's operator [15, Chapter III.3]. We refer to [6] for a detailed proof.

Step 2: control of the local energy of the perturbation. We use the decomposition given by Lemma 5.6 for $u_{0}$ as above. Let $a$ be the mild solution given by Proposition A.1 associated to the data $u_{0, a} \in L^{6}\left(\mathbb{R}^{3}\right)$. The mild solution $a$ exists at least on the time interval $\left(0, S_{\text {mild }}^{a}\right)$, where

$$
S_{\text {mild }}^{a}:=k_{0} N^{-4} \text {. }
$$

Moreover since $u_{0} \in L_{\sigma}^{2}\left(\mathbb{R}^{3}\right)$, the mild solution $a$ can be constructed to be a weak Leray-Hopf solution on $\mathbb{R}^{3} \times\left(0, S_{\text {mild }}^{a}\right)$ and we have the global energy control ${ }^{38}$

$$
\sup _{s \in\left(0, S_{\text {mild }}^{a}\right)} \int_{\mathbb{R}^{3}} \frac{|a(x, s)|^{2}}{2} d x+\int_{0}^{S_{\text {mild }}^{a}} \int_{\mathbb{R}^{3}}|\nabla a|^{2} d x d s \leq K_{0}^{\prime} N^{2},
$$

with $K_{0}^{\prime} \in[1, \infty)$ a universal constant. This and Calderón-Zygmund theory implies

$$
\left\|q_{a}\right\|_{L^{\frac{5}{3}}\left(\mathbb{R}^{3} \times\left(0, S_{\text {mild }}^{a}\right)\right)} \leq K_{0}^{\prime \prime} N^{2},
$$

38 This can be inferred from arguments similar to [5, Section 3.1]. 
where $q_{a}$ is the pressure associated to $a$. Moreover, since $u$ is a local energy solution with the initial data $u_{0}$, Proposition A. 3 implies the following control of the local energy

$$
\sup _{s \in\left(0, S_{\text {locen }}^{u}\right)} \sup _{\bar{x} \in \mathbb{R}^{3}} \int_{B_{\bar{x}}(1)} \frac{|u(x, s)|^{2}}{2} d x+\sup _{\bar{x} \in \mathbb{R}^{3}} \int_{0}^{S_{\text {locen }}^{u}} \int_{B_{\bar{x}}(1)}|\nabla u(x, s)|^{2} d x d s \leq K_{1} M^{2}
$$

where $S_{\text {locen }}^{u}(N):=k_{1} \min \left(M^{-4}, 1\right)$. As a consequence, the perturbation $v=u-a$ is a local energy solution to (187)

$$
\begin{aligned}
& \sup _{s \in\left(0, S^{v}\right)} \sup _{\bar{x} \in \mathbb{R}^{3}} \int_{B_{\bar{x}}(1)} \frac{|v(x, s)|^{2}}{2} d x+\sup _{\bar{x} \in \mathbb{R}^{3}} \int_{0}^{S^{v}} \int_{B_{\bar{x}}(1)}|\nabla v(x, s)|^{2} d x d s \\
& \leq K_{1}^{\prime}\left(N^{2}+M^{2}\right),
\end{aligned}
$$

where $K_{1}^{\prime} \in[1, \infty)$ is a universal constant and

$$
\begin{aligned}
S^{v} & =S^{v}(M, N):=\min \left(\frac{1}{4}, S_{\text {mild }}^{a}, S_{\text {locen }}^{u}\right) \\
& =\min \left(\frac{1}{4}, k_{0} N^{-4}, k_{1} M^{-4}, k_{1}\right) .
\end{aligned}
$$

Moreover, we have the following pressure estimate

$$
\left\|q-C_{0}(t)\right\|_{L^{\frac{5}{3}}\left(B_{0}\left(\frac{3}{2}\right) \times\left(0, S^{v}\right)\right)} \leq K_{1}^{\prime}\left(M^{2}+N^{2}\right),
$$

with a universal constant $K_{1}^{\prime} \in[1, \infty)$. This bound follows from (198), (303) and (306).

Step 3: smallness of the local energy for a short time. Let $\phi \in C_{c}^{\infty}\left(\mathbb{R}^{3}\right)$ be a cut-off function such that

$$
0 \leq \phi \leq 1, \quad \operatorname{supp} \phi \subset B_{0}\left(\frac{3}{2}\right), \quad \phi=1 \text { on } B_{0}(1) \quad \text { and } \quad\left|\nabla\left(\phi^{2}\right)\right|+\left|\Delta\left(\phi^{2}\right)\right| \leq K_{3},
$$

where $K_{3} \in[1, \infty)$. We estimate the local energy

$$
E(t):=\sup _{s \in(0, t)} \int_{\mathbb{R}^{3}}|v(x, t)|^{2} \phi^{2} d x+2 \int_{0}^{t} \int_{\mathbb{R}^{3}}|\nabla v|^{2} \phi^{2} d x d s
$$

for all $t \in\left(0, S^{v}\right)$. The local energy inequality gives

$$
E(t) \leq I_{1}+\ldots I_{6}
$$

with

$$
\begin{aligned}
& I_{1}=\int_{0}^{t} \int_{\mathbb{R}^{3}}|v|^{2} \Delta\left(\phi^{2}\right) d x d s, \quad I_{2}=\int_{0}^{t} \int_{\mathbb{R}^{3}}|v|^{2} v \cdot \nabla\left(\phi^{2}\right) d x d s, \\
& I_{3}=2 \int_{0}^{t} \int_{\mathbb{R}^{3}}\left(q-C_{0}(t)\right) v \cdot \nabla\left(\phi^{2}\right) d x d s, \quad I_{4}=-2 \int_{0}^{t} \int_{\mathbb{R}^{3}}(a \cdot \nabla v) \cdot v \phi^{2} d x d s,
\end{aligned}
$$




$$
I_{5}=2 \int_{0}^{t} \int_{\mathbb{R}^{3}}(a \otimes v): \nabla v \phi^{2} d x d s \text { and } I_{6}=2 \int_{0}^{t} \int_{\mathbb{R}^{3}}(a \otimes v): v \otimes \nabla\left(\phi^{2}\right) d x d s
$$

Let $t \in\left(0, S^{v}\right)$. Let us estimate each term in the right hand side. For that purpose, we rely on the bounds (200) for the local energy and (202) for the pressure. For the terms involving only $v$, we have using that $\left|B_{0}\left(\frac{3}{2}\right)\right|=3^{3}$,

$$
\left|I_{1}\right| \leq 3^{3} K_{3} \cdot 2 K_{1}^{\prime}\left(M^{2}+N^{2}\right) t
$$

and

$$
\begin{aligned}
\left|I_{2}\right| & \leq 3^{3+\frac{3}{10}} K_{3}\left(3^{3} \cdot 2 K_{1}^{\prime}\right)^{\frac{3}{2}}\left(M^{2}+N^{2}\right)^{\frac{3}{2}} t^{\frac{1}{10}} \\
& =2^{\frac{3}{2}} \cdot 3^{\frac{39}{5}} K_{3} K_{1}^{\prime \frac{3}{2}}\left(M^{2}+N^{2}\right)^{\frac{3}{2}} t^{\frac{1}{10}}
\end{aligned}
$$

For the terms involving $a$ and $v$ we use (299) in Proposition A.1, more precisely the bound $\|a\|_{L^{10}\left(\mathbb{R}^{3} \times\left(0, S_{\text {mild }}^{a}\right)\right)} \leq K_{0} N$. This in turn implies the controls

$$
\begin{aligned}
\left|I_{4}+I_{5}+I_{6}\right| & \leq 3\|a\|_{L^{10}} 3^{3} E(t)\left(3^{3} t\right)^{\frac{1}{5}-\frac{1}{10}} \\
& \leq 2 \cdot 3 \frac{43}{10} K_{0} K_{3} K_{1}^{\prime} N\left(M^{2}+N^{2}\right) t^{\frac{1}{10}} .
\end{aligned}
$$

For $I_{6}$, we used $t \in\left(0, S^{v}\right) \subset\left(0, \frac{1}{4}\right)$.

Finally, we estimate the term involving the pressure

$$
\begin{aligned}
\left|I_{3}\right| & \leq 2 K_{3}\left\|q-C_{0}(t)\right\|_{L^{\frac{5}{3}}\left(B_{0}\left(\frac{3}{2}\right) \times(0, t)\right)}\|v\|_{L^{\frac{10}{3}}\left(B_{0}\left(\frac{3}{2}\right) \times(0, t)\right)}\left(3^{3} t\right)^{\frac{1}{10}} \\
& \leq 2 \cdot 3^{\frac{9}{5}} K_{3} K_{1}^{\prime \frac{3}{2}}\left(M^{2}+N^{2}\right)^{\frac{3}{2}} t^{\frac{1}{10}} .
\end{aligned}
$$

Finally, we get the following estimate: there exists a universal constant $K_{*} \in[1, \infty)$ such that for all $t \in(0,1]$,

$$
E(t) \leq K_{*}\left(M^{2}+N^{2}\right)^{\frac{3}{2}} t^{\frac{1}{10}}
$$

Notice that $K_{*}$ can be taken as

$$
K_{*}:=4 \max \left(2 \cdot 3^{3} K_{3} K_{1}^{\prime}, 2^{\frac{3}{2}} \cdot 3^{\frac{39}{5}} K_{3} K_{1}^{\prime \frac{3}{2}}, 2 \cdot 3^{\frac{43}{10}} K_{0} K_{1}^{\prime}\right),
$$

where $K_{0}$ is defined in Proposition A.1, $K_{1}^{\prime}$ in (200) and (202), and $K_{3}$ is the constant in (203).

Step 4: boundedness of the perturbation. Let $\varepsilon \in\left(0, \min \left(\varepsilon_{*}, 2^{-9}\right)\right)$. Our objective is now to apply the $\varepsilon$-regularity result Corollary 5.5 in order to get the boundedness of the perturbation. As in [20] and [6], we extend $v$ by 0 in the past. The extension $v$ is a suitable weak solution on $B_{0}(1) \times\left(-\infty, S^{v}\right)$ to the Navier-Stokes equations (187) with a drift $a$ defined to be the zero extension of $a$ to $\mathbb{R}^{3} \times(-\infty, 0)$. The bound on the local energy (204) is crucial here, as is emphasized in [20]. Notice that the extended $a$ is not a mild solution to the Navier-Stokes system (1) on $\mathbb{R}^{3} \times\left(-\infty, S_{\text {mild }}^{a}\right)$ but in Corollary 5.5 this fact is not required. We have the bound

$$
\|a\|_{L^{10}\left(\mathbb{R}^{3} \times\left(-\infty, S_{m i l d}^{a}\right)\right)} \leq K_{0} N .
$$


By the control (200) on the local energy and (202) on the pressure, we have

$$
\begin{aligned}
\int_{t-1}^{t} \int_{B_{0}(1)}|v|^{3}+\left|q-C_{0}(t)\right|^{\frac{3}{2}} d x d s & =\int_{0}^{t} \int_{B_{0}(1)}|v|^{3}+\left|q-C_{0}(t)\right|^{\frac{3}{2}} d x d s \\
& \leq 2 K_{1}^{\prime \frac{3}{2}}\left(M^{2}+N^{2}\right)^{\frac{3}{2}}\left(2^{3} t\right)^{\frac{1}{10}} .
\end{aligned}
$$

Therefore, in order to apply Corollary 5.5, we choose $S_{*}=S_{*}(M, N) \in\left(0, S^{v}\right)$ sufficiently small such that

$$
\begin{array}{r}
K_{*}\left(M^{2}+N^{2}\right)^{\frac{3}{2}} S_{*}^{\frac{1}{10}} \leq\left(K_{0} N\right)^{-2} \varepsilon^{\frac{11}{9}}, \\
2^{\frac{13}{10}} K_{1}^{\prime \frac{3}{2}}\left(M^{2}+N^{2}\right)^{\frac{3}{2}} S_{*}^{\frac{1}{10}} \leq\left(K_{0} N\right)^{-4} \varepsilon^{\frac{13}{9}} .
\end{array}
$$

Conditions (207) imply that (192) and (194) are satisfied on $B_{0}(1) \times\left(S_{*}-1, S_{*}\right)$. According to (207), we define $S_{*}=S_{*}(M, N)$ in the following way

$$
S_{*}:=\min \left(S^{v}, \frac{2^{-\frac{3}{2}} \varepsilon^{\frac{130}{9}}}{K_{0}^{40} M^{30} N^{70}} \min \left(\frac{1}{2^{13} K_{1}^{\prime 15}}, \frac{1}{K_{*}^{10}}\right)\right)
$$

Notice that for $M, N$ sufficiently large we have

$$
\begin{aligned}
S_{*} & =\frac{2^{-\frac{3}{2}} \varepsilon^{\frac{130}{9}}}{K_{0}^{40} M^{30} N^{70}} \min \left(\frac{1}{2^{13} K_{1}^{\prime 15}}, \frac{1}{K_{*}^{10}}\right) \\
& =O\left(M^{-30} N^{-70}\right) .
\end{aligned}
$$

For the rest of the proof we take this definition of $S_{*}$. It follows from (195) that

$$
\|u-a\|_{L^{\infty}\left(Q_{\left(0, S_{*}\right)}\left(\frac{1}{2}\right)\right)}=\|v\|_{L^{\infty}\left(Q_{\left(0, S_{*}\right)}\left(\frac{1}{2}\right)\right)} \leq C_{* * *}^{\frac{1}{3}} N^{2} .
$$

Combining this estimate with (299) enables us to obtain that for all $\beta \in\left(0, S_{*}\right)$

$$
\|u\|_{L^{\infty}\left(B_{0}\left(\frac{1}{2}\right) \times\left(\beta, S_{*}\right)\right)} \leq C_{* * *}^{\frac{1}{3}} N^{2}+\frac{K_{0} N}{\beta^{\frac{1}{4}}},
$$

which implies estimate (177) as a particular case.

Step 5: estimates of the gradient of the perturbation. In this step, we take $\beta=\frac{3 S_{*}}{4}$. Our goal is to prove the following claim

$$
\sup _{\bar{x} \in B_{0}\left(\frac{1}{4}\right)} \sup _{t \in\left(\frac{15}{16} S_{*}, S_{*}\right)} \int_{B_{\bar{x}}\left(\frac{\sqrt{S_{*}}}{4}\right)}|\nabla u(x, t)|^{2} d x \leq C M^{34} N^{80},
$$

for a universal constant $C \in[1, \infty)$. Estimate (212) implies estimate (178) in the theorem by a covering argument. Let $\bar{x} \in B_{0}\left(\frac{1}{4}\right)$. Notice that $B_{\bar{x}}\left(\frac{\sqrt{S_{*}}}{2}\right) \subset B_{0}\left(\frac{1}{3}\right)$ for $M, N$ sufficiently large. Without loss of generality, we assume that $\bar{x}=0$. We bootstrap the regularity of $u$ in the parabolic cylinder

$$
Q_{\left(0, S_{*}\right)}\left(\frac{\sqrt{S_{*}}}{2}\right)=B_{0}\left(\frac{\sqrt{S_{*}}}{2}\right) \times\left(\frac{3}{4} S_{*}, S_{*}\right)
$$


using the local maximal regularity for the non-stationary Stokes system. We first zoom in on the parabolic cylinder $Q_{\left(0, S_{*}\right)}\left(\frac{\sqrt{S_{*}}}{2}\right)$ and define

$$
U(y, s):=r u\left(r y, r^{2} s+S_{*}\right), \quad P(y, s):=r^{2}\left(p\left(r y, r^{2} s+S_{*}\right)-C_{0}\left(r^{2} s+S_{*}\right)\right),
$$

for all $(y, s) \in Q_{(0,0)}(1)$, where $r:=\frac{\sqrt{S_{*}}}{2}$. By the estimate (177), we have

$$
\begin{aligned}
\|U\|_{L^{\infty}\left(Q_{(0,0)}(1)\right)} & \leq \frac{C_{*}}{2} M^{8} N^{19} S_{*}^{\frac{1}{2}} \\
& \leq C M^{-7} N^{-16} .
\end{aligned}
$$

Moreover, the local energy estimate (199) implies that

$$
\|\nabla U\|_{L^{2}\left(Q_{(0,0)}(1)\right)}=r^{-\frac{1}{2}}\|\nabla u\|_{L^{2}\left(Q_{\left(0, S_{*}\right)}(r)\right)} \leq 2^{\frac{1}{2}} K_{1}^{\frac{1}{2}} M S_{*}^{-\frac{1}{4}} \leq C M^{9} N^{18},
$$

For the pressure, we decompose $p-C_{0}(t)$ according to (303). We have according to the estimates in Proposition A.3

$$
\begin{aligned}
\left\|p_{\text {nonloc }}\right\|_{L^{2}\left(Q_{\left(0, S_{*}\right)}(r)\right)} & \leq\left\|p_{\text {nonloc }}\right\|_{L^{2}\left(B_{0}\left(\frac{1}{2}\right) \times\left(\frac{3}{4} S_{*}, S_{*}\right)\right)} \\
& \leq S_{*}^{\frac{1}{2}}\left\|p_{\text {nonloc }}\right\|_{L^{\infty}\left(B_{0}\left(\frac{1}{2}\right) \times\left(\frac{3}{4} S_{*}, S_{*}\right)\right)} \\
& \leq S_{*}^{\frac{1}{2}} K_{1} M^{2},
\end{aligned}
$$

on the one hand, and

$$
\begin{aligned}
\left\|p_{\text {loc }}\right\|_{L^{2}\left(Q_{\left(0, S_{*}\right)}(r)\right)} & \leq\left\|p_{\text {loc }}\right\|_{L^{2}\left(B_{0}\left(\frac{1}{2}\right) \times\left(\frac{3}{4} S_{*}, S_{*}\right)\right)} \\
& \leq C\|u\|_{L^{4}\left(B_{0}\left(\frac{1}{2}\right) \times\left(\frac{3}{4} S_{*}, S_{*}\right)\right)}^{2} \\
& \leq C S_{*}^{\frac{1}{2}}\|u\|_{L^{\infty}\left(B_{0}\left(\frac{1}{2}\right) \times\left(\frac{3}{4} S_{*}, S_{*}\right)\right)}^{2} \\
& \leq C S_{*}^{\frac{1}{2}} M^{16} N^{39}
\end{aligned}
$$

using (304) and Calderón-Zygmund on the other hand. Hence,

$$
\begin{aligned}
\|P\|_{L^{2}\left(Q_{(0,0)}(1)\right)} & =r^{-\frac{1}{2}}\|p-C(t)\|_{L^{2}\left(Q_{\left(0, S_{*}\right)}(r)\right)} \leq 2^{\frac{1}{2}} S_{*}^{-\frac{1}{4}}\left(S_{*}^{\frac{1}{2}} K_{1} M^{2}+C S_{*}^{\frac{1}{2}} M^{16} N^{39}\right) \\
& \leq C M^{9} N^{22} .
\end{aligned}
$$

Notice that these are rough bounds, but they are enough for our purposes. Therefore,

$$
\|\nabla U\|_{L^{2}\left(Q_{(0,0)}(1)\right)}+\|P\|_{L^{2}\left(Q_{(0,0)}(1)\right)} \leq C M^{9} N^{22} .
$$

Using the local maximal regularity for the non-stationary Stokes system [41, Proposition 2.4] leads to

$$
\begin{aligned}
& \left\|\partial_{t} U\right\|_{L^{2}\left(Q_{(0,0)}\left(\frac{3}{4}\right)\right)}+\left\|\nabla^{2} U\right\|_{L^{2}\left(Q_{(0,0)}\left(\frac{3}{4}\right)\right)}+\|\nabla P\|_{L^{2}\left(Q_{(0,0)}\left(\frac{3}{4}\right)\right)} \\
& \leq C\left(\|U\|_{L^{\infty}\left(Q_{(0,0)}(1)\right)}\|\nabla U\|_{L^{2}\left(Q_{(0,0)}(1)\right)}\right. \\
& \left.\quad+\|U\|_{L^{2}\left(Q_{(0,0)}(1)\right)}+\|\nabla U\|_{L^{2}\left(Q_{(0,0)}(1)\right)}+\|P\|_{L^{2}\left(Q_{(0,0)}(1)\right)}\right)
\end{aligned}
$$




$$
\leq C M^{9} N^{22}
$$

where we used the bounds (213) and (214). A simple local energy estimate for $\nabla U$ then leads to the bound

$$
\|\nabla U\|_{L_{t}^{\infty} L_{x}^{2}\left(Q_{(0,0)}\left(\frac{1}{2}\right)\right)} \leq C M^{9} N^{22} .
$$

Scaling back to the original variables leads to (212) and concludes the proof.

\section{Main Tool 2: Quantitative Annuli of Regularity}

In this section we prove that a solution $u$, satisfying the hypothesis of Propositions 2.12.2 , enjoys good quantitative bounds in certain spatial annuli. This was crucially used in the aforementioned propositions in two places. Namely for applying the Carleman inequalities (Propositions B.1-B.2), as well as in 'Step 4' for transferring the lower bound of the vorticity to a lower bound on the localized $L^{3}$ norm.

In the context of classical solutions to the Navier-Stokes equations in $L_{t}^{\infty} L_{x}^{3}\left(\mathbb{R}^{3} \times\right.$ $\left.\left(t_{0}-T, t_{0}\right)\right)$, a related version was proven by Tao in [47] using a delicate analysis of local enstrophies from [46]. Our proof is somewhat different and elementary (though we use the 'pigeonhole principle' as in [47]), instead we utilize known $\varepsilon$-regularity criteria. Notice that a crucial point in the following statement is that the $L^{\infty}$ bound is quantified in terms of $\varepsilon_{0}$, see below.

Proposition 6.1 ( [9] and [25, Theorem 30.1]). There exists absolute constants $\varepsilon_{0}^{*}>0$ and $C_{C K N} \in(0, \infty)$ such that if $(u, p)$ is a suitable weak solution to the Navier-Stokes equations on $Q_{(0,0)}(1)$ and for some $\varepsilon_{0} \leq \varepsilon_{0}^{*}$

$$
\int_{Q_{(0,0)}(1)}|u|^{3}+|p|^{\frac{3}{2}} d x d t \leq \varepsilon_{0}
$$

then one concludes that

$$
u \in L^{\infty}\left(Q_{(0,0)}(1 / 2)\right) \text { with }\|u\|_{L^{\infty}\left(Q_{(0,0)}\left(\frac{1}{2}\right)\right)} \leq C_{C K N} \varepsilon_{0}^{\frac{1}{3}} .
$$

We require the following proposition, which is a quantitative version of Serrin's result [42]. Since the procedure is the same as that described in [42], we omit the proof.

Proposition 6.2 Suppose $u \in L^{\infty}\left(Q_{(0,0)}(1 / 2)\right)$ and $\omega:=\nabla \times u \in L^{2}\left(Q_{(0,0)}(1 / 2)\right)$ is such that $(u, p)$ is a distributional solution to the Navier-Stokes equations in $Q_{(0,0)}(1 / 2)$. Furthermore, suppose

$$
\|u\|_{L^{\infty}\left(Q_{(0,0)}(1 / 2)\right)}<1
$$

and

$$
\|\omega\|_{L^{2}\left(Q_{(0,0)}(1 / 2)\right)}<1 .
$$

There exists universal constants $C_{k}^{\prime} \in(0, \infty)$ with $k=0,1$, such that the above assumptions imply that for $k=0,1$

$$
\left\|\nabla^{k} \omega\right\|_{L^{\infty}\left(Q_{(0,0)}(1 / 3)\right)} \leq C_{k}^{\prime}\left(\|u\|_{L^{\infty}\left(Q_{(0,0)}(1 / 2)\right)}+\|\omega\|_{L^{2}\left(Q_{(0,0)}(1 / 2)\right)}\right) .
$$


Remark 6.3. If we instead use the framework of suitable weak solutions in the above proposition, we can use the time integrability of the pressure to gain space-time Hölder continuity of all spatial derivatives of $u$ in $Q_{(0,0)}(1 / 3)$. The vorticity equation then implies $w, \partial_{t} w, \nabla w$ and $\nabla^{2} w$ are continuous in space and time in $Q_{(0,0)}(1 / 3)$.

By using Proposition 6.2, one can recover the following well-known proposition whose proof we omit.

Proposition 6.4 There exists absolute constants $\varepsilon_{1}^{*} \in(0,1)$ and $C_{k}^{\prime \prime} \in(0, \infty), k=$ $0,1,2$, such that if $(u, p)$ is a suitable weak solution to the Navier-Stokes equations on $Q_{(0,0)}(1)$ and for some $\varepsilon_{1} \leq \varepsilon_{1}^{*}$

$$
\int_{Q_{(0,0)}(1)}|u|^{3}+|p|^{\frac{3}{2}} d x d t \leq \varepsilon_{1}
$$

then one concludes that for $j=0,1$

$$
\nabla^{j} u \in L^{\infty}\left(Q_{(0,0)}(1 / 4)\right) \text { with }\left\|\nabla^{j} u\right\|_{L^{\infty}\left(Q_{(0,0)}\left(\frac{1}{4}\right)\right)} \leq C_{j}^{\prime \prime} \varepsilon_{1}^{\frac{1}{3}}
$$

and

$$
\nabla \omega \in L^{\infty}\left(Q_{(0,0)}(1 / 4)\right) \text { with }\|\nabla \omega\|_{L^{\infty}\left(Q_{(0,0)}\left(\frac{1}{4}\right)\right)} \leq C_{2}^{\prime \prime} \epsilon_{1}^{\frac{1}{3}} .
$$

Proposition 6.5 (annulus of regularity, general form). For all $\mu>0$ there exists $\lambda_{0}(\mu)>$ 1 such that the following holds true. For all $\lambda \geq \lambda_{0}(\mu), R \geq 1$ and for every solution $(u, p)$ to the Navier-Stokes equations on $\mathbb{R}^{3} \times[-1,0]$ that is a suitable weak solution on $Q_{\left(x_{*}, 0\right)}(1)$ for all $x_{*} \in \mathbb{R}^{3}$ and satisfies

$$
\int_{-1}^{0} \int_{\mathbb{R}^{3}}|u|^{\frac{10}{3}}+|p|^{\frac{5}{3}} d x d t \leq \lambda<\infty
$$

there exists $R^{\prime \prime}(u, p, \lambda, \mu, R)$ with

$$
2 R \leq R^{\prime \prime} \leq 2 R \exp \left(2 \mu \lambda^{\mu+2}\right)
$$

and universal constants $\bar{C}_{j} \in(0, \infty)$ for $j=0,1,2$ such that for $j=0,1$

$$
\left\|\nabla^{j} u\right\|_{L^{\infty}\left(\left\{R^{\prime \prime}<|x|<\frac{\lambda^{\mu}}{4} R^{\prime \prime}\right\} \times\left(-\frac{1}{16}, 0\right)\right)} \leq \bar{C}_{j} \lambda^{-\frac{3 \mu}{10}}
$$

and

$$
\|\nabla \omega\|_{L^{\infty}\left(\left\{R^{\prime \prime}<|x|<\frac{\lambda^{\mu}}{4} R^{\prime \prime}\right\} \times\left(-\frac{1}{16}, 0\right)\right)} \leq \bar{C}_{2} \lambda^{-\frac{3 \mu}{10}} .
$$

Proof. Fix any $R \geq 1$ and $\mu>0$. With these choices, take $\lambda>\lambda_{0}(\mu) \geq 1$. Here, $\lambda_{0}$ is to be determined. Then

$$
\sum_{k=0}^{\infty} \int_{-1}^{0} \int_{\left(\lambda^{\mu}\right)^{k} R<|x|<\left(\lambda^{\mu}\right)^{k+1} R}|u|^{\frac{10}{3}}+|p|^{\frac{5}{3}} d x d t \leq \lambda .
$$


By the pigeonhole principle, there exists $k_{0} \in\left\{0,1, \ldots\left\lceil\lambda^{\mu+1}\right\rceil\right\}$ such that

$$
\int_{-1}^{0} \int_{\left(\lambda^{\mu}\right)^{k_{0}}}|u|_{R<|x|<\left(\lambda^{\mu}\right)^{k_{0}+1} R}^{\frac{10}{3}}+|p|^{\frac{5}{3}} d x d t \leq \lambda^{-\mu} .
$$

Define $R^{\prime}:=R \lambda^{\mu k_{0}}$. Then

$$
R \leq R^{\prime} \leq R \exp \left(2 \mu \lambda^{\mu+2}\right)
$$

and

$$
\int_{-1}^{0} \int_{R^{\prime}<|x|<\lambda^{\mu} R^{\prime}}|u|^{\frac{10}{3}}+|p|^{\frac{5}{3}} d x d t \leq \lambda^{-\mu}
$$

Impose the restriction $\lambda_{0}(\mu)>4^{\frac{1}{\mu}}$ and define

$$
A:=\left\{x: R^{\prime}+1<|x|<\lambda^{\mu} R^{\prime}-1\right\} .
$$

By Hölder's inequality we have

$$
\begin{aligned}
\sup _{x_{*} \in A} \int_{-1}^{0} \int_{B\left(x_{*}, 1\right)}|u|^{3}+|p|^{\frac{3}{2}} d x d t & \leq C_{\text {univ }}\left(\sup _{x_{*} \in A} \int_{-1}^{0} \int_{B_{x_{*}}(1)}|u|^{\frac{10}{3}}+|p|^{\frac{5}{3}} d x d t\right)^{\frac{9}{10}} \\
& \leq C_{\text {univ }} \lambda^{-\frac{9 \mu}{10}}
\end{aligned}
$$

Defining

$$
\lambda_{0}(\mu):=\max \left(2 \cdot 4^{\frac{1}{\mu}},\left(\frac{2 C_{u n i v}}{\varepsilon_{1}^{*}}\right)^{\frac{10}{9 \mu}}\right),
$$

the inequality $\lambda \geq \lambda_{0}(\mu)$ implies that

$$
\sup _{x_{*} \in A} \int_{-1}^{0} \int_{B_{x_{*}}(1)}|u|^{3}+|p|^{\frac{3}{2}} d x d t \leq C_{\text {univ }} \lambda^{-\frac{9 \mu}{10}}<\varepsilon_{1}^{*} \text {. }
$$

Thus, we can apply Proposition 6.4 to get that for $j=0,1$

$$
\sup _{x_{*} \in A}\left\|\nabla^{j} u\right\|_{L^{\infty}\left(Q_{\left(x_{*}, 0\right)}\left(\frac{1}{4}\right)\right)} \leq C_{j}^{\prime \prime} C_{u n i v}^{\frac{1}{3}} \lambda^{-\frac{3 \mu}{10}} \text { and } \sup _{x_{*} \in A}\|\nabla \omega\|_{L^{\infty}\left(Q_{\left(x_{*}, 0\right)}\left(\frac{1}{4}\right)\right)} \leq C_{2}^{\prime \prime} C_{u n i v}^{\frac{1}{3}} \lambda^{-\frac{3 \mu}{10}} .
$$

Hence,

$$
\left\|\nabla^{j} u\right\|_{L^{\infty}\left(A \times\left(-\frac{1}{16}, 0\right)\right)} \leq C_{j}^{\prime \prime} C_{u n i v}^{\frac{1}{3}} \lambda^{-\frac{3 \mu}{10}} \text { and }\|\nabla \omega\|_{L^{\infty}\left(A \times\left(-\frac{1}{16}, 0\right)\right)} \leq C_{2}^{\prime \prime} C_{u n i v}^{\frac{1}{3}} \lambda^{-\frac{3 \mu}{10}} .
$$

Finally, note that (230) and $\lambda \geq \lambda_{0}$ imply that $\lambda^{\mu} R^{\prime}-1>\frac{\lambda^{\mu}}{2} R^{\prime}>2 R^{\prime}>R^{\prime}+1$. Defining $R^{\prime \prime}:=2 R^{\prime}$ and using $\left\{x: R^{\prime \prime}<|x|<\frac{\lambda^{\mu}}{4} R^{\prime \prime}\right\} \subset A$, we see that the above estimates readily give the desired conclusion. 
Bearing in mind (265), the energy estimates (264), (270) and Calderón-Zygmund estimates for the pressure, the following Lemma is obtained as an immediate corollary to the above Proposition. Note that (265), (264) and (270) are shown in the proof of Lemma 7.3. We also use the known fact that mild solutions to the Navier-Stokes equations in $L_{x, t}^{4}\left(\mathbb{R}^{3} \times(0, T)\right)$ are suitable weak solutions on $\mathbb{R}^{3} \times(0, T)$, which can be seen by using a mollification argument along with Calderón-Zygmund estimates for the pressure.

Lemma 6.6 (annulus of regularity, Type I). For all $\mu>0$ there exist $M_{1}(\mu), c(\mu), C(\mu)$ $>1$ such that the following holds true. For all $M \geq M_{1}(\mu), R \geq 1$ and for every mild solution $(u, p)$ of the Navier-Stokes equations on $\mathbb{R}^{3} \times[-2,0]$ satisfying

$$
\|u\|_{L_{t}^{\infty} L_{x}^{3, \infty}\left(\mathbb{R}^{3} \times(-2,0)\right)} \leq M
$$

and

$$
u \in L_{x, t}^{4}\left(\mathbb{R}^{3} \times(-2,0)\right),
$$

there exists $R^{\prime \prime}(u, p, M, \mu, R)$ with

$$
2 R \leq R^{\prime \prime} \leq 2 R \exp \left(C(\mu) M^{10(\mu+2)}\right)
$$

and universal constants $\bar{C}_{j} \in(0, \infty)$ for $j=0,1,2$ such that for $j=0,1$

$$
\left\|\nabla^{j} u\right\|_{L^{\infty}\left(\left\{R^{\prime \prime}<|x|<c(\mu) M^{10 \mu} R^{\prime \prime}\right\} \times\left(-\frac{1}{16}, 0\right)\right)} \leq \bar{C}_{j} C(\mu) M^{-3 \mu}
$$

and

$$
\|\nabla \omega\|_{L^{\infty}\left(\left\{R^{\prime \prime}<|x|<c(\mu) M^{10 \mu} R^{\prime \prime}\right\} \times\left(-\frac{1}{16}, 0\right)\right)} \leq \bar{C}_{2} C(\mu) M^{-3 \mu} .
$$

A simple rescaling gives the following corollary, which is directly used in the proof of Proposition 2.1.

Corollary 6.7 Let $S \in(0, \infty)$. For all $\mu>0$, let $M_{1}(\mu), c(\mu), C(\mu)>1$ be given by Lemma 6.6. For all $M \geq M_{1}(\mu), R \geq S^{\frac{1}{2}}$ and for every mild solution $(u, p)$ of the Navier-Stokes equations on $\mathbb{R}^{3} \times[-S, 0]$ satisfying

$$
\|u\|_{L_{t}^{\infty} L_{x}^{3, \infty}\left(\mathbb{R}^{3} \times(-S, 0)\right)} \leq M
$$

and

$$
u \in L_{x, t}^{4}\left(\mathbb{R}^{3} \times(-S, 0)\right),
$$

there exists $R^{\prime \prime}(u, p, M, \mu, R)$ with (233) and universal constants $\bar{C}_{j}$ for $j=0,1,2$ such that for $j=0,1$

$$
\left\|\nabla^{j} u\right\|_{L^{\infty}\left(\left\{R^{\prime \prime}<|x|<c(\mu) M^{10 \mu} R^{\prime \prime}\right\} \times\left(-\frac{S}{32}, 0\right)\right)} \leq 2^{\frac{j+1}{2}} \bar{C}_{j} C(\mu) M^{-3 \mu} S^{-\frac{j+1}{2}}
$$

and

$$
\|\nabla \omega\|_{L^{\infty}\left(\left\{R^{\prime \prime}<|x|<c(\mu) M^{10 \mu} R^{\prime \prime}\right\} \times\left(-\frac{S}{32}, 0\right)\right)} \leq 2^{\frac{2}{3}} \bar{C}_{2} C(\mu) M^{-3 \mu} S^{-\frac{3}{2}} .
$$

Bearing in mind (293), the energy estimate in footnote 12 (see also Lemma 3.3) and Calderón-Zygmund estimates for the pressure, the following Lemma is obtained as an immediate corollary to Proposition 6.5. 
Lemma 6.8 (annulus of regularity, time slices). For all $\mu>0$ there exists $M_{2}(\mu)>1$ such that the following holds true. For all $M \geq M_{2}(\mu), R \geq 1$ and for every suitable finite-energy solution $^{39}(u, p)$ of the Navier-Stokes equations on $\mathbb{R}^{3} \times[-1,0]$ satisfying

$$
\|u(\cdot,-1)\|_{L^{3}\left(\mathbb{R}^{3}\right)} \leq M
$$

and with $M^{b}$ defined by (17), there exists $R^{\prime \prime}(u, p, M, \mu, R)$ with

$$
2 R \leq R^{\prime \prime} \leq 2 R \exp \left(C(\mu)\left(M^{b}\right)^{10(\mu+2)}\right)
$$

and universal constants $\bar{C}_{j}$ for $j=0,1,2$ such that for $j=0,1$

$$
\left\|\nabla^{j} u\right\|_{L^{\infty}\left(\left\{R^{\prime \prime}<|x|<\left(M^{\mathrm{b}}\right)^{10 \mu} R^{\prime \prime}\right\} \times\left(-\frac{1}{16}, 0\right)\right)} \leq \bar{C}_{j}\left(M^{\mathrm{b}}\right)^{-3 \mu}
$$

and

$$
\|\nabla \omega\|_{L^{\infty}\left(\left\{R^{\prime \prime}<|x|<\left(M^{b}\right)^{10 \mu} R^{\prime \prime}\right\} \times\left(-\frac{1}{16}, 0\right)\right)} \leq \bar{C}_{2}\left(M^{b}\right)^{-3 \mu} .
$$

A simple rescaling gives the following corollary, which is directly used in the proof of Theorem B.

Corollary 6.9 Let $S \in(0, \infty)$. For all $\mu>0$, let $M_{2}(\mu)>1$ and $M^{b}$ be given by Lemma 6.8. For all $M \geq M_{2}(\mu), R \geq S^{\frac{1}{2}}$ and for every suitable finite-energy solution $(u, p)$ of the Navier-Stokes equations on $\mathbb{R}^{3} \times[-S, 0]$ satisfying

$$
\|u(\cdot,-S)\|_{L^{3}\left(\mathbb{R}^{3}\right)} \leq M,
$$

there exists $R^{\prime \prime}(u, p, M, \mu, R)$ with

$$
2 R \leq R^{\prime \prime} \leq 2 R \exp \left(C(\mu)\left(M^{b}\right)^{10(\mu+2)}\right)
$$

and universal constants $\bar{C}_{j}$ for $j=0,1,2$ such that for $j=0,1$

$$
\left\|\nabla^{j} u\right\|_{L^{\infty}\left(\left\{R^{\prime \prime}<|x|<\left(M^{\mathrm{b}}\right)^{10 \mu} R^{\prime \prime}\right\} \times\left(-\frac{S}{32}, 0\right)\right)} \leq 2^{\frac{j+1}{2}} \bar{C}_{j}\left(M^{\mathrm{b}}\right)^{-3 \mu} S^{-\frac{j+1}{2}}
$$

and

$$
\|\nabla \omega\|_{L^{\infty}\left(\left\{R^{\prime \prime}<|x|<\left(M^{\mathrm{b}}\right)^{10 \mu} R^{\prime \prime}\right\} \times\left(-\frac{S}{32}, 0\right)\right)} \leq 2^{\frac{3}{2}} \bar{C}_{2}\left(M^{b}\right)^{-3 \mu} S^{-\frac{3}{2}} .
$$

Remark 6.10. According to Remark 6.3, in Proposition 6.5-Corollary 6.9 we have that $w, \partial_{t} w, \nabla w$ and $\nabla^{2} w$ are continuous in space and time on the annuli considered. This remark is needed to apply the first Carleman inequality, Proposition B.1, in Sects. 3 and 4 .

39 See Sect. 1.4 'Notations' for a definition of 'suitable finite-energy solutions.' 


\section{Main Tool 3: Quantitative Epochs of Regularity}

In this section, we prove that a solution satisfying the hypothesis of Propositions 2.1-2.2 enjoys good quantitative estimates in certain time intervals. In the literature, these are commonly referred to as 'epochs of regularity'. Such a property is crucially used in 'Step 1 ' of the above propositions, when applying a quantitative Carleman inequality based on unique continuation (Proposition B.2).

To show the existence of such epochs of regularity, we follow Leray's approach in [27]. In particular, we utilize arguments involving existence of mild solutions for subcritical data and weak-strong uniqueness. We provide full details for the reader's convenience.

We first recall a result known as 'O'Neil's convolution inequality' (Theorem 2.6 of O'Neil's paper [33]).

Proposition 7.1 Suppose $1<p_{1}, p_{2}, r<\infty$ and $1 \leq q_{1}, q_{2}, s \leq \infty$ are such that

$$
\frac{1}{r}+1=\frac{1}{p_{1}}+\frac{1}{p_{2}}
$$

and

$$
\frac{1}{q_{1}}+\frac{1}{q_{2}} \geq \frac{1}{s}
$$

Suppose that

$$
f \in L^{p_{1}, q_{1}}\left(\mathbb{R}^{d}\right) \text { and } g \in L^{p_{2}, q_{2}}\left(\mathbb{R}^{d}\right) .
$$

Then

$$
\begin{aligned}
& f * g \in L^{r, s}\left(\mathbb{R}^{d}\right) \text { with } \\
& \|f * g\|_{L^{r, s}\left(\mathbb{R}^{d}\right)} \leq 3 r\|f\|_{L^{p_{1}, q_{1}}\left(\mathbb{R}^{d}\right)}\|g\|_{L^{p_{2}, q_{2}}\left(\mathbb{R}^{d}\right)} .
\end{aligned}
$$

We will also use an inequality that we will refer to as 'Hölder's inequality for Lorentz spaces'. The statement below and its proof can be found in [18, Theorem 4.5, p.271]; see also [33, Theorems 3.4-3.5, page 141].

Proposition 7.2 Suppose that $1 \leq p, q, r \leq \infty$ and $1 \leq s_{1}, s_{2} \leq \infty$. Let $\Omega$ be a domain in $\mathbb{R}^{d}$. Furthermore, suppose that $p, q, r, s_{1}$ and $s_{2}$ satisfy the following relations:

$$
\frac{1}{p}+\frac{1}{q}=\frac{1}{r}
$$

and

$$
\frac{1}{s_{1}}+\frac{1}{s_{2}}=\frac{1}{s} .
$$

Then the assumption that $f \in L^{p, s_{1}}(\Omega)$ and $g \in L^{q, s_{2}}(\Omega)$ implies that $f g \in L^{r, s}(\Omega)$, with the estimate

$$
\|f g\|_{L^{r, s}(\Omega)} \leq C\left(p, q, s_{1}, s_{2}\right)\|f\|_{L^{p, s_{1}}(\Omega)}\|g\|_{L^{q, s_{2}}(\Omega)} .
$$

Below we apply Proposition 7.1 and Proposition 7.2 to obtain a quantitative epoch of regularity for a solution to the Navier-Stokes equations with Type I bound, let us mention that the application of such inequalities to the Navier-Stokes equations was pioneered by Kozono and Yamazaki [22,23,52]. 
Lemma 7.3 (epoch of regularity, Type I). There exists a universal constant $C_{3} \in[1, \infty)$ such that the following holds. Suppose $u: \mathbb{R}^{3} \times\left[t_{0}-T, t_{0}\right] \rightarrow \mathbb{R}^{3}$ is a mild solution ${ }^{40}$ of the Navier-Stokes equations. Furthermore, assume for some $M \geq 1$ that

$$
\|u\|_{L_{t}^{\infty} L_{x}^{3, \infty}\left(\mathbb{R}^{3} \times\left(t_{0}-T, t_{0}\right)\right)} \leq M
$$

and

$$
u \in L_{x, t}^{4}\left(\mathbb{R}^{3} \times\left(t_{0}-T, t_{0}\right)\right) .
$$

Then for all intervals $I \subset\left[t_{0}-\frac{T}{2}, t_{0}\right]$ there exists a subinterval $I^{\prime} \subset I$ such that the following holds true. Namely,

$$
\left\|\nabla^{j} u\right\|_{L_{t}^{\infty} L_{x}^{\infty}\left(\mathbb{R}^{3} \times I^{\prime}\right)} \leq C_{3} M^{18}|I|^{\frac{-(j+1)}{2}}
$$

for $j=0,1,2$ and

$$
\left|I^{\prime}\right| \geq C_{3}^{-1} M^{-12}|I|
$$

Remark 7.4 (estimates for applying Carleman inequalities (Type I)). Let $I^{\prime \prime} \subset I^{\prime}$ be such that

$$
\left|I^{\prime \prime}\right|=\frac{M^{-36}}{4 C_{3}^{2}}\left|I^{\prime}\right| .
$$

Then

$$
\left|I^{\prime}\right|^{-1}=\frac{M^{-36}}{4 C_{3}^{2}}\left|I^{\prime \prime}\right|^{-1} .
$$

Using (254)-(256), together with the fact that $C_{3}$ and $M \in[1, \infty)$, we see that

$$
\left\|\nabla^{j} u\right\|_{L_{t}^{\infty} L_{x}^{\infty}\left(\mathbb{R}^{3} \times I^{\prime \prime}\right)} \leq \frac{1}{2^{j+1}}\left|I^{\prime \prime}\right|^{\frac{-(j+1)}{2}}
$$

for $j=0,1,2$ and

$$
\left|I^{\prime \prime}\right| \geq \frac{M^{-48}}{4 C_{3}^{3}}|I| .
$$

Proof. The first part of the proof closely follows arguments in Tao's paper [47]. The only difference in the first part of the proof is that we exploit the above facts regarding Lorentz spaces. For completeness, we give full details.

As observed by Tao in [47], (254)-(257) are invariant with respect to the NavierStokes scaling and time translation. So we can assume without loss of generality

$$
I=[0,1] \subset\left[t_{0}-\frac{T}{2}, t_{0}\right] \Rightarrow[-1,1] \subset\left[t_{0}-T, t_{0}\right]
$$

Step 1: a priori energy estimates. Clearly we have from the standing assumptions that

$$
\|u\|_{L_{t}^{\infty} L_{x}^{3, \infty}\left(\mathbb{R}^{3} \times(-1,1)\right)} \leq M .
$$

\footnotetext{
40 See Sect. 1.4.4.
} 
On $\mathbb{R}^{3} \times(-1,1)$ we have

$$
u=e^{(t+1) \Delta} u(\cdot,-1)+w, \quad w:=-\int_{-1}^{t} e^{(t-s) \Delta} \mathbb{P} \nabla \cdot(u \otimes u)(\cdot, s) d s .
$$

It is known that $e^{t \Delta} \mathbb{P} \nabla$. has an associated convolution kernel $K$. Furthermore, from Solonnikov's paper [44], this satisfies the estimate

$$
\left|\partial_{t}^{m} \nabla^{j} K(x, t)\right| \leq \frac{C(m, j)}{\left(|x|^{2}+t\right)^{2+\frac{j}{2}+m}}, \text { for } j, m=0,1 \ldots
$$

Thus we may apply O'Neil's convolution inequality (Proposition 7.1) with $r=s=2$, $q_{1}=p_{1}=\frac{6}{5}, p_{2}=\frac{3}{2}$ and $q_{2}=\infty$. This and Hölder's inequality for Lorentz spaces (Proposition 7.2) gives that for $t \in[-1,1]$

$$
\|w(\cdot, t)\|_{L_{x}^{2}} \leq C \int_{-1}^{t} \frac{\|u \otimes u(\cdot, s)\|_{L^{\frac{3}{2}, \infty}}}{(t-s)^{\frac{3}{4}}} d s \leq C M^{2}(t+1)^{\frac{1}{4}} .
$$

Thus,

$$
\|w\|_{L_{t}^{\infty} L_{x}^{2}\left(\mathbb{R}^{3} \times(-1,1)\right)} \leq C M^{2}
$$

Using O'Neil's convolution inequality once more gives

$$
\begin{aligned}
& \left\|e^{(t+1) \Delta} u(\cdot,-1)\right\|_{L_{x}^{\frac{10}{3}}} \leq \frac{C M}{(t+1)^{\frac{1}{20}}}, \\
& \left\|e^{(t+1) \Delta} u(\cdot,-1)\right\|_{L_{x}^{4}} \leq \frac{C M}{(t+1)^{\frac{1}{8}}},
\end{aligned}
$$

and

$$
\left\|e^{(t+1) \Delta} u(\cdot,-1)\right\|_{L_{x}^{6,2}} \leq \frac{C M}{(t+1)^{\frac{1}{4}}} .
$$

By utilizing (266) for $\frac{1}{2}+\frac{t}{2}$ instead of $1+t$, together with standard heat semigroup estimates, one can also obtain

$$
\left\|e^{(t+1) \Delta} u(\cdot,-1)\right\|_{L_{x}^{\infty}} \leq \frac{C M}{(t+1)^{\frac{1}{2}}} .
$$

Next, we see that $w$ satisfies

$$
\partial_{t} w-\Delta w+u \cdot \nabla u+\nabla p=0, \quad \nabla \cdot w=0 \quad w(\cdot,-1)=0 .
$$

Using (255), (268) and (266) we infer the following. Namely, $w \in C\left([0,1] ; L_{\sigma}^{2}\left(\mathbb{R}^{3}\right)\right) \cap$ $L^{2}\left(0,1 ; \dot{H}^{1}\left(\mathbb{R}^{3}\right)\right)$ and satisfies the energy equality ${ }^{41}$ for $t \in[0,1]$ :

$$
\frac{1}{2}\|w(\cdot, t)\|_{L_{x}^{2}}^{2}+\int_{0}^{t} \int_{\mathbb{R}^{3}}|\nabla w|^{2} d x d t^{\prime}
$$

\footnotetext{
41 This can be shown by utilizing arguments in [26] (Lemma 7.2 in [26]) and [43] (Theorem 2.3.1 in [43]), for example.
} 


$$
=\frac{1}{2}\|w(\cdot, 0)\|_{L_{x}^{2}}^{2}+\int_{0}^{t} \int_{\mathbb{R}^{3}} e^{\left(t^{\prime}+1\right) \Delta} u(\cdot,-1) \otimes\left(w+e^{\left(t^{\prime}+1\right) \Delta} u(\cdot,-1)\right): \nabla w d x d t^{\prime}
$$

Using Hölder's inequality followed by Young's inequality we see that

$$
\begin{aligned}
\|w(\cdot, t)\|_{L_{x}^{2}}^{2}+\int_{0}^{t} \int_{\mathbb{R}^{3}}|\nabla w|^{2} d x d t^{\prime} \leq & \|w(\cdot, 0)\|_{L_{x}^{2}}^{2}+C \int_{0}^{t} \int_{\mathbb{R}^{3}}\left|e^{\left(t^{\prime}+1\right) \Delta} u(\cdot,-1)\right|^{4} d x d t^{\prime} \\
& +C \int_{0}^{t}\left\|w\left(\cdot, t^{\prime}\right)\right\|_{L_{x}^{2}}^{2}\left\|e^{\left(t^{\prime}+1\right) \Delta} u(\cdot,-1)\right\|_{L_{x}^{\infty}}^{2} d t^{\prime} .
\end{aligned}
$$

Using this, together with (264)-(266) and the fact $M>1$, we obtain

$$
\int_{0}^{1} \int_{\mathbb{R}^{3}}|\nabla w|^{2} d x d t^{\prime} \leq C_{\text {univ }} M^{6}
$$

Here, $C_{\text {univ }}$ is a universal constant.

Step 2: higher integrability via weak-strong uniqueness. From (270), the pigeonhole principle, the Sobolev embedding theorem and (267), there exists $t_{1} \in\left[0, \frac{1}{2}\right]$ such that

$$
\left\|u\left(\cdot, t_{1}\right)\right\|_{L^{6}\left(\mathbb{R}^{3}\right)} \leq C_{\text {univ }} M^{3} .
$$

This, (254) and the interpolation inequality for the Lorentz spaces (see Lemma 2.2 in [30] for example) implies that $u\left(\cdot, t_{1}\right) \in L_{\sigma}^{4}\left(\mathbb{R}^{3}\right)$. This and (271) allows us to apply Proposition A.1 and Remark A.2. In particular, there exists $C_{\text {univ }}^{\prime} \in(0, \infty)$ and a mild solution $U: \mathbb{R}^{3} \times\left[t_{1}, t_{1}+\frac{C_{\text {univ }}^{\prime}}{M^{12}}\right] \rightarrow \mathbb{R}^{3}$ to the Navier-Stokes equations, with initial data $u\left(\cdot, t_{1}\right)$, which satisfies the following properties. Specifically,

$$
\|U\|_{L_{t}^{\infty} L_{x}^{6}\left(\mathbb{R}^{3} \times\left[t_{1}, t_{1}+\frac{C_{\text {univ }}^{\prime}}{M^{12}}\right]\right)} \leq C M^{3}
$$

and

$$
U \in L_{t}^{\infty} L_{x}^{4}\left(\mathbb{R}^{3} \times\left[t_{1}, t_{1}+\frac{C_{u n i v}^{\prime}}{M^{12}}\right]\right) .
$$

Let $W: \mathbb{R}^{3} \times\left[t_{1}, t_{1}+\frac{C_{u n i v}^{\prime}}{M^{12}}\right] \rightarrow \mathbb{R}^{3}$ be defined by

$$
W:=u-U=-\int_{t_{1}}^{t} e^{(t-s) \Delta} \mathbb{P} \nabla \cdot(u \otimes u-U \otimes U)(\cdot, s) d s \text { for } t \in\left[t_{1}, t_{1}+\frac{C_{u n i v}^{\prime}}{M^{12}}\right]
$$

Using (255) and (273), we see that

$$
W \in C\left(\left[t_{1}, t_{1}+\frac{C_{\text {univ }}^{\prime}}{M^{12}}\right] ; L_{\sigma}^{2}\left(\mathbb{R}^{3}\right)\right) \cap L_{t}^{2}\left(t_{1}, t_{1}+\frac{C_{u n i v}^{\prime}}{M^{12}} ; \dot{H}^{1}\left(\mathbb{R}^{3}\right)\right)
$$


and $W$ satisfies the energy equality for $t \in\left[t_{1}, t_{1}+\frac{C_{\text {univ }}^{\prime}}{M^{12}}\right]$ :

$$
\|W(\cdot, t)\|_{L_{x}^{2}}^{2}+2 \int_{t_{1}}^{t} \int_{\mathbb{R}^{3}}|\nabla W|^{2} d x d t^{\prime}=2 \int_{t_{1}}^{t} \int_{\mathbb{R}^{3}} U \otimes W: \nabla W d x d t^{\prime} .
$$

Using this, (272) and known weak-strong uniqueness arguments from [27], we infer that $W \equiv 0$ on $\mathbb{R}^{3} \times\left[t_{1}, t_{1}+\frac{C_{u n i v}^{\prime}}{M^{12}}\right]$. Using this together with (272), we get that for $\tau(s):=t_{1}+\frac{s C_{u n i v}^{\prime}}{M^{12}}:$

$$
\|u\|_{L_{t}^{\infty} L_{x}^{6}\left(\mathbb{R}^{3} \times(\tau(0), \tau(1))\right)} \leq C M^{3} .
$$

Using that the pressure is given by a Riesz transform acting on $u \otimes u$, we can apply Calderón-Zygmund to get that the pressure $p$ associated to $u$ satisfies

$$
\|p\|_{L_{t}^{\infty} L_{x}^{3}\left(\mathbb{R}^{3} \times(\tau(0), \tau(1))\right)} \leq C M^{6} .
$$

Step 3: higher derivative estimates. Here, the arguments differ from those utilized in [47]. Fix any $x \in \mathbb{R}^{3}$ and $t \in\left[\tau\left(\frac{1}{2}\right), \tau(1)\right]$. Take any $r \in\left(0, \sqrt{\frac{C_{\text {univ }}^{\prime}}{2 M^{12}}}\right]$, which ensures that $t-r^{2} \in[\tau(0), \tau(1)]$. Using this and (277)-(278) we see that

$$
\frac{1}{r^{2}} \int_{Q_{(x, t)}(r)}|u|^{3}+|p|^{\frac{3}{2}} d x d t^{\prime} \leq C_{u n i v}^{\prime \prime} r^{\frac{3}{2}} M^{9}=C_{u n i v}^{\prime \prime}\left(r M^{6}\right)^{\frac{3}{2}} .
$$

Taking

$$
r=r_{0}:=\frac{1}{M^{6}} \min \left(\sqrt{\frac{C_{\text {univ }}^{\prime}}{2}}, \frac{\epsilon_{C K N}^{\frac{2}{3}}}{\left(C_{\text {univ }}^{\prime \prime}\right)^{\frac{2}{3}}}\right),
$$

where $\epsilon_{C K N}$ is $\varepsilon_{0}^{*}$ in [38, Lemma 6.1], we can then apply the Caffarelli-Kohn-Nirenberg theorem [9] and [38, Lemma 6.1] to get that for $j=0,1, \ldots$

$$
\sup _{(x, t) \in \mathbb{R}^{3} \times\left[\tau\left(\frac{1}{2}\right), \tau(1)\right]}\left|\nabla^{j} u(x, t)\right| \leq \frac{C}{r_{0}^{j+1}} \simeq C(j)\left(M^{6}\right)^{j+1} .
$$

This concludes the proof.

Lemma 7.5 (epoch of regularity, time slices). There exists a universal constant $C_{4} \in$ $[1, \infty)$ such that the following holds. Suppose $u:[-1,0] \times \mathbb{R}^{3} \rightarrow \mathbb{R}^{3}$ and $p$ : $[-1,0] \times \mathbb{R}^{3} \rightarrow \mathbb{R}$ is a suitable finite-energy solution to the Navier-Stokes equations. Furthermore, assume for some $M \geq 1$ and $t_{0} \in[-1,0)$ that

$$
\left\|u\left(\cdot, t_{0}\right)\right\|_{L_{x}^{3}} \leq M
$$

and $u$ satisfies the energy inequality (43) starting from $t^{\prime}=t_{0}$.

We define $M^{b}$ as in (17). Fix any $\alpha \geq M^{b}$ and let

$$
s_{0} \in\left[\frac{t_{0}}{2}, \frac{t_{0}}{4 \alpha^{201}}\right] \text {. }
$$


Define

$$
I:=\left[s_{0}, \frac{s_{0}}{2}\right] .
$$

There exists a subinterval $I^{\prime} \subset$ I such that the following holds true. Namely,

$$
\left\|\nabla^{j} u\right\|_{L_{t}^{\infty} L_{x}^{\infty}\left(\mathbb{R}^{3} \times I^{\prime}\right)} \leq C_{4} \alpha^{324}|I|^{\frac{-(j+1)}{2}}
$$

for $j=0,1,2$ and

$$
\left|I^{\prime}\right| \geq C_{4}^{-1} \alpha^{-216}|I|
$$

Remark 7.6 (estimates for applying Carleman inequalities, time slices). Let $I^{\prime \prime} \subset I^{\prime}$ be such that

$$
\left|I^{\prime \prime}\right|=\frac{\alpha^{-648}}{4 C_{4}^{2}}\left|I^{\prime}\right|
$$

Then

$$
\left|I^{\prime}\right|^{-1}=\frac{\alpha^{-648}}{4 C_{4}^{2}}\left|I^{\prime \prime}\right|^{-1} .
$$

Using (283)-(284), together with the fact that $C_{4}$ and $M \in[1, \infty)$, we see that

$$
\left\|\nabla^{j} u\right\|_{L_{t}^{\infty} L_{x}^{\infty}\left(\mathbb{R}^{3} \times I^{\prime \prime}\right)} \leq \frac{1}{2^{j+1}}\left|I^{\prime \prime}\right|^{\frac{-(j+1)}{2}}
$$

for $j=0,1,2$ and

$$
\left|I^{\prime \prime}\right| \geq \frac{\alpha^{-864}}{4 C_{4}^{3}}|I| .
$$

Proof. Define,

$$
\hat{t}:=\frac{t_{0}}{s_{0}}-1
$$

Note that (281) implies that

$$
\hat{t} \in\left(1,4 \alpha^{201}-1\right) .
$$

By appropriate scalings and translations, we can assume without loss of generality that $u: \mathbb{R}^{3} \times(0, \hat{T}) \rightarrow \mathbb{R}^{3}$, for some $\hat{T} \in(0, \infty)^{42}$ and

$$
\begin{aligned}
& \|u(\cdot, 0)\|_{L^{3}\left(\mathbb{R}^{3}\right)} \leq M, \\
& \|u(\cdot, t)\|_{L^{2}}^{2}+2 \int_{0}^{t} \int_{\mathbb{R}^{3}}|\nabla u(y, s)|^{2} d y d s \leq\|u(\cdot, 0)\|_{L^{2}}^{2}
\end{aligned}
$$

and

$$
I:=\left[\hat{t}, \hat{t}+\frac{1}{2}\right] \subset(1, \hat{T})
$$

42 The time $\hat{T}$ is the image of 0 by the scalings and translations. Its precise value does not matter at all, since the proof is carried out on the time interval $\left[0, \hat{t}+\frac{1}{2}\right]$. 
On $\mathbb{R}^{3} \times(0, \hat{T})$ we have

$$
u=e^{t \Delta} u(\cdot, 0)+w .
$$

Using O'Neil's convolution inequality once more gives for all $t \in(0, \infty)$,

$$
\begin{aligned}
& \left\|e^{t \Delta} u(\cdot, 0)\right\|_{L_{x}^{3}} \leq C M, \\
& \left\|e^{t \Delta} u(\cdot, 0)\right\|_{L_{x}^{\frac{10}{3}}} \leq \frac{C M}{t^{\frac{1}{20}}}, \\
& \left\|e^{t \Delta} u(\cdot, 0)\right\|_{L_{x}^{4}} \leq \frac{C M}{t^{\frac{1}{8}}},
\end{aligned}
$$

and

$$
\left\|e^{t \Delta} u(\cdot, 0)\right\|_{L_{x}^{6,2}} \leq \frac{C M}{t^{\frac{1}{4}}} .
$$

Furthermore, arguments from [17] imply that

$$
\left\|e^{t \Delta} u(\cdot, 0)\right\|_{L^{5}\left(\mathbb{R}^{3} \times(0, \infty)\right)} \leq C M .
$$

Moreover, similar arguments as those used in Proposition 2.2 of [40] yield that for $t \in(0, \hat{T})$,

$$
\begin{aligned}
& \|w(\cdot, t)\|_{L_{x}^{2}}^{2}+\int_{0}^{t} \int_{\mathbb{R}^{3}}|\nabla w|^{2} d x d t^{\prime} \\
& \leq C \int_{0}^{t} \int_{\mathbb{R}^{3}}\left|e^{t \Delta} u(\cdot, 0)\right|^{4} d x d t^{\prime}+C \int_{0}^{t}\left\|w\left(\cdot, t^{\prime}\right)\right\|_{L_{x}^{2}}^{2}\left\|e^{t^{\prime} \Delta} u(\cdot, 0)\right\|_{L_{x}^{5}}^{5} d t^{\prime} .
\end{aligned}
$$

Note that the energy inequality for $w$, which is used to produce this estimate, can be justified rigorously using (296) and similar arguments as those used in Proposition 14.3 in [25].

Using (292)-(296) and Gronwall's lemma, we infer that

$$
\sup _{0<t<\hat{t}+\frac{1}{2}}\|w(\cdot, t)\|_{L_{x}^{2}}^{2}+\int_{0}^{\hat{t}+\frac{1}{2}} \int_{\mathbb{R}^{3}}|\nabla w|^{2} d x d t^{\prime} \leq\left(M^{b}\right)^{4}\left(\hat{t}+\frac{1}{2}\right)^{\frac{1}{2}}<2 \alpha^{105} .
$$

Here we used (287). Now let $\Sigma \subset[0, \hat{T}]$ be such that (43) is satisfied for all $t \in\left[t^{\prime}, \hat{T}\right]$ and $t^{\prime} \in \Sigma$. Since $u$ is a suitable finite-energy solution we have that $|\Sigma|=\hat{T}$. Furthermore, $\Sigma$ can be chosen without loss of generality such that

$$
\int_{\mathbb{R}^{3}}\left|\nabla w\left(x, t^{\prime}\right)\right|^{2} d x<\infty \text { for all } t^{\prime} \in \Sigma .
$$

Using (297), the Sobolev embedding theorem, the pigeonhole principle and (295), we see that there exists $t_{1} \in\left[\hat{t}, \hat{t}+\frac{1}{4}\right] \cap \Sigma$ such that

$$
\left\|u\left(\cdot, t_{1}\right)\right\|_{L^{6}}^{2} \leq C \alpha^{105} .
$$

Making use of the fact that $u$ satisfies the energy inequality starting from $t_{1}$ and (298), we can utilize similar arguments to those used in Lemma 7.3 replacing $M$ by $\alpha^{18}$. 
Acknowledgement. The authors acknowledge stimulating discussions with Dallas Albritton about Terence Tao's paper [47] and for suggesting Leray's approach for showing existence of epochs of regularity. The authors also thank Jan Burczak for discussions about Type I singularities, and Dongho Chae and Jörg Wolf for bringing to our attention the paper [10]. Finally, the authors express their gratitude to the referees for their helpful comments.

Funding The first author is supported by a Leverhulme Early Career Fellowship funded by The Leverhulme Trust. The second author is partially supported by the project BORDS grant ANR-16-CE40-0027-01 and by the project Sing Flows grant ANR-18-CE40-0027 of the French National Research Agency (ANR). The second author also acknowledges financial support from the IDEX of the University of Bordeaux for the BOLIDE project.

Open Access This article is licensed under a Creative Commons Attribution 4.0 International License, which permits use, sharing, adaptation, distribution and reproduction in any medium or format, as long as you give appropriate credit to the original author(s) and the source, provide a link to the Creative Commons licence, and indicate if changes were made. The images or other third party material in this article are included in the article's Creative Commons licence, unless indicated otherwise in a credit line to the material. If material is not included in the article's Creative Commons licence and your intended use is not permitted by statutory regulation or exceeds the permitted use, you will need to obtain permission directly from the copyright holder. To view a copy of this licence, visit http://creativecommons.org/licenses/by/4.0/.

Publisher's Note Springer Nature remains neutral with regard to jurisdictional claims in published maps and institutional affiliations.

\section{Declarations}

Conflict of interest The authors declare that they have no conflict of interest.

\section{Appendix A: Auxiliary Results}

We first state the existence result of mild solutions with subcritical data.

Proposition A.1 ( $[17,51])$. Let $n \in(3, \infty)$. There exists $k_{0}(n) \in(0, \infty), K_{0}(n) \in$ $[1, \infty)$ such that the following holds. For all $u_{0} \in L_{\sigma}^{n}\left(\mathbb{R}^{3}\right)$, we define

$$
S_{\text {mild }}\left(u_{0}\right):=k_{0}\left\|u_{0}\right\|_{L^{n}}^{-\frac{2 n}{n-3}} \in(0, \infty) .
$$

There exists a unique mild solution of $(1), a \in C\left(\left[0, S_{\text {mild }}\right) ; L^{n}\right) \cap L^{\infty}\left(\left(0, S_{\text {mild }}\right) ; L^{n}\right)$, with initial data $u_{0}$ such that

$$
\begin{aligned}
& \sup _{t \in(0, S}\left(\|a(\cdot, t)\|_{L^{n}}+t^{\frac{3}{2 n}}\|a(\cdot, t)\|_{L^{\infty}}+t^{\frac{1}{2}}\|\nabla a(\cdot, t)\|_{L^{n}}+t^{\frac{1}{2}+\frac{3}{2 n}}\|\nabla a(\cdot, t)\|_{L^{\infty}}\right) \\
& \in\left(0, S_{\text {mild }}\right) \\
& +\|a\|_{L^{\frac{5 n}{3}}\left(\mathbb{R}^{3} \times\left(0, S_{\text {mild }}\right)\right)} \leq K_{0}\left\|u_{0}\right\|_{L^{n}} .
\end{aligned}
$$

Remark A.2. Here $n=6$. For $U, V: \mathbb{R}^{3} \times(0, T) \rightarrow \mathbb{R}^{3}$ define

$$
B(U, V)(\cdot, t):=\int_{0}^{t} \mathbb{P} \partial_{i} e^{(t-s) \Delta} U_{i}(\cdot, s) V_{j}(\cdot, s) d s .
$$

Using (263), one has the estimate

$$
\begin{array}{r}
\|B(U, V)\|_{L_{t}^{\infty} L_{x}^{4}\left(\mathbb{R}^{3} \times[0, T]\right)}+\|B(V, U)\|_{L_{t}^{\infty} L_{x}^{4}\left(\mathbb{R}^{3} \times[0, T]\right)} \\
\leq c T^{\frac{1}{4}}\|U\|_{L_{t}^{\infty} L_{x}^{6}\left(\mathbb{R}^{3} \times[0, T]\right)}\|V\|_{L_{t}^{\infty} L_{x}^{4}\left(\mathbb{R}^{3} \times[0, T]\right)} .
\end{array}
$$


Using this, one can show that for $u_{0} \in L_{\sigma}^{6}\left(\mathbb{R}^{3}\right) \cap L_{\sigma}^{4}\left(\mathbb{R}^{3}\right)$ and $k_{0}$ sufficiently small the following persistency property holds true. Namely, with $n=6$ the mild solution in Proposition A.1 satisfies

$$
\sup _{t \in\left(0, S_{\text {mild }}\right)}\|a(\cdot, t)\|_{L^{4}} \leq 2\left\|u_{0}\right\|_{L^{4}}
$$

in addition to (299). This is utilized in the proof of Lemma 7.3.

The next result is the local energy bound for local energy solutions. ${ }^{43}$

Proposition A.3 ( [19, Lemma 2.1], [25]). There exist two universal constants $k_{1} \in$ $(0, \infty), K_{1} \in[1, \infty)$ such that the following holds. For all $M \in(0, \infty)$, we define

$$
S_{\text {locen }}(M):=k_{1} \min \left(M^{-4}, 1\right) \in(0, \infty) .
$$

For all $u_{0} \in L_{\text {uloc }}^{2}\left(\mathbb{R}^{3}\right)$ with $\left\|u_{0}\right\|_{L^{2}\left(B_{\bar{x}}(1)\right)} \stackrel{|\bar{x}| \rightarrow \infty}{\longrightarrow} 0$, for all local energy solution $(u, p)$ to (1) with initial data $u_{0}$, if

$$
\sup _{\bar{x} \in \mathbb{R}^{3}} \int_{B_{\bar{x}}(1)}\left|u_{0}(x)\right|^{2} d x \leq M^{2},
$$

then

$$
\sup _{s \in\left(0, S_{\text {locen }}\right)} \sup _{\bar{x} \in \mathbb{R}^{3}} \int_{B_{\bar{x}}(1)} \frac{|u(x, s)|^{2}}{2} d x+\sup _{\bar{x} \in \mathbb{R}^{3}} \int_{0}^{S_{\text {locen }}} \int_{B_{\bar{x}}(1)}|\nabla u(x, s)|^{2} d x d s \leq K_{1} M^{2}
$$

Moreover, we have the following decomposition of the pressure: for all $\bar{x} \in \mathbb{R}^{3}$ and $t \in\left(0, S_{\text {locen }}\right)$, there exists $C_{\bar{x}}(t) \in \mathbb{R}$ such that ${ }^{44}$

$$
p(x, t)-C_{\bar{x}}(t)=-\frac{1}{3}|u(x, t)|^{2}+p_{l o c}(x, t)+p_{\text {nonloc }}(x, t)
$$

for all $(x, t) \in B_{\bar{x}}\left(\frac{3}{2}\right) \times\left(0, S_{\text {locen }}\right)$, with

$$
p_{l o c}(x, t)=-\int_{\mathbb{R}^{3}} K_{i j}(x-y) \varphi(y) u_{i}(y, t) u_{j}(y, t) d y
$$

and

$$
p_{\text {nonloc }}(x, t)=-\int_{\mathbb{R}^{3}}\left(K_{i j}(x-y)-K_{i j}(\bar{x}-y)\right)(1-\varphi(y)) u_{i}(y, t) u_{j}(y, t) d y .
$$

Here, $\varphi \in C_{0}^{\infty}\left(B_{\bar{x}}(4)\right)\left(\right.$ with $\varphi \equiv 1$ on $\left.B_{\bar{x}}(3)\right)$ and $K_{i j}(x):=\partial_{i} \partial_{j}\left(\frac{1}{|x|}\right)$.

Moreover, we have the estimate

$$
\left\|p_{\text {loc }}\right\|_{L^{\frac{5}{3}}\left(B_{\bar{x}}\left(\frac{3}{2}\right) \times\left(0, S_{\text {locen }}\right)\right)}+\left\|p_{\text {nonloc }}\right\|_{L^{\infty}\left(B_{\bar{x}}\left(\frac{3}{2}\right) \times\left(0, S_{\text {locen }}\right)\right)} \leq K_{1} M^{2} .
$$

\footnotetext{
${ }^{43}$ For a definition of 'local energy solutions', see Sect. 1.4.

44 This decomposition is also valid for $p_{\text {loc }}$ defined in $B_{\bar{x}}\left(\frac{1}{2}\right) \times\left(0, S_{\text {locen }}\right)$ instead of $B_{\bar{x}}\left(\frac{3}{2}\right) \times\left(0, S_{\text {locen }}\right)$ as stated here. The constant $C_{\bar{x}}(t)$ has to be adapted.
} 


\section{Appendix B: Carleman Inequalities}

The two statements below are taken directly from [47]. The first Carleman inequality in Proposition B.1 corresponds to a quantitative backward uniqueness result.

Proposition B.1 (first Carleman inequality [47, Proposition 4.2]). Let $C_{\text {Carl }} \in[1, \infty)$, $S \in(0, \infty), 0<r_{-}<r_{+}$and we define the space-time annulus

$$
\mathcal{A}:=\left\{(x, t) \in \mathbb{R}^{3} \times \mathbb{R}: t \in[0, S], r_{-} \leq|x| \leq r_{+}\right\} .
$$

Let $w: \mathcal{A} \rightarrow \mathbb{R}^{3}$ be such that $w, \partial_{t} w, \nabla w$ and $\nabla^{2} w$ are continuous in space and time and such that $w$ satisfies the differential inequality

$$
\left|\left(\partial_{t}+\Delta\right) w\right| \leq C_{\text {Carl }}^{-1} S^{-1}|w|+C_{C a r l}^{-\frac{1}{2}} S^{-\frac{1}{2}}|\nabla w| \text { on } \mathcal{A} .
$$

Assume

$$
r_{-}^{2} \geq 4 C_{\text {Carl }} S
$$

Then we have the following bound

$$
\int_{0}^{\frac{S}{4}} \int_{10 r_{-} \leq|x| \leq \frac{r_{+}}{2}}\left(S^{-1}|w|^{2}+|\nabla w|^{2}\right) d x d t \lesssim C_{\text {Carl }}^{3} e^{-\frac{r_{-} \cdot r_{+}}{4 C_{C a r l}}}\left(X+e^{\frac{2 r_{+}^{2}}{C_{\text {Carl }} S} Y}\right),
$$

where

$$
X:=\iint_{\mathcal{A}} e^{\frac{2|x|^{2}}{C_{C a r l} S}}\left(S^{-1}|w|^{2}+|\nabla w|^{2}\right) d x d t, \quad Y:=\int_{r_{-} \leq|x| \leq r_{+}}|w(x, 0)|^{2} d x .
$$

The second Carleman inequality in Proposition B.2 below corresponds to a quantitative unique continuation result.

Proposition B.2 (second Carleman inequality [47, Proposition 4.3]). Let $C_{\text {Carl }} \in$ $[1, \infty), S \in(0, \infty), r>0$ and we define the space-time cylinder

$$
\mathcal{C}:=\left\{(x, t) \in \mathbb{R}^{3} \times \mathbb{R}: t \in[0, S],|x| \leq r\right\} .
$$

Let $w: \mathcal{C} \rightarrow \mathbb{R}^{3}$ such that $w, \partial_{t} w, \nabla w$ and $\nabla^{2} w$ are continuous in space and time and such that $w$ satisfies the differential inequality

$$
\left|\left(\partial_{t}+\Delta\right) w\right| \leq C_{\text {Carl }}^{-1} S^{-1}|w|+C_{\text {Carl }}^{-\frac{1}{2}} S^{-\frac{1}{2}}|\nabla w| \text { on } \mathcal{C} .
$$

Assume

$$
r^{2} \geq 4000 S
$$

Then, for all $0<\underline{s} \leq \bar{s}<\frac{S}{10000}$ one has the bound

$$
\int_{\bar{s}}^{2 \bar{s}} \int_{|x| \leq \frac{r}{2}}\left(S^{-1}|w|^{2}+|\nabla w|^{2}\right) e^{-\frac{|x|^{2}}{4 t}} d x d t \lesssim e^{-\frac{r^{2}}{500 \bar{s}}} X+(\bar{s})^{\frac{3}{2}}\left(\frac{e \bar{s}}{\underline{s}}\right)^{\frac{C r^{2}}{\bar{s}}} Y
$$

where

$$
X:=\int_{0}^{S} \int_{|x| \leq r}\left(S^{-1}|w|^{2}+|\nabla w|^{2}\right) d x d t, \quad Y:=\int_{|x| \leq r}|w(x, 0)|^{2}(\underline{s})^{-\frac{3}{2}} e^{-\frac{|x|^{2}}{4 \underline{s}}} d x .
$$


Propositions B.1 and B.2 are proved in [47] for smooth functions. The proof works under the weaker smoothness assumption stated here. This is used in Sect. 4 in particular, where the results are stated for suitable finite-energy solutions.

\section{References}

1. Albritton, D.: Blow-up criteria for the Navier-Stokes equations in non-endpoint critical Besov spaces. Anal. PDE 11(6), 1415-1456 (2018)

2. Albritton, D., Barker, T.: Global weak Besov solutions of the Navier-Stokes equations and applications. Arch. Ration. Mech. Anal. 232(1), 197-263 (2019)

3. Albritton, D., Barker, T.: Localised necessary conditions for singularity formation in the Navier-Stokes equations with curved boundary. J. Differential Equations 269(9), 7529-7573 (2020)

4. Barker, T.: Uniqueness results for viscous incompressible fluids. PhD thesis, University of Oxford, (2017)

5. Barker, T.: Uniqueness results for weak Leray-Hopf solutions of the Navier-Stokes system with initial values in critical spaces. J. Math. Fluid Mech. 20(1), 133-160 (2018)

6. Barker, T., Prange, C.: Localized Smoothing for the Navier-Stokes Equations and Concentration of Critical Norms Near Singularities. Arch. Ration. Mech. Anal. 236(3), 1487-1541 (2020)

7. Barker, T., Seregin, G.: A necessary condition of potential blowup for the Navier-Stokes system in half-space. Math. Ann. 369(3-4), 1327-1352 (2017)

8. Bradshaw, Z., Tsai, T.-P.: Global existence, regularity, and uniqueness of infinite energy solutions to the Navier-Stokes equations. Commun. Part. Differ. Equ. 45(9), 1168-1201 (2020)

9. Caffarelli, L., Kohn, R., Nirenberg, L.: Partial regularity of suitable weak solutions of the Navier-Stokes equations. Commun. Pure Appl. Math. 35(6), 771-831 (1982)

10. Chae, D., Wolf, J.: Removing discretely self-similar singularities for the $3 \mathrm{~d}$ Navier-Stokes equations. Commun. Part. Differ. Equ. 42(9), 1359-1374 (2017)

11. Cheskidov, A., Shvydkoy, R.: The regularity of weak solutions of the $3 \mathrm{~d}$ Navier-Stokes equations in $B_{\infty, \infty}^{-1}$. Arch. Ration. Mech. Anal. 195(1), 159-169 (2010)

12. Choe, H., Wolf, J., Yang, M.: On regularity and singularity for $L^{\infty}\left(0, t ; L^{3, w}\left(R^{3}\right)\right)$ solutions to the navier-stokes equations. Math. Ann. (2019)

13. Escauriaza, L., Seregin, G. A., Šverák, V.: $L_{3, \infty}$-solutions of Navier-Stokes equations and backward uniqueness. Uspekhi Mat. Nauk, 58(2(350)):3-44, (2003)

14. Fefferman, C.L.: Existence and smoothness of the Navier-Stokes equation. Millenn. Prize Problem 57, 67 (2006)

15. Galdi, G.P.: An introduction to the mathematical theory of the Navier-Stokes equations. Springer Monographs in Mathematics. Springer, New York, second edition, 2011. Steady-state problems

16. Gallagher, I., Koch, G.S., Planchon, F.: Blow-up of critical Besov norms at a potential Navier-Stokes singularity. Commun. Math. Phys. 343(1), 39-82 (2016)

17. Giga, Y.: Solutions for semilinear parabolic equations in $L^{p}$ and regularity of weak solutions of the Navier-Stokes system. J. Differ, Equ. 62(2), 186-212 (1986)

18. Hunt, R.A.: On $L(p, q)$ spaces. Enseignement Math. 2(12), 249-276 (1966)

19. Jia, H., Šverák, V.: Minimal $L^{3}$-initial data for potential Navier-Stokes singularities. SIAM J. Math. Anal. 45(3), 1448-1459 (2013)

20. Jia, H., Šverák, V.: Local-in-space estimates near initial time for weak solutions of the Navier-Stokes equations and forward self-similar solutions. Invent. Math. 196(1), 233-265 (2014)

21. Kang, K., Miura, H., Tsai, T.-P.: Short Time Regularity of Navier-Stokes Flows with Locally $L^{3}$ Initial Data and Applications. Int. Math. Res. Not. 01, (2020)

22. Kozono, H., Yamazaki, M.: Exterior problem for the stationary Navier-Stokes equations in the Lorentz space. Math. Ann. 310(2), 279-305 (1998)

23. Kozono, H., Yamazaki, M.: Uniqueness criterion of weak solutions to the stationary Navier-Stokes equations in exterior domains. Nonlinear Anal., 38(8, Ser. A: Theory Methods):959-970, (1999)

24. Ladyzhenskaya, O.A.: On the uniqueness and on the smoothness of weak solutions of the Navier-Stokes equations. Zapiski Nauchnykh Seminarov POMI 5, 169-185 (1967)

25. Lemarié-Rieusset, P.G.: Recent developments in the Navier-Stokes problem. Chapman \&amp; Hall/CRC Research Notes in Mathematics, vol. 431. Chapman \& Hall/CRC, Boca Raton, FL (2002)

26. Lemarié-Rieusset, P.G.: The Navier-Stokes problem in the 21 st century. CRC Press (2016)

27. Leray, J.: Sur le mouvement d'un liquide visqueux emplissant l'espace. Acta Math. 63(1), 193-248 (1934)

28. Li, K., Ozawa, T., Wang, B.: Dynamical behavior for the solutions of the Navier-Stokes equation. Commun. Pure Appl. Anal. 17(4), 1511-1560 (2018) 
29. Maekawa, Y., Miura, H., Prange, C.: Local energy weak solutions for the Navier-Stokes equations in the half-space. Commun. Math. Phys. 367(2), 517-580 (2019)

30. McCormick, D.S., Robinson, J.C., Rodrigo, J.L.: Generalised Gagliardo-Nirenberg inequalities using weak lebesgue spaces and BMO. Milan J. Math. 81(2), 265-289 (2013)

31. Montgomery-Smith, S.: Conditions implying regularity of the three dimensional Navier-Stokes equation. Appl. Math. 50(5), 451-464 (2005)

32. Nečas, J., Ružička, M., Šverák, V.: On Leray's self-similar solutions of the Navier-Stokes equations. Acta Math. 176(2), 283-294 (1996)

33. O'Neil, R.: Convolution operators and $L(p, q)$ spaces. Duke Math. J., 30(1):129-142, 03 (1963)

34. Pan, X.: Regularity of solutions to axisymmetric Navier-Stokes equations with a slightly supercritical condition. J. Differ. Equ. 260(12), 8485-8529 (2016)

35. Phuc, N.C.: The Navier-Stokes equations in nonendpoint borderline Lorentz spaces. J. Math. Fluid Mech. 17(4), 741-760 (2015)

36. Rusin, W., Šverák, V.: Minimal initial data for potential Navier-Stokes singularities. J. Funct. Anal. 260(3), 879-891 (2011)

37. Seregin, G.: A certain necessary condition of potential blow up for Navier-Stokes equations. Commun. Math. Phys. 312(3), 833-845 (2012)

38. Seregin, G.: Lecture notes on regularity theory for the Navier-Stokes equations. World Scientific, Singapore (2014)

39. Seregin, G.: A note on weak solutions to the Navier-Stokes equations that are locally in $L_{\infty}\left(L^{3, \infty}\right)$, (2019)

40. Seregin, G., Šverák, V.: On global weak solutions to the Cauchy problem for the Navier-Stokes equations with large $L_{3}$-initial data. Nonlinear Anal. 154, 269-296 (2017)

41. Seregin, G.A.: A new version of the Ladyzhenskaya-Prodi-Serrin condition. Algebra I Analiz 18(1), 124-143 (2006)

42. Serrin, J.: On the interior regularity of weak solutions of the Navier-Stokes equations. Arch. Ration. Mech. Anal. 9(1), 187-195 (1962)

43. Sohr, H.: The Navier-Stokes equations: an elementary functional analytic approach. Springer, New York (2001)

44. Solonnikov, V.A.: Estimates for solutions of a non-stationary linearized system of Navier-Stokes equations. Trudy Matematicheskogo Instituta imeni VA Steklova 70, 213-317 (1964)

45. Struwe, M.: On partial regularity results for the Navier-Stokes equations. Commun. Pure Appl. Math. 41(4), 437-458 (1988)

46. Tao, T.: Localisation and compactness properties of the Navier-Stokes global regularity problem. Anal. PDE 6(1), 25-107 (2013)

47. Tao, T.: Quantitative bounds for critically bounded solutions to the Navier-Stokes equations. arXiv e-prints, arXiv:1908.04958, (2019)

48. Tsai, T.-P.: On Leray's self-similar solutions of the Navier-Stokes equations satisfying local energy estimates. Arch. Ration. Mech. Anal. 143(1), 29-51 (1998)

49. Tsai, T.-P.: Forward discretely self-similar solutions of the Navier-Stokes equations. Commun. Math. Phys. 328(1), 29-44 (2014)

50. Wang, W., Zhang, Z.: Blow-up of critical norms for the 3-d Navier-Stokes equations. Sci. China Math. 60(4), 637-650 (2017)

51. Weissler, F.B.: The Navier-Stokes initial value problem in $L^{p}$. Arch. Rational Mech. Anal. 74(3), 219230 (1980)

52. Yamazaki, M.: The Navier-Stokes equations in the weak- $L^{n}$ space with time-dependent external force. Math. Ann. 317(4), 635-675 (2000)

Communicated by K.Nakanishi 